\title{
Monitoring Occupancy and Office Equipment Energy \\ Consumption Using Real-Time Location System and \\ Wireless Energy Meters
}

\section{Nassim Masoudifar}

\author{
A Thesis \\ In the Department \\ Of \\ Building, Civil, and Environmental Engineering \\ Presented in Partial Fulfillment of the Requirements \\ For the Degree of Master of Applied Science (Building Engineering) at \\ Concordia University \\ Montreal, Quebec, Canada
}

August 2014

(C) Nassim Masoudifar 


\section{CONCORDIA UNIVERSITY}

\section{School of Graduate Studies}

This is to certify that the thesis prepared

By: $\quad$ Nassim Masoudifar

Entitled: $\quad$ Monitoring Occupancy and Office Equipment Energy

Consumption Using Real-Time Location System and Wireless Energy Meters

and submitted in partial fulfillment of the requirement for the degree of

Master of Applied Science (Building Engineering)

complies with the regulations of the University and meets with the accepted standards with respect to originality and quality.

Signed by the final examining committee:

\begin{tabular}{ll} 
Dr. R. Zmeureanu & Chair \\
Dr. Z. Zhu & Examiner \\
Dr. R. Zmeureanu & Examiner \\
\cline { 1 - 2 } Dr.A.Hammad & Supervisor \\
\hline
\end{tabular}

Approved by

Chair of Department or Graduate Program Director

Dean of Faculty

Date 


\section{ABSTRACT \\ Monitoring Occupancy and Office Equipment Energy Consumption Using Real-Time Location System and Wireless Energy Meters}

\section{Nassim Masoudifar}

Buildings are one of the major energy consumers because of the need to meet occupants' requirements. The commercial/institutional sector accounted for $14 \%$ of total energy consumption in Canada in 2009 while office buildings consumed 35\% of this amount. Auxiliary equipment used $19 \%$ of the total energy consumed in office buildings. Previous studies showed the impact of occupancy behavior on IT equipment energy consumption. This thesis proposes a new method for monitoring occupancy behavior and energy consumption of IT equipment. Two wireless sensor technologies are investigated to collect the required data and to build an occupancy behavior estimation profile: Ultra-Wideband Real-Time Location System for occupancy location monitoring and ZigBee wireless energy meters for monitoring the energy consumption of IT equipment. The occupancy monitoring data gained from the UWB are used to create the occupants' hourly profile. The occupancy profile based on short-time monitoring can be used to simulate long-term energy consumption. In conclusion, the comparison between the results shows up to $11 \%$ and $24 \%$ saving for heating loads and cooling loads, respectively. The proposed method profiles also resulted in up to $65 \%$ and $78 \%$ reduction for lighting and IT equipment energy consumption in the office, respectively. Therefore, dynamic occupancy driven profiles will reduce the energy consumption. 


\section{ACNOWLEDGEMENT}

I would like to express my sincere gratitude to my supervisor Prof. Hammad for the continuous support of my Master study and research, for his patience, motivation, enthusiasm, and immense knowledge. His guidance helped me in all the time of research and writing of this thesis.

Special thanks to my loved ones, my mother, my father, and my beloved sister for all the support they have provided me over the years. I will be grateful forever for your love. Words cannot express how indebted I am to all of the sacrifices that you've made. Your prayer for me was what sustained me thus far.

I would like to acknowledge the contributions of Mr. Dwahal Doshi for developing the python data logging program for the smart meters. I would like to appreciate the help my colleague Mandana to collect data and visualize the results. I would also like to give my special thanks to my colleagues Shide, Mohammad Mostafa, and Mohammad to participate in my tests without whom I could not accomplish my Master's degree.

At the end I would like express gratefulness to my friend Ali who was always my support in the moments when there was no one to answer my queries. 


\section{DEDICATION}

I would dedicate my thesis to my family with a special feeling of gratitude to my loving mum, Tahereh Rahmani, whose words of encouragement and push for tenacity ring in my ears and to my beloved sister, Negin who has never left my side and is very special to me. 


\section{TABLE OF CONTENTS}

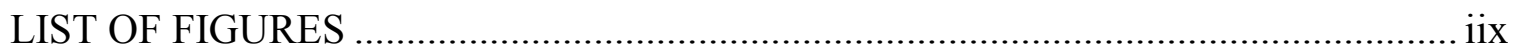

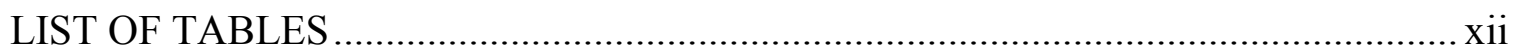

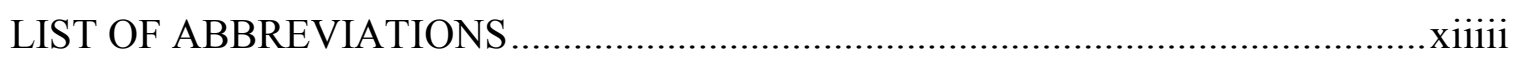

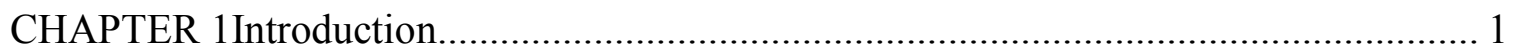

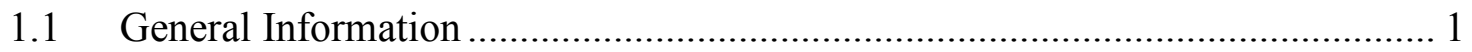

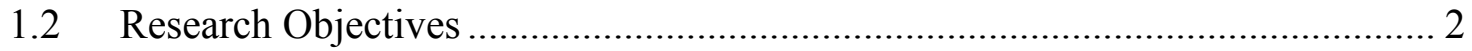

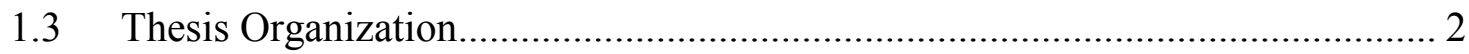

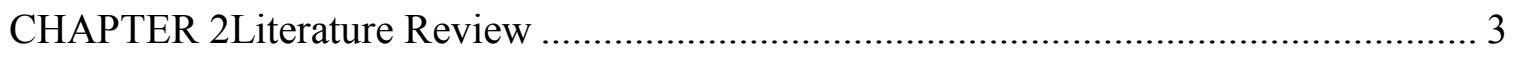

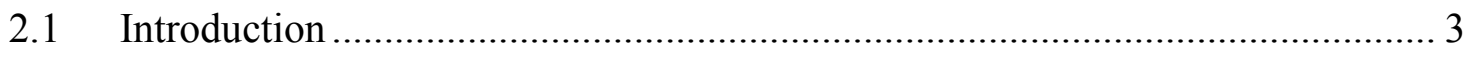

2.2 Building Energy Management Systems …………......................................... 5

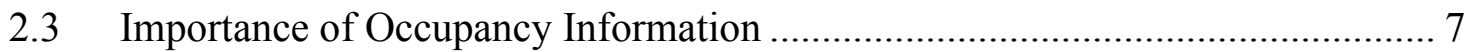

2.3.1 Statistical Model of Occupancy Profiles..................................................... 8

2.4 Localization and RTLS Technologies......................................................... 9

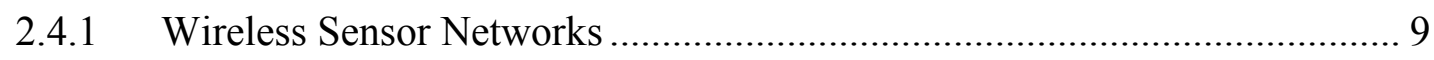

2.4.2 Real Time Location Systems .............................................................. 9

2.4.3 Indoor Localization Techniques and Systems ………………………........ 11

2.5 Energy Consumption Monitoring ................................................................... 13

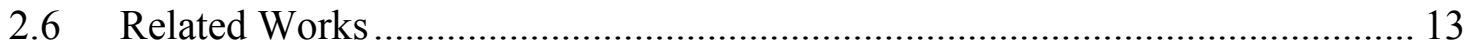

2.6.1 Using Tracking Technologies for Occupancy Monitoring ......................... 14

2.6.2 Simultaneous Monitoring of Occupancy and Energy Consumption/Control17

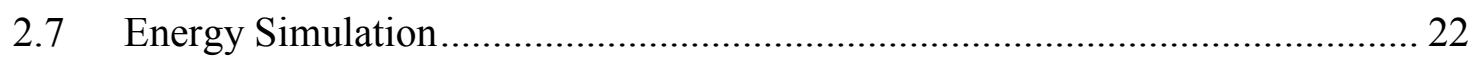

2.7.1 Simulation and Mea Dr. Z. Zhusurement of Energy Consumption ............ 22

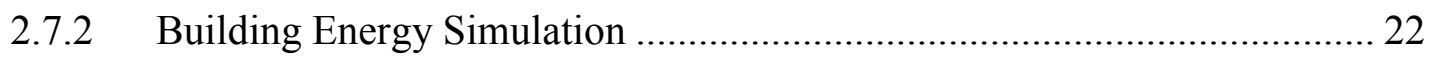

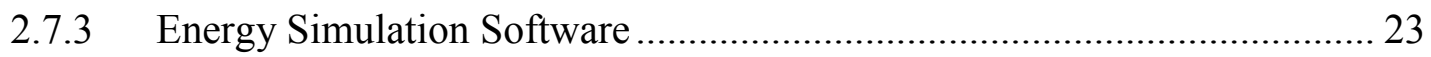

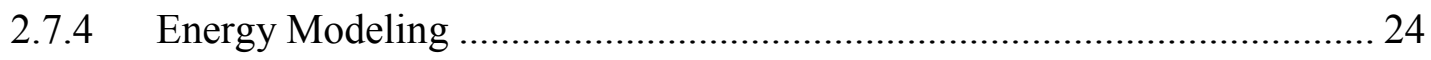

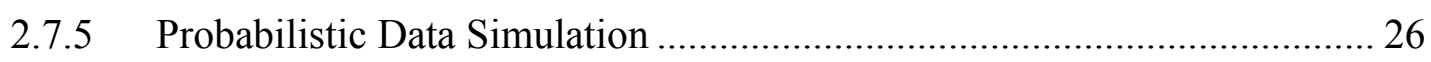

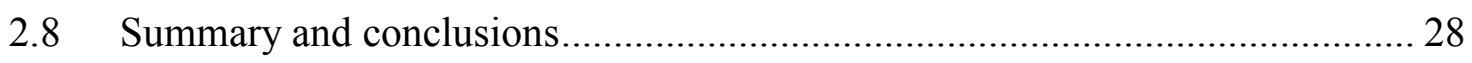

CHAPTER 3Requirements Analysis and Proposed Methodology ……………………... 29

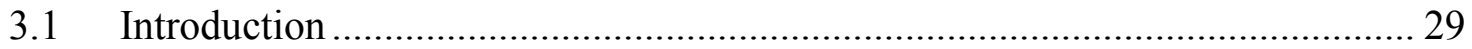

3.2 Technology Selection and Requirements Analysis ............................................ 30

3.2.1 UWB Technology for Occupancy Monitoring ......................................... 31 
3.2.2 ZigBee Technology and Requirements for Energy Monitoring ................. 34

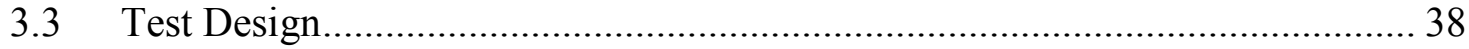

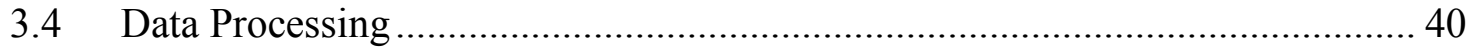

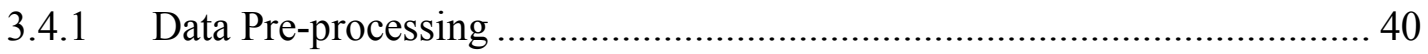

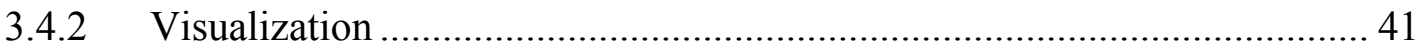

3.4.3 Statistical Occupancy Profiles .............................................................. 42

3.4.4 Data Fusion for Calculating Energy Waste ........................................... 44

3.5 Energy Consumption Simulation .......................................................... 45

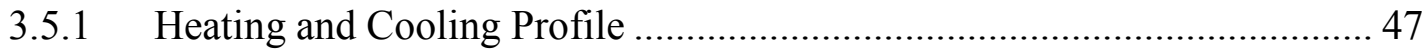

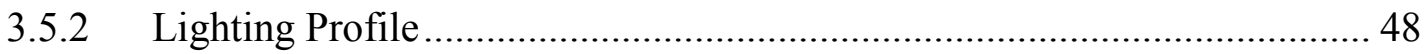

3.5.3 IT Equipment Profile ....................................................................... 50

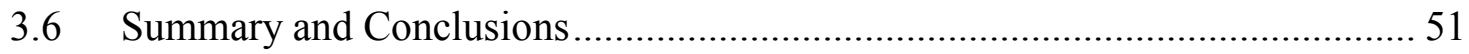

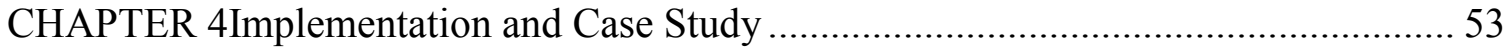

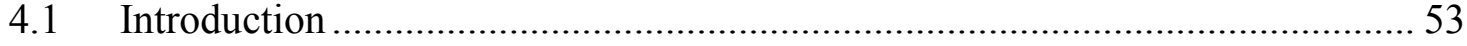

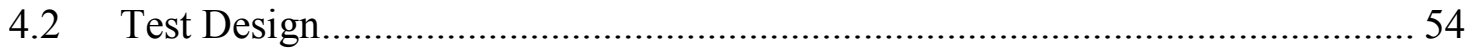

4.3 UWB and Energy Meters Raw Data .......................................................... 56

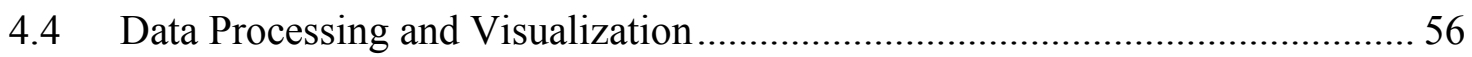

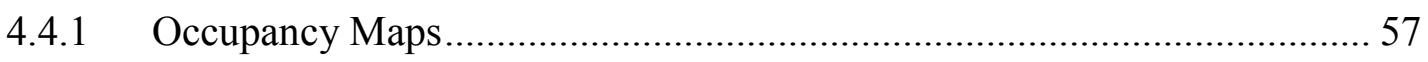

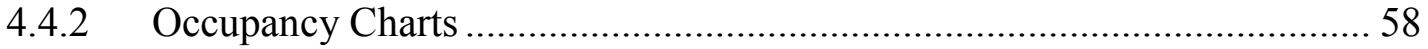

4.4.3 Zigbee Energy Meters Data Processing ..................................................... 60

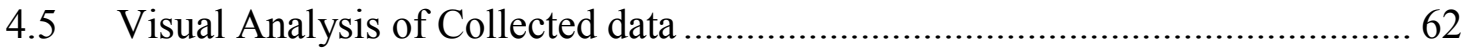

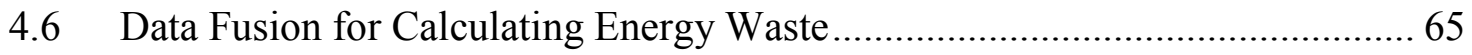

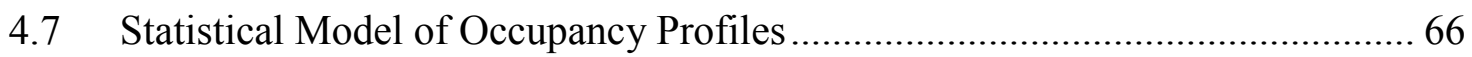

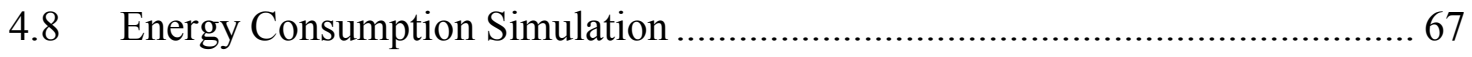

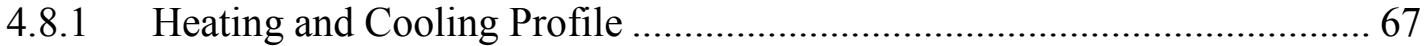

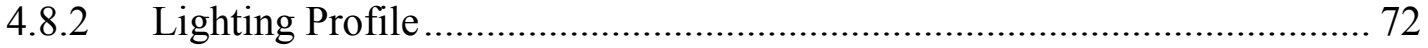

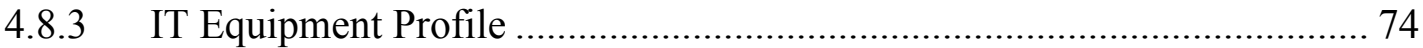

4.9 Energy Consumption Comparison between Proposed Profiles and Fixed Profiles80

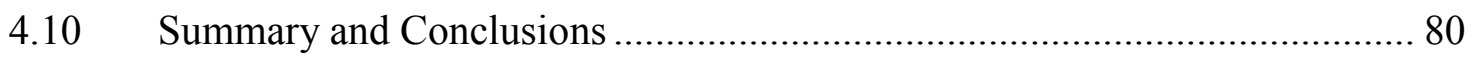

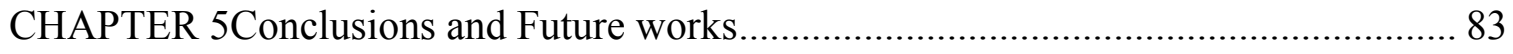

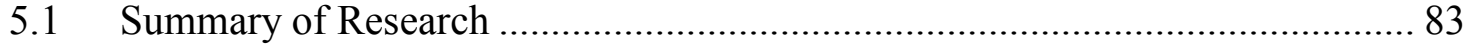

5.2 Research Contributions and Conclusions..................................................... 84

5.3 Limitations and Future Work ….............................................................. 86 


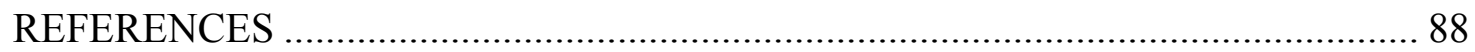

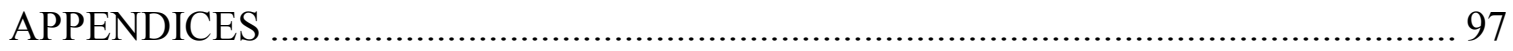

Appendix A- Ubisense Series 7000 Slim Tag Personnel or Assets............................ 97

Appendix B- ZigBee HA Wireless Metering Smart Plug Specifications .................. 100

Appendix C- Energy Simulation Configurations...................................................... 104

Appendix D- Smartplug Data Logging ............................................................. 106 


\section{LIST OF FIGURES}

Figure 2.1 Distribution of energy use in institutional/commercial buildings in Canada in 2009 (National Resource Canada, 2011). 4

Figure 2.2 Comparing Buildings energy consumption regarding occupants' behavior (Nguyen and Aiello 2013) 5

Figure 2.3 Interactions between humans and building components with impacts on heating and electricity demand (Wilke, et al. 2012) .................................................................... 8

Figure 2.4 Comparison of location technologies (Ward, 2007).................................. 11

Figure 2.5 Appliance power and energy consumption per user (Kim et al., 2008) ......... 19

Figure 2.6 Useful/wasted power and energy for TV (Kim et al., 2008) ......................... 19

Figure 2.7 Patterns of occupants vs. electricity consumption (Spataru et al., 2010) ........ 20

Figure 2.8 Schematic visualization of the zones considered in the test house (Spataru and Gillott, 2012) 21

Figure 3.1 Proposed method steps 30

Figure 3.2 (a) UWB sensor, (b) UWB slim tag, (c) UWB compact tag (Ubisense, 2013)

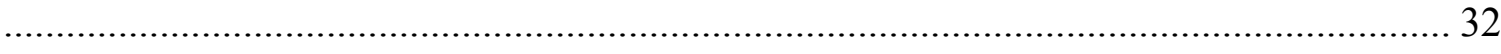

Figure 3.3 ZigBee network and products (ZigBee Alliance, 2013)............................. 35

Figure 3.4 (a) ZigBee gateway, (b) ZigBee energy meter (Smartenit, 2013) ................. 36

Figure 3.5 ZigBee network topology models (Ergen, 2004) ..................................... 38

Figure 3.6 Data sources (Masoudifar et al., 2014)...................................................... 39

Figure 3.7 Spatial temporal data visualization: (a) occupancy map, and (b) occupancy chart 42

Figure 3.8 Procedure to calculate the probabilistic individual occupancy profiles (Masoudifar et al., 2014) 44

Figure 3.9 Flowchart for calculating energy waste (Masoudifar, et al., 2014) 46

Figure 3.10 (a) Overall occupancy profile, (b) Occupant S weekdays probabilistic individual occupancy profile, (c) Occupant $\mathrm{S}$ weekend days probabilistic individual occupancy profile, (d) Heating and cooling simulation profile, (e) Occupant S weekdays IT equipment simulation profile, (f) Occupant S weekend days IT equipment simulation profile, (g) Lighting simulation profile 49

Figure 4.1 Layout of implementation of wireless sensor networks 55 


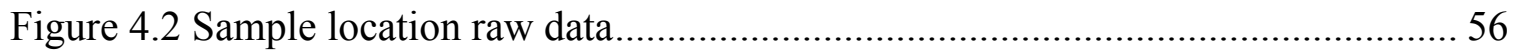

Figure 4.3 Sample ZigBee energy meters raw data ............................................... 56

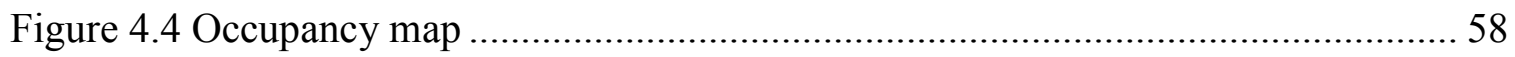

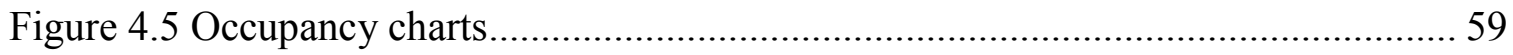

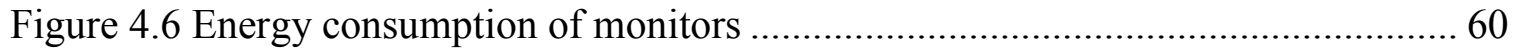

Figure 4.7 Monitors energy consumption varied regarding the percentage of brightness 62

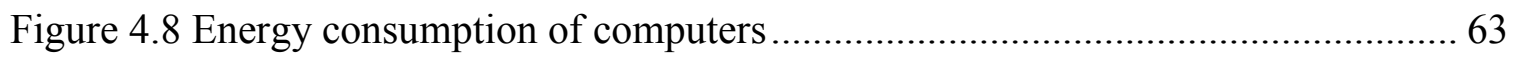

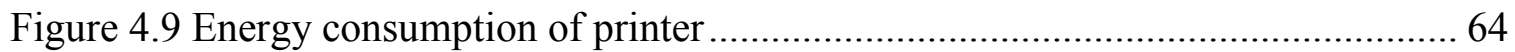

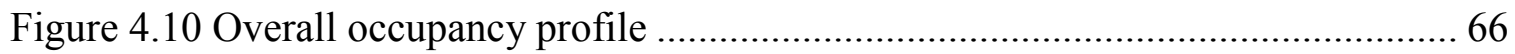

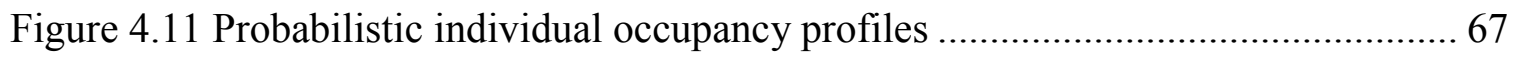

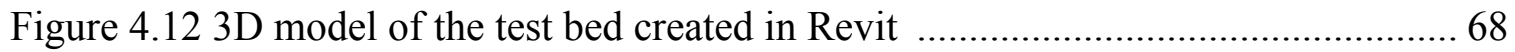

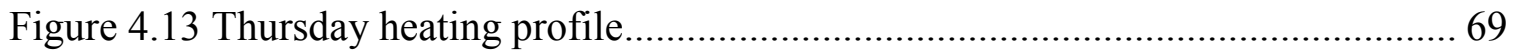

Figure 4.14 Weekly heating and cooling profile proposed based on monitored data....... 70

Figure 4.15 Annual heating and cooling loads based on proposed heating and cooling profile 71

Figure 4.16 Annual heating and cooling loads based on ASHRAE 6AM-10PM-no lunch71

Figure 4.17 Comparison between the energy consumption based on proposed heating and

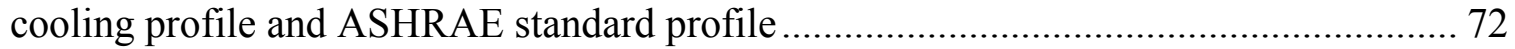

Figure 4.18 Weekly proposed lighting profile based on monitored data........................ 73

Figure 4.19 Comparison between the energy consumption based on proposed lighting profile and

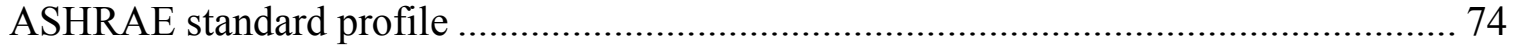

Figure 4.20 Occupant L weekly IT equipment energy consumption profile ................... 76

Figure 4.21 Comparison between the energy consumption based on proposed occupant L IT

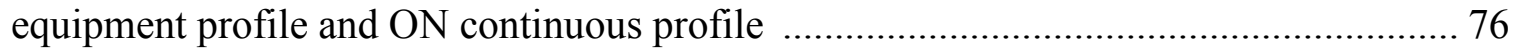

Figure 4.22 Occupant M weekly IT equipment energy consumption profile.................. 77

Figure 4.23 Comparison between the energy consumption based on proposed occupant M IT

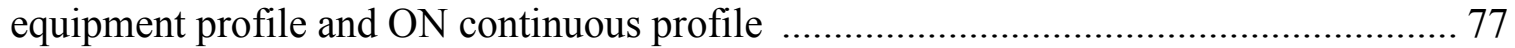

Figure 4.24 Occupant $\mathrm{N}$ weekly IT equipment energy consumption profile .................. 78

Figure 4.25 Comparison between the energy consumption based on proposed occupant N IT

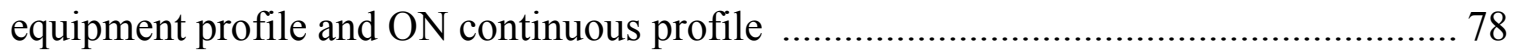

Figure 4.26 Occupant S weekly IT equipment energy consumption profile .................. 79 
Figure 4.27 Comparison between the energy consumption based on proposed occupant S IT equipment profile and $\mathrm{ON}$ continuous profile ............................................................ 79

Figure A.1 UWB slim tag component (Ubisense, 2013) .............................................. 97

Figure B.1 Smart plug components (Smartenit, 2013) ................................................. 100

Figure C.1 Selected profile for the office ................................................................. 104

Figure C.2 Heating system configuration .............................................................. 105

Figure C.3 Cooling system configuration ............................................................... 105 


\section{LIST OF TABLES}

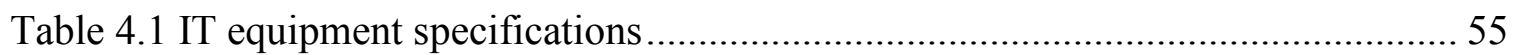

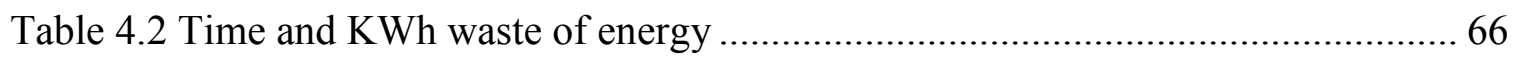

Table 4.3 Energy consumption comparison between proposed method profile and ASHRAE

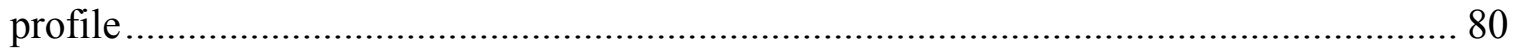

Table A.1 Slim tag specifications (Ubisense, 2013) …………………………………... 99

Table B.1 Smart plug specifications (Smartenit, 2013) ……………………………... 102

Table B.2 Status LED indication (Smartenit, 2013) ..................................................... 103 


\section{LIST OF ABBREVIATIONS}

2D

3D

$\mathrm{AC}$

A-GPS

ANN

AoA

ARIMA

BIM

CAD

$\mathrm{CO}_{2}$

GBS

gbXML

GPS

IFC

HAN

HVAC

$\mathrm{Hz}$

ID

IES $<$ VE $>$

IR

IT

KW
Two Dimensional

Three Dimensional

Alternating Current

Assisted Global Positioning System

Artificial Neural Networks

Angle of Arrival

Autoregressive Integrated Moving Average

Building Information Modeling

Computer-Aided Design

Carbon Dioxide

Green Building Studio

Green Building Extensible Markup Language

Global Positioning System

Industry Foundation Classes

Home Area Network

Heating, Ventilation, and Air Conditioning

Hertz

Identification

Integrated Environmental Solutions Virtual Environment

Infrared

Information technology

Kilo Watt 


\begin{tabular}{ll} 
KWh & Kilo Watt Hour \\
LAN & Local Area Network \\
L-HVAC & Lighting, Heating, Ventilation, and Air Conditioning \\
LCD & Liquid-Crystal Display \\
PC & personal computer \\
PoE & Power over Ethernet \\
PIR & Passive Infrared \\
RFID & Radio Frequency Identification \\
RSS & Received Signal Strength \\
RTLS & Real-Time Locating System \\
SCOPES & Smart Cameras Object Position Estimation system \\
TDoA & Time Difference of Arrival \\
ToA & Time of Arrival \\
TV & Television \\
USSU & User Simulation of Space Utilization \\
UWB & Ultra-Wide Band \\
V & Volt \\
VBA & Visual Basic for Application \\
Wi-Fi & Wireless Fidelity \\
WLAN & Wireless Local Area Network \\
WSN & Wireless Sensor Networks \\
\hline
\end{tabular}




\section{CHAPTER 1 Introduction}

\subsection{General Information}

Global climate change, a growing population, decreasing availability of fossil fuels, increasing environmental and economic concerns regarding energy use are driving forces towards more sustainable ways of responding to energy demands ( $\mathrm{Li} \&$ Becerik-Gerber, 2011). Energy consumption is widely increasing all around the world resulting in a global environmental problem. It is estimated that the world energy consumption will increase from 522 EJ in 2006 to $570 \mathrm{EJ}$ in 2015 and $730 \mathrm{EJ}$ in 2030. Due to the rising energy demand and diminishing energy resources, sustainability and energy conservation are becoming increasingly important topics (Conti et al., 2013).

Heating, cooling, lighting, and IT equipment energy consumption accounted for approximately $85 \%$ of the total energy consumption in the institutional/commercial buildings in Canada (National Resource Canada, 2011). Therefore, the demand for more accurate prediction of operational energy use is increasing in the institutional/commercial building sector. Occupancy information is an important variable which enables timely reaction to adjust energy load demands based on occupancy-driven management, drives a more optimized building operation schedule, and minimize energy consumption (Yang et al., 2012). In response to the occupancydriven system operations, different solutions have been proposed to predict occupancy patterns in energy consumption.

Since small power equipment, plug loads, and external lighting account for about $19 \%$ of the total energy consumption in an office building, the auxiliary equipment energy consumption 
should not be disregarded. Furthermore, small power loads can have a significant impact on the cooling loads of a building (Menezes et al., 2013).

\subsection{Research Objectives}

This research aims to achieve the following objectives: (1) to investigate the feasibility of two wireless sensor systems to collect the required data to better understand how occupancy behavior relates to energy consumption : Ultra-Wideband Real-Time Location System for occupancy location monitoring and ZigBee wireless energy meters for monitoring the energy consumption of IT equipment; (2) to create occupancy profiles based on the collected location data to optimise the energy simulation model; (3) to demonstrate the feasibility of the proposed method through the energy consumption simulation and calculating energy waste.

\subsection{Thesis Organization}

This thesis has been structured as follows: Chapter 1 gives an overview of the research topic and outlines sub-categories within the research undertaken. The scope and objectives of the proposed method are also stated. Chapter 2 reviews the existing literature on building energy consumption and the significant work that has already been done in the field of occupancy detection, occupancy behavior monitoring, and appliances energy consumption monitoring. In Chapter 3, the proposed method for real-time occupancy monitoring, measuring IT equipment energy consumption, data analysis demonstrating how the research method is applied to the collected data, and simulation are discussed in detail. The framework of the implementation, case study, findings of the proposed method, and analysis are presented in Chapter 4. Chapter 5 concludes this thesis with a summary of findings and outlines the potential future work. 


\section{CHAPTER 2 Literature Review}

\subsection{Introduction}

Buildings are one of the major energy consumers in Canada. In the commercial/institutional sector, space heating, cooling, and lighting systems were responsible for $64 \%$ of the total energy consumption of this sector in 2009, which means that they are prime targets for energy consumption optimization (National Resource Canada, 2011). Current facility management practices usually rely on gross generalizations to operate Heating, Ventilation, and Air Conditioning (HVAC), lighting and IT equipment systems, such as operating for the peak occupancy load, leading to more energy consumption than needed (Li \& Becerik-Gerber, 2011). These systems are based on standard set points, which are assumed to provide functionality and satisfaction for the majority of the occupants. These set points are defined under controlled environmental conditions and use assumptions about buildings and occupancy behavior (Jazizadeha et al., 2012). ASHRAE Standard 55 for instance, defines the comfort range for typical occupants at various activities and environments (Hong et al., 2013).

Previous research suggested a variety of modifications in the performance of existing buildings in order to minimize their energy use, which will result in a significant energy consumption reduction in office buildings during a long-term (Nguyen \& Aiello, 2013). Figure 2.1 shows the average distribution of energy consumers in commercial/institutional buildings in Canada. Regarding the increasing demand for more realistic prediction of operational energy use in the institutional/commercial building sector, auxiliary equipment should not be disregarded since it typically accounted for $19 \%$ of total energy used in this sector. In an office building, unregulated loads (including all small power equipment and plug loads, as well as external lighting) typically 
represent a large proportion of the total energy consumption. Furthermore, small power loads can have a significant impact on the cooling loads of a building (Menezes et al., 2013).

Occupancy information is an important variable which enables timely reaction to adjust energy load demands, drive a more optimized building operation schedule, and minimize energy consumption (Yang, 2012).

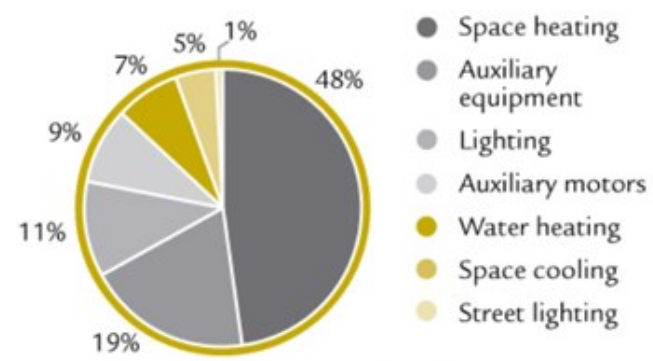

Figure 2.1 Distribution of energy use in institutional/commercial buildings in Canada in 2009 (National Resource Canada, 2011)

Occupancy behavior refers to an occupant's movement, personal habits, and responses to his/her needs by interacting with the building systems which have direct and decisive impacts on the energy performance of buildings (Nguyen and Aiello, 2013). Results from studies and implementation by academia and industry promised saving in the range of $2.7-55 \%$ in energy consumption by considering occupancy behavior. A number of academic studies have been done in order to test a variety of occupancy and energy consumption monitoring systems to collect real-time data and to create user profiles (Ueno et al., 2006; Petersen et al., 2007; Fischer, 2008; Allcott, 2010; Faruqui et al., 2010; Peschiera and Taylor, 2012; Vassileva et al., 2012).

The topic of occupancy-driven management has attracted considerable attention in academic research due to the potential energy savings. To lay the basis of occupancy-driven system operations, different solutions have been proposed to monitor the occupants in a building. 
Simulation results showed that occupancy-based control can result in $10-60 \%$ in energy saving (Tachwali et al., 2007; Erickson et al., 2010; Sun et al., 2011; Li and Becerik-Gerber, 2011). However, these solutions have certain limitations in terms of their accuracy and intrusiveness.

Nguyen and Aiello (2013) proposed that occupancy presence and behavior in buildings have large impacts especially on space heating, cooling and ventilation demand, lighting and appliances energy consumption, and building controls. Figure 2.2 demonstrates that building occupants with careless behavior can add one-third to a building's designed energy performance, while occupants with conservation behavior can save a third.

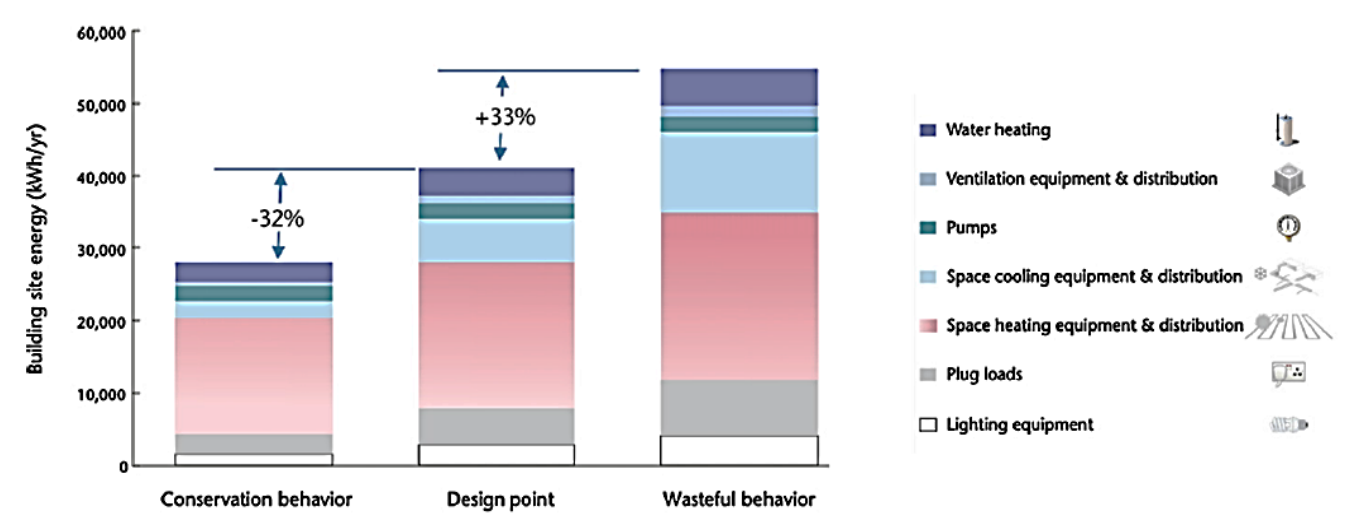

Figure 2.2 Comparing Buildings energy consumption regarding occupancy behavior (Nguyen and Aiello, 2013)

More importantly, predicted occupancy profiles play a significant role in the performance of the building. Thus, much research has been focused on predicting occupancy profiles for energy consumption (Nguyen and Aiello, 2013).

\subsection{Building Energy Management Systems}

Many research efforts have been focused on sustainable practices to reduce energy consumption in buildings. The general vision for a sustainable building is an intelligent building, which is responsive to the requirements of the occupants, environment and society by being functional 
and productive for occupants, and sustainable in terms of energy consumption and $\mathrm{CO}_{2}$ emissions. To achieve this level of performance for the buildings, the interaction between building systems, processes and occupants should be considered. This interaction requires monitoring and control in the buildings by using the obtained information for improving the performance and quality of new and existing buildings. The term 'intelligent buildings' refers to buildings equipped with technology that allows monitoring of their occupants and facilities to automate and optimize the control of appliances, in particular, lights, HVAC system, and home appliances, with the goal of saving energy (Croome, 2011).

A common way to model an intelligent building environment control system is to divide it in four cooperating layers. The physical layer contains the hardware within the building including individual devices, transducers, and network hardware, and is in charge of collecting data from the environment and performing actions. The communication layer allows the exchange of information within the network of devices. The information layer gathers, stores, and generates knowledge useful for the decision layer (Nguyen and Aiello, 2013).

Building automation generally refers to the deployment of computer-based control system to monitor and control the physical parameters of the environment, security access, or fire alarm systems in a building. The intelligent building management system aims to keep temperature and air quality within a specified range, control lighting, monitor performance of all systems, and send out alarm signals to maintenance personnel when any fault occurs. The general objective of a building energy management system is to fulfill the occupancy comfort needs while reducing energy consumption during building operations. The energy demand of buildings is related significantly to the indoor environment and building operations scenario. By using a wireless sensor network (WSN) to measure the physical parameters of the environment and then process 
the data, a dynamic picture of the actual state of the indoor environment can be produced. This can feed the Facility Management systems and lead to the increased well-being of occupants as well as decreased energy consumption. The increasing miniaturization of radio frequency devices and micro-electromechanical systems, as well as the advances in wireless technology, have generated a great interest in WSNs due to the fact that they can provide an infrastructure for gathering information about the physical world, including equipment and the behavior of people (Nguyen and Aiello, 2013).

\subsection{Importance of Occupancy Information}

Climate, building envelope, building equipment (energy and water services systems), operation and maintenance, occupancy behavior, and indoor environmental conditions are six major factors that determine the energy performance of buildings. Among these six factors, the occupancy behavior is the least studied and is simplified during the building's design and operation. Occupancy behavior refers to an occupant's movement, personal habits, and responses to his/her needs by interacting with the building systems (how often and for how long) which has direct and decisive impacts on energy performance of buildings. The occupancy behavior varies with time, space and individual (Hong et al., 2013).

Figure 2.3 summarizes interactions between human beings and building components. The use of many electrical appliances results in considerable impacts on electricity demand and involves heat gains which have important influences on heating and cooling demand. Occupancy presence and behavior in buildings have been shown to have large impacts on space heating, cooling and ventilation demand, energy consumption of lighting and space appliances, and building controls (Wilke et al., 2012). 


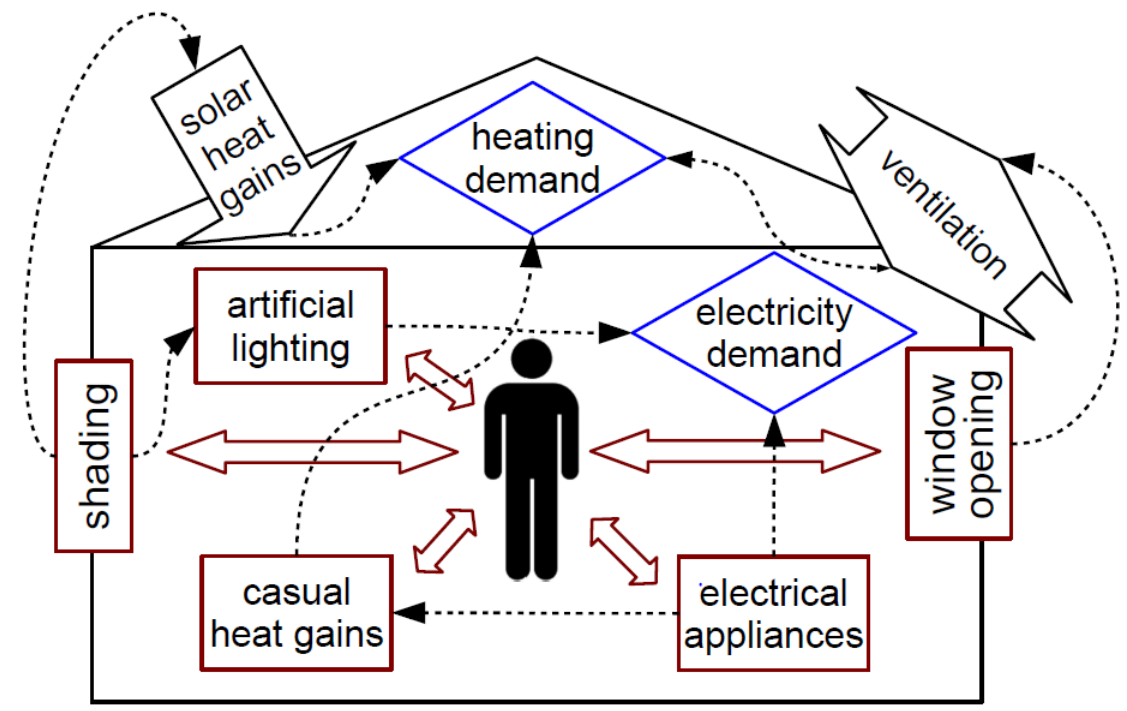

Figure 2.3 Interactions between humans and building components with impacts on heating and electricity demand (Wilke et al., 2012)

Real-time occupancy information has long been used for the control of various devices like artificial light, HVAC devices, IT equipment, and so on (Page et al., 2008).

\subsubsection{Statistical Model of Occupancy Profiles}

In an office building, usually there is a typical movement pattern for the occupants during week days and weekend days. Office occupants usually come to the office in the morning, they may have a lunch break around noon, and getting off work in the evening or night (possibly with overtime), which leads to the variation of the number of occupants of a typical office building during the working periods. Moreover, the occupancy for each zone of the office building would change since the occupants walk around among the spaces inside and outside of the office for a variety of reasons. The frequencies of occupants leaving their offices and the corresponding durations of absences have significant impacts on energy use and the operational controls of buildings (Hong et al., 2013).

Indoor environmental parameters and occupant's interactions with IT office equipment have impacts on energy consumption which are measured and analyzed to identify occupancy 
behavior profile and develop mathematical probabilistic models. Occupancy profile is one of the driving factors behind discrepancies between the measured and simulated-designed energy consumption of buildings. Therefore, optimizing the occupancy behavior can result in minimizing building energy consumption and waste of energy (Hong et al., 2013).

Hong et al. (2013) used statistical methods to analyze the occupancy status, based on measured lighting-switch data in five-minute intervals, for a total of 200 open-plan offices. Five typical occupancy profiles were identified based on the average daily 24-hour profiles of the presence of occupants in their offices.

\subsection{Localization and RTLS Technologies}

\subsubsection{Wireless Sensor Networks}

A sensor network is an infrastructure comprised of sensing (measuring), computing, and communication elements that gives an administrator the ability to instrument, observe, and react to events and phenomena in a specified environment. There are four basic components in a sensor network: (1) an assembly of distributed or localized sensors; (2) an interconnecting network (wired or wireless-based); (3) a central point of information clustering; and (4) a set of computing resources at the central point to handle data correlation, event trending, status querying, and data mining (Sohraby et al., 2007).

\subsubsection{Real Time Location Systems}

Although there has been considerable effort to support the dynamic stochastic modelling of occupancy information, activities and the use of electrical appliances, there is no existing approach which accurately accounts for significant variations between individuals. Either the calibration procedures are designed to match average behaviors, or the level of detail is not tested 
for statistical significance of parameters (Page et al., 2008). Real-Time Location Systems (RTLSs) are used to automatically identify and track the location of objects or people in real time, usually within a building or other contained areas. An RTLS is a wireless system with the ability to locate the position of an item anywhere in a defined space at a point in time that is, or is close to, real time. RTLS allows determining the location of assets or people at a specific time and frequency of update. Different technologies can be used in an RTLS such as sound, ultrasound, Bluetooth, Wi-Fi, RFID, ZigBee, UWB, GPS, cellular phone, camera vision, infrared and light, among others (Malik, 2009). Location technologies estimate a location of a point in two-dimensional (2D) or three-dimensional (3D) space in relation to a coordinate system where some references are known. Distance and observable angles are in most of the cases the base for those findings and can be obtained from different sources such as arrival times, arrival time differences, and field strength, among others. The main objective of a location system is to determine the exact position of people or assets by minimizing the errors in the measurements. By knowing the relative location of an object in relation to sufficient reference points, the absolute location of the object can be determined (Ward and Webster, 2009).

Wireless RTLS tags are attached to objects or worn by people to be tracked or managed. In most RTLSs, fixed reference points receive wireless signals from tags to determine their location. RTLSs are generally used in indoor and/or confined areas, such as buildings.

Each location system has its intrinsic and cost limitations. Recently, location technologies are focused on improving the accuracy of the position of one system and data fusion of different systems. To get the maximum benefit of these technologies, different aspects must be taken into account when deciding which technology must be used since not every technology is suitable for each application. The accuracy, advantages and limitations of the RTLS directly affect the final 
purpose of the location system. Therefore, the requirements of the location system contribute to the accomplishment of the final goal of the RTLSs. Appropriate applications result in achieving adequate equilibrium between system requirements, technological advantages, and associated costs (Munoz et al., 2009).

Figure 2.4 demonstrates the comparison between different location technologies, such as passive RFID, electromagnetic, laser, ultrasound, infrared (IR) proximity, conventional Radio Frequency (RF) timing, UWB, Wireless Local Area Network (WLAN), Received Signal Strength (RSS), and Assisted GPS (A-GPS). This comparison is done based on the accuracy and the coverage offered from each technology and identifying the ideal as the technology that can achieve accuracy less than $0.3 \mathrm{~m}$ and with coverage more than $100 \mathrm{~m}$ (Ward, 2007).

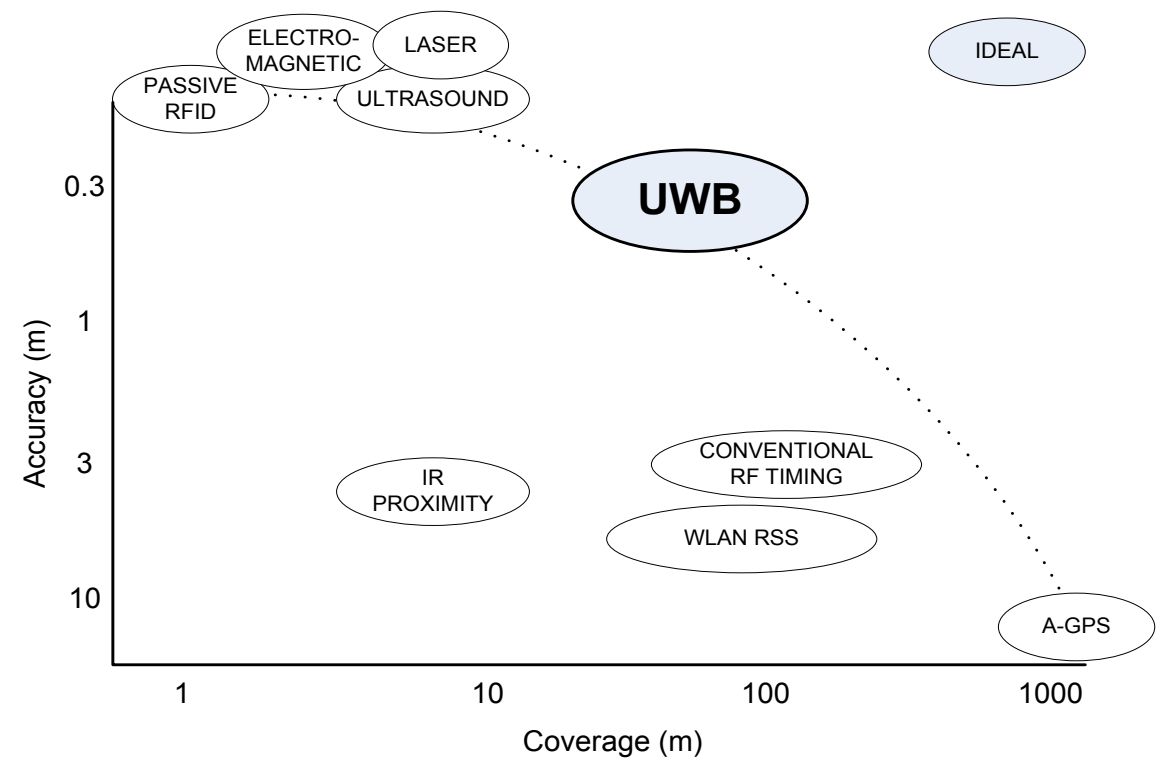

Figure 2.4 Comparison of location technologies (Ward, 2007)

\subsubsection{Indoor Localization Techniques and Systems}

Localization is the technique to determine the position of people or assets. The position information enables location-based protocols to navigate, track or monitor a person or an asset 
(Sana, 2013). Localization methods for identifying locations in both indoor and outdoor environments cannot be the same because: (1) Indoor environments are more complex; (2) There are signal interferences and reflections inside building; (3) It is highly dependent on the environment such as the position of objects or the behavior of persons; (4) Indoor communication link is unreliable. Many location based protocols and services are proposed for outdoor and indoor environments. For outdoor environments, we can use fixed sensors or GPS based sensors. GPS is the most widely used satellite based positioning system, which offers maximum coverage. GPS cannot be deployed inside buildings since it requires line-of-sight transmission between receivers and satellites which is not possible in indoor environments. Many options are available for the design of RTLSs in indoor environments such as infrared (IR), ultrasound, radio-frequency identification (RFID), wireless local area network (WLAN), Bluetooth, ultra-wideband (UWB), magnetic signals, vision analysis and audible sound, light, and so on. Depending on the priority of the user, different positioning systems are developed (Levine et al., 2007; Wong et al., 2005)

The design and deployment of a system for obtaining location and spatial information in an indoor environment is a challenging task for several reasons including user privacy, management overheads, systems scalability and harsh nature of indoor wireless channel (i.e. metal reflection, interference with noise). Recently, indoor localization systems have been designed to provide location information of persons and devices in the indoor environments (Aggarwal and Ryoo, 2011). 


\subsection{Energy Consumption Monitoring}

Smart metering in buildings is one of the methods to measure the energy consumption which helps people understand the influence of occupancy behavior on energy consumption levels. Experimental data from metering can provide people the ways in which they can change their behavior and reduce the energy consumption levels and become more sustainable. Meters can be installed to monitor instantaneous energy consumption and allow a control system to vary lighting and HVAC in response to loads, changes in weather, and spot price of energy. Smart meters enable facilities managers to identify areas that require attention (Jazizadeha et al., 2012).

\subsection{Related Works}

A number of academic research projects have been done in order to test a variety of occupancy and energy consumption monitoring systems to evaluate the impact of occupancy behavior on IT equipment energy consumption and achieve real-time data to create user profiling. As summary of the related works is provided below. WSNs are the common approach of the various projects to address user activity recognition. Furthermore, most of the projects stress the requirement of not resorting to any advanced sensors, such as cameras, which are expensive and generate privacy issues. Instead, simple, wireless, binary sensors were favored since they were cheap, easy to retrofit in existing buildings, and require minimal maintenance and supervision. Simple sensors were used in many intelligent buildings in the interest of activity recognition. 


\subsubsection{Using Tracking Technologies for Occupancy Monitoring}

\subsubsection{Passive Infrared (PIR) Motion Sesnsors, Bluetooth, and Carbon-Dioxide Sensor}

Delaney et al. (2009) introduced LIGHTting evaluation through wireless sensors (LightWiSe). LightWiSe used two common sensing devices: (1) a light detector used for detecting ambient light and luminaries state, and (2) PIR sensor to detect people presence. Aggarwal and Ryoo (2011) utilized a deployment of PIR and door sensors to obtain a binary indication of occupancy (presence/ absence). In order to demonstrate the benefits of the presence system, they simulated an example building along with its HVAC energy consumption.

Newsham and Birt (2010) developed an Autoregressive Integrated Moving Average (ARIMA) model to forecast the power demand of the building in which a measure of building occupancy is a significant independent variable and increases the model accuracy. To gather data related to total building occupancy, contact closure sensors were placed on various doors, and PIR motion sensors and a carbon-dioxide sensor were positioned in a circulation area. In addition, they collected data on the number of people who had logged into the network on each day, network activity, electrical energy use (total building, and chilling plant only), and outdoor temperature of a three-story building in eastern Ontario, Canada, comprising laboratories and 81 individual work spaces. Padmanabh et al. (2009) studied the iSense system to recognize two states of a conference room (meeting state and no meeting state) by using a network of wireless microphone, PIR, light, and temperature sensors. iSense was able to save $13 \%$ of air conditioning and lighting electricity by the alerting mechanism in place. 
Lee and Mase (2002) used wearable motion sensors to detect the location and movements of a person. The person motions were measured through the rate of change in the person's displacement from the initial start position.

One of the common approaches was using a variety of simple sensors and applying Bayesian inference techniques to infer the state of user(s) in the environment and hence adapt the power states of associated devices. For example, Harris and Cahill (2005) examined the power management of users' stationary personal computers (PCs) in an office environment. The solution used two simple location-aware policies based on the locations derived from detecting users' Bluetooth-enabled mobile phones, and tried to dynamically and probabilistically assign each of the users' state (using, about to use, not using) in order to set the power state of the associated PC.

Harle and Hopper (2008) installed a personnel tracking system at the ATandT Research building in Cambridge, UK, which featured an ultrasonic location system that provided three dimensional tracking. They explored the use of location-awareness to dynamically optimize the energy consumption of an office. They used a large corpus of captured location data from a nonacademic environment with 50 rooms, involving around 40 people with various roles, captured during 60 working days randomly selected from a year. They analyzed the collected location data to form a picture of how people work and what energy savings might reasonably be expected if it was possible to prevent device 'idling'. They used coarse location in the form of proximity detection using Bluetooth, microphones to detect activity, and webcams and facial tracking to estimate when a display was being observed. They characterized users as HeavyUse ( $85 \%$ of their time using their computer when in its vicinity) and LightUse (significantly less time spent using their PC when in its vicinity). 


\subsubsection{Video Processing}

There are other methods for detecting occupancy including using camera-based systems which also may bring up concerns relating to cost and privacy issues. Erickson et al. (2009) carried out a research based on occupancy measurement, modelling and prediction for building energy savings. They developed a system that tracks users' movements in building spaces using a camera network solution called a distributed Smart Cameras Object Position Estimation system (SCOPES).

Kamthe et al. (2009) deployed a wireless camera sensor network for collecting data regarding occupancy in a large multi-function building. Using the data collected from this system, they constructed multivariate Gaussian and agent-based models for predicting user mobility patterns in buildings.

\subsubsection{UWB and RFID Localization}

Many studies provided an occupancy detection solution that used Radio Frequency Identification (RFID) sensors as an effective technology for indoor localization. Li et al. (2012) proposed a system built on RFID technology which could provide not only the rate of accurate sensing of occupants' presence (e.g., occupied or unoccupied), but also detect the number of occupants. Occupancy detection rates were provided for multiple occupants performing multiple activities (i.e., walking, seated, and standing).

Liu et al. (2007) analyzed systems for indoor localization based on radio-based techniques and compared them with a metric that evaluated their positioning precision, complexity, robustness and cost. It was determined that systems using UWB technology have the highest precision and 
accuracy. Gillott et al. (2006) used an active RFID tag system investigating the relationships between occupancy and space use in a domestic environment.

Such applications were extended and included sensors socket meters to detect when energy was consumed and then this information was added to the motion information and associated with the activity location through automated software so that the interaction between occupants and energy was determined and visualized in real time (Liu et al., 2007).

\subsubsection{Simultaneous Monitoring of Occupancy and Energy Consumption/Control}

Hagras et al. (2004) used temporal information in behavior recognition. They developed iDorm as an Embedded Agent which was able to recognize three activities of a person, namely, sleeping, working, and entertaining. The system was also able to learn users' preferences, to predict users' needs (e.g., light intensity, temperature), and to self-adjust the system behavior (including lighting, heating, and cooling) when users change their habits.

Marchiori and Han (2010) proposed a general framework where building systems could share information in order to optimize performance. Their prototype was deployed in two graduate student offices on their campus, to monitor occupancy information and several switched devices including two Liquid-Crystal Displays (LCDs), a laser printer, two powered speakers, a desk lamp, a microwave, and a coffee pot. A real test-bed was used to evaluate the energy saving

potential of an occupancy-based control strategy for plug loads. Harris and Cahill (2005) and Nguyen and Aiello (2013) tried to automatically recognize typical activities of office presence and used them (five typical activities, namely, working with/without PC, having a meeting, presence, absence) as drivers to control the lighting system and plug loads to save energy. 
Kim et al. (2008) proposed SPOTLIGHT, a prototype based on previous studies showing the fact that better understanding of an individual's energy consumption helps people to lower their energy footprint significantly. SPOTLIGHT profiled an individual's natural resource consumption pattern in real time using wireless sensor network technology in the area of home environment. The SPOTLIGHT prototype consisted of power measurement, activity monitoring and energy resource management subsystems providing a personalized report on resource consumption at an appropriate spatiotemporal resolution to each of the individuals in a household in order to induce resource-conserving personal behavioral changes.

The proposed system was designed based on fusing several different networked embedded sensing systems, and machine learning techniques and algorithms in order to provide accurate resource consumption estimation. Their prototype showed that with measuring the plug loads energy consumption, it is possible to identify certain user activity. These measurements were used to attribute energy consumption to specific users. Additionally, the system showed that users can identify where they use most of the energy, and where they waste a lot of energy. The identified activities were watching television (TV), using coffee maker, and using living lamp/bedroom lamp. Figure 2.5 shows the power consumption of the users for the different instrumented appliances and the total energy consumption in this study. In Figure 2.5(b), it is shown that, for example, only user 201 used the coffee maker. So no data was collected for user 200. The graph in Figure 2.5(e) shows the total power consumption.

Figure 2.6 highlights the amount of useful and wasted energy consumed by the TV. The energy consumed was useful if one of the users was within the proximity and hence benefiting from the consumption. The energy was wasted in case that there was no user in the appliances vicinity (Kim et al., 2008). 

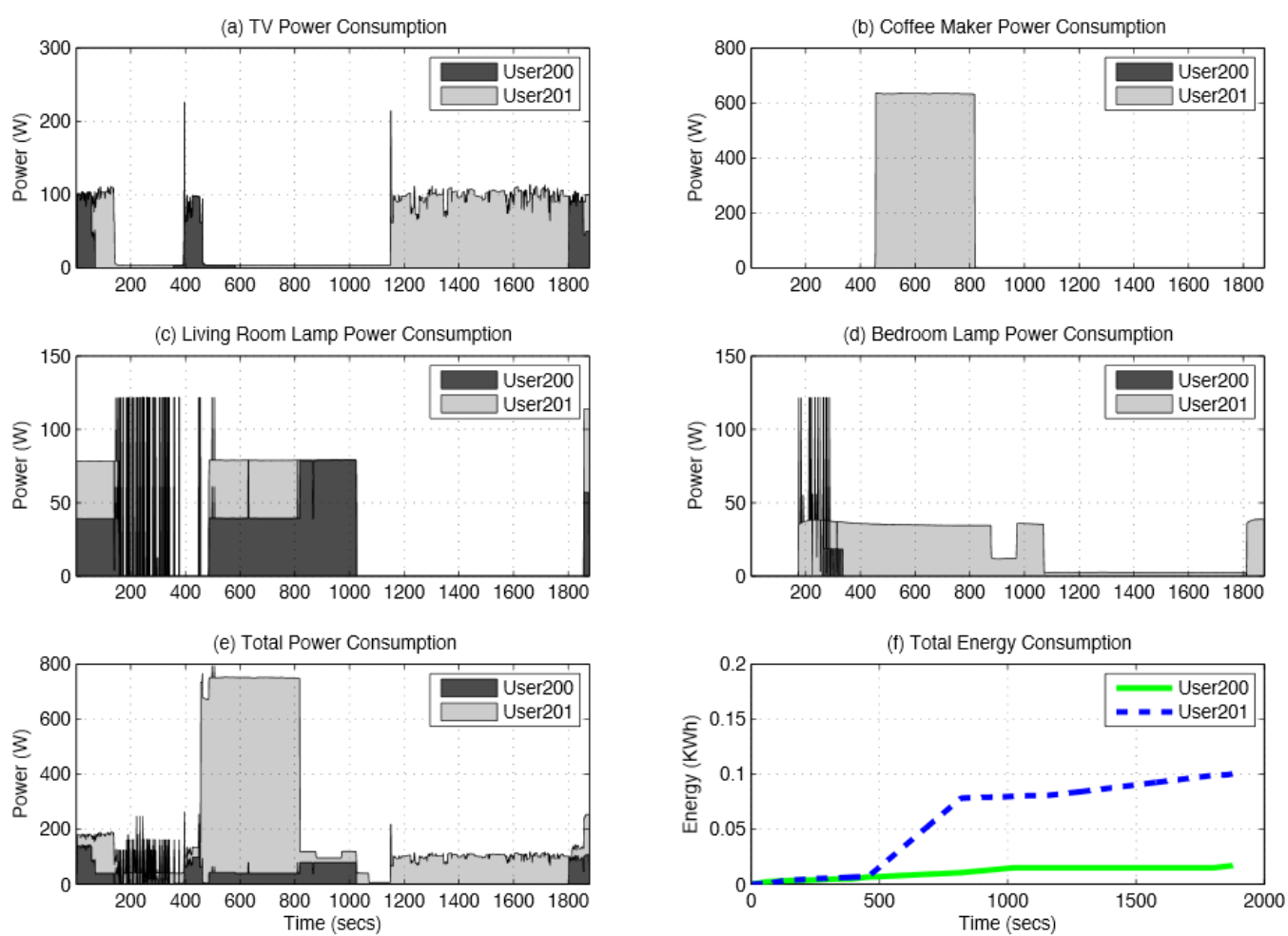

Figure 2.5 Appliance power and energy consumption per user (Kim et al., 2008)
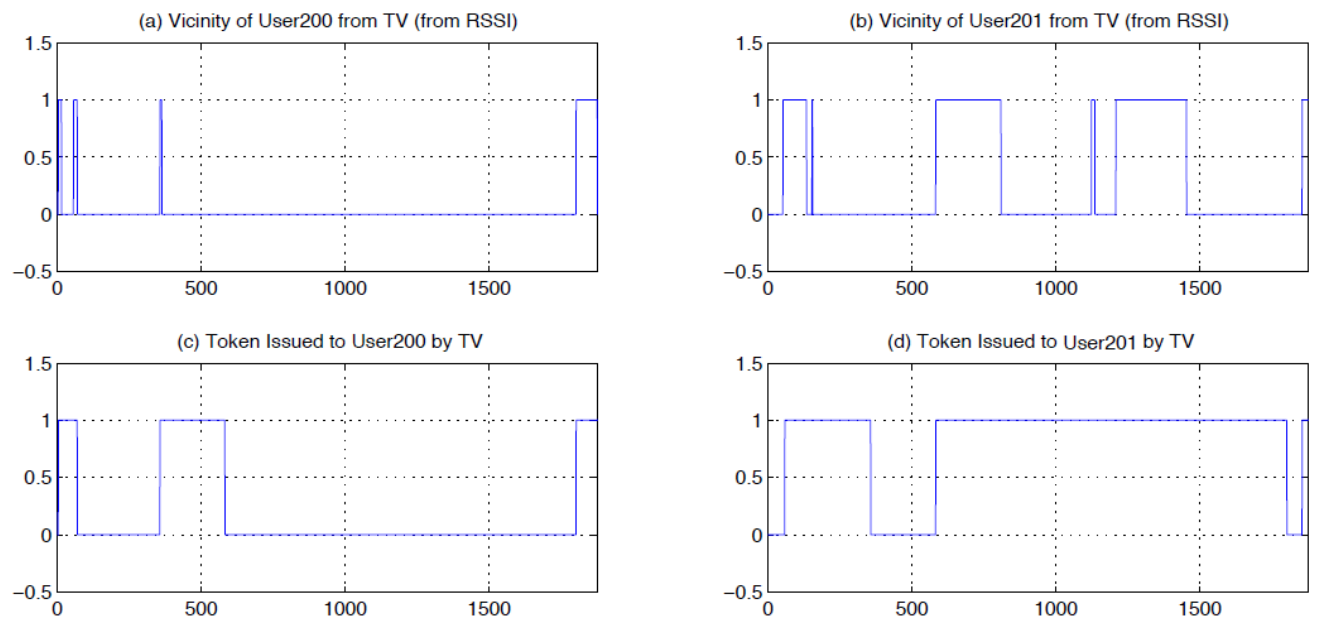

(e) Power Consumed by User200 for TV
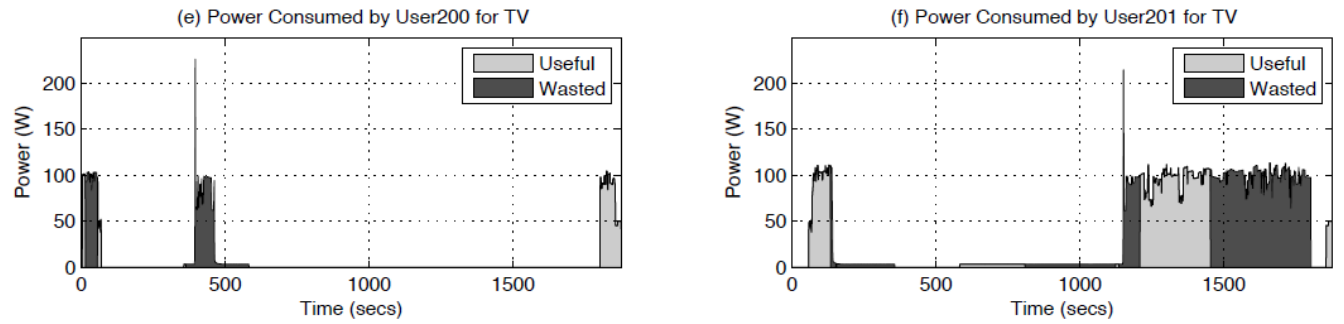

Figure 2.6 Useful/wasted power and energy for TV (Kim et al., 2008) 
Spataru et al. (2010), monitored a house as a test bed in order to collect occupants' real-time energy usage and location data. The occupancy patterns and space use were recorded using RTLS UWB technology to track the patterns of space usage in the house for time and location which are shown in Figure 2.7. Electricity consumption was monitored using whole house, circuit and appliance meters (including lighting). The tracking device consisted of a network system of fixed sensors positioned around the house to pick up the 3D location of compact tags which were carried by the occupants.

In another research, Spataru and Gillott (2012) monitored and analyzed occupancy activities and equipment energy consumption in a test house. The house was occupied by a family on the University of Nottingham campus. They used an RTLS-based UWB system for real-time localisation and identification of occupants. They also measured and monitored the electricity energy consumption in the hosue using whole house circuit and appliance meters.

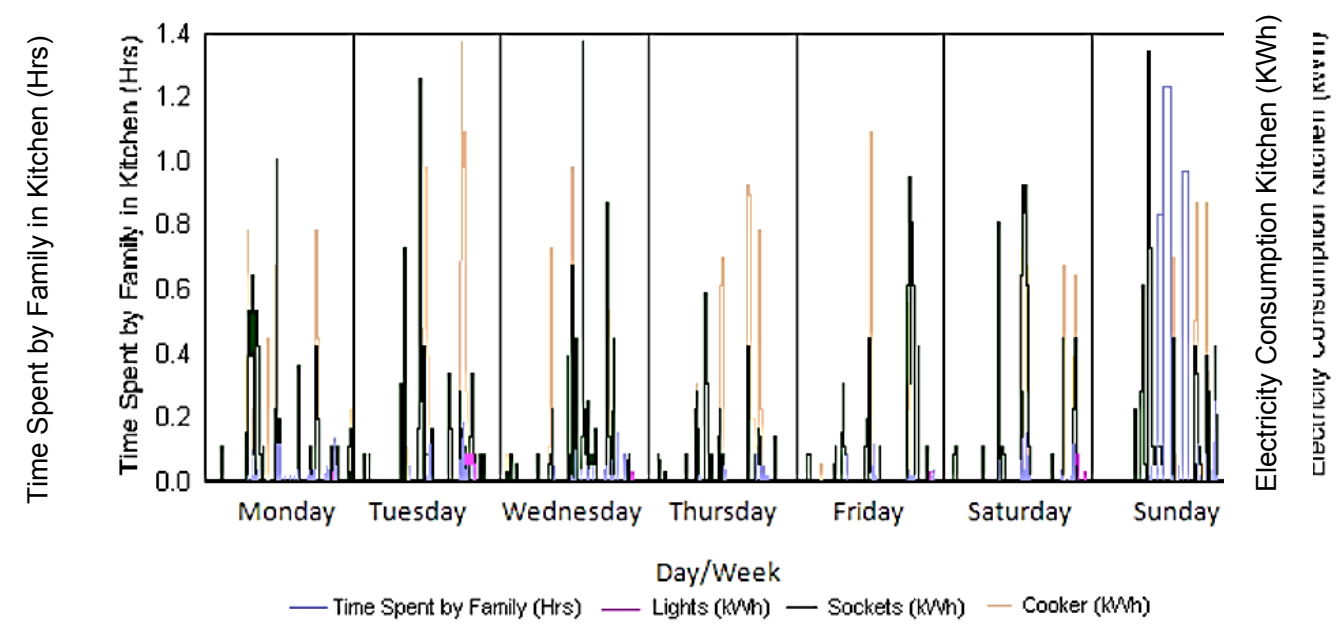

Figure 2.7 Patterns of occupants vs. electricity consumption (Spataru et al., 2010)

Occupants' locations were tracked and identified to monitor their activities at different times to predict energy usage in the house. The location data were interlinked with energy consumption and activity information based on sensor data in a fully monitored house to predict energy 
consumption precisely. However, the disadvantage of using this system was the high cost of the system and the fact that occupants have to wear the tags.

As represented in Figure 2.8, the energy home test-bed consisted of three bedrooms, one bathroom, a kitchen, a living room and a dining room. Each of these was represented by a zone, which in turn consisted of sub-zones. Each zone and sub-zone had a name and each tag had a unique identification code (ID) that enabled the system to identify the occupant and his/her location (Spataru and Gillott, 2012).

Each sub-zone was associated to an object, for example the area around a TV, heater or light switch. Passive RFID tags were placed on chairs (since these could be moved around the house), onto the light switch to determine when and who was switching the light on/off (Spataru and Gillott, 2012).

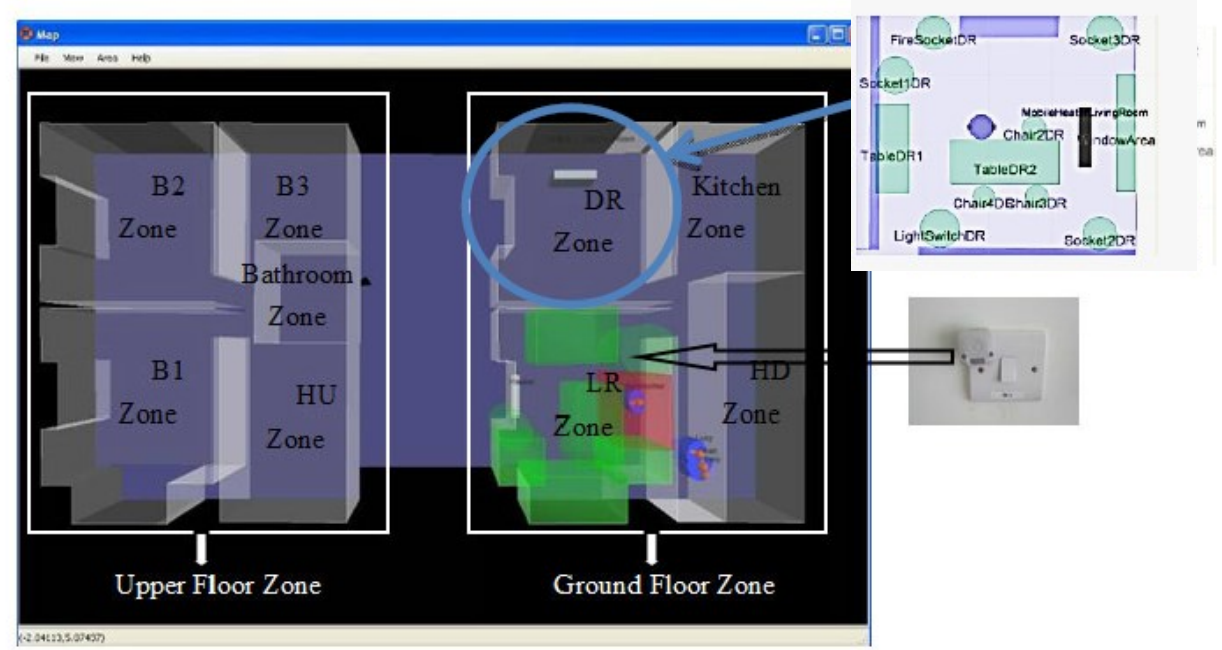

Figure 2.8 Schematic visualization of the zones considered in the test house (Spataru and Gillott, 2012)

The occupants' patterns were used in combination with the energy consumption to determine their individual energy consumption. Based on zone description and energy consumption status through the detailed monitored system for each socket, an activity was queried for each sensor event. The measured activities were: sleeping, cooking, watching TV, shower/bath, use of 
computer, laundry, vacuum cleaning, and switching lights on/off. This information was beneficial to determine where the energy was consumed in excess and how occupants behaved to maintain their comfort, as well as the result of the energy retrofit changes to the house (Spataru and Gillott, 2012).

\subsection{Energy Simulation}

\subsubsection{Simulation and Measurement of Energy Consumption}

Measurement and simulation are two approaches to obtain the energy use of buildings. While measurement can provide more accurate data, it can be time consuming and costly. On the other hand, simulation is a faster and more cost-effective way to estimate energy consumption although the results are not as accurate as the measured data. Therefore using both approaches is suggested as they supplement each other (Hong et al., 2013).

However, due to the difficulty in obtaining real-time accurate occupancy data, many of researchers focused on using pre-determined schedules. Among the studies that evaluate their energy saving potential, only around $20 \%$ evaluated their solution in a real test-bed or actual experiments, while some others dealt with energy consumption apportionment or prediction instead of controlling appliances (Nguyen and Aiello, 2013).

\subsubsection{Building Energy Simulation}

Building energy simulation means using computer-based tools to simulate energy usage of a building through a period of time, such as a specified day, an entire year, or whole building life (ASHRAE, 2002).

Building information, including building structure and orientation, characteristics of external surfaces, characteristics and operation schedules of HVAC system, characteristics and operation 
schedules of lighting system, location or weather data, and other related information, would be input in simulation programs. Many kinds of software include both annual energy consumption and different energy consumption (e.g. cooling, heating, lighting, hot water, equipment) break down for different periods of the year in their simulation results. Usually annual, monthly, daily, and hourly change curves of energy consumptions, as well as energy usage contribution are calculated. In some tools, related parameters like energy cost and $\mathrm{CO}_{2}$ emission are given in final report; while in others, simulation results are compared with code requirements (Shalini, 2011). The estimation of a building's energy consumption is carried out by building simulation tools, such as Energy plus, eQuest, Ecotect, IES $<\mathrm{VE}>$. These tools are helpful in designing energy saving at both the equipment level (e.g. lighting, heating, ventilation and air conditioning (LHVAC) systems) and building level. One crucial information for the estimation of the energy demand of a building is its occupancy. When a building is occupied, the individuals modify the indoor environment, as human beings emit heat and pollutants. They also interact with the building, such as adjusting lighting and air conditioning systems, to enhance their comfort. They also operate electronic appliances, such as computers, which consume energy and produce heat. A model that predicts the presence or absence of occupants in a building helps the simulation tool to produce more accurate energy consumption estimation ( $\mathrm{Yu}, 2010)$.

\subsubsection{Energy Simulation Software}

Procedures and methodologies to baseline energy use in commercial buildings began to appear in the 1980's and early 1990's. Since then, a number of modeling toolkits and software have been developed that are useful in developing performance metrics for buildings. Over the past fifteen years or so, a few researchers have attempted to use short-term monitoring for the purpose of base lining building energy use (Singh, 2011). 
In order to estimate the energy consumption of a building, it is important to understand how energy is distributed throughout the building and how each of the building elements contributes to the total energy consumption of the building. Building simulation can be an effective tool, both for understanding how a building consumes energy and for assessing building schedule strategies aimed at improving building energy efficiency. The procedure involves using daily or hourly whole-building energy use to develop a number of feasible predicted models for the building (Singh, 2011).

Energy simulation can couple climatic data and occupancy profile with building loads, such as HVAC, the number of occupants and their activity levels, and lighting levels. Therefore, having detailed occupant profiles will improve the accuracy of the simulation. Building energy simulation tools have been developed rapidly during the recent years. The US Department of Energy has listed 129 different kinds of software. Most common software programs that are widely used in the world are; DOE-2, EQUEST, EnergyPro, EnergyPlus, IES $<$ VE $>$, ECOTECT, Green Building Studio (GBS), HEED, Design Builder, and DeST (Attia et al., 2009).

\subsubsection{Energy Modeling}

An energy simulation tool is usually composed of three parts: geometric design module to create the geometry of the model, parameter input module, and calculation module. It is sometimes difficult to create accurate geometric models for a complicated project in simulation tools with their own geometric design modules (Attia et al., 2009).

To analysis the energy consumption and to get the better optimized energy use, it is important to understand the impacts of the energy model. These energy models are used to predict the building's energy consumption more accurately and to help reduce the energy consumption. The energy consumption of a building depends on different issues such as lighting, HVAC, and 
occupancy behavior and their interaction with the appliances, therefore in exploring the use of energy in a building, all energy related issues must be taken into account (Krygiel and Nies, 2008).

All simulation software systems need a 3D geometric model for energy calculation. There are two ways to create the $3 \mathrm{D}$ geometric model. One is to create a simplified geometric model in the energy simulation software based on original architectural drawings, including floor plans and elevations. Another is to use a professional design tool, like AutoCAD or Revit to create the model and import it into the simulation software. There is the ability to transfer the essential parts of project from one application to another application with different formats, such as Green Building Extensible Markup Language (gbXML) or Industry Foundation Classes (IFC). All geometry model and some of the locations and building type settings can be exported into a gbXML schema which determines project location, building envelope, room volumes, and any application-specific settings files. Then, the model can be imported into an energy modeling tool such as IES $<\mathrm{VE}>$, Ecotect, eQUEST or GBS. The selection of the software depends on the skill level, accuracy of the results, time available, and the current phase of the project (Krygiel and Nies, 2008).

The most important advantage of creating a geometric model in the energy software is that it will make the modeling procedure more smoothly with fewer errors. However, since many simulation tools have simplified 3D modeling module in their interface, while target buildings might have complicated shapes and structures, it may be impossible to create the geometry models within the simulation tools (Krygiel and Nies, 2008). To create an energy model, the first step is to have a well-built model. Information needed in the model, including structures, envelope characteristics, HVAC systems, lighting systems, occupancy profile, and other related 
information are usually gathered from different data sources, calculated, or estimated. With the model, the first draft of the simulation results could be calculated (Singh, 2011).

\subsubsection{Probabilistic Data Simulation}

Experts who recorded and analyzed the day-to-day energy-related actions of building occupants tended to reach the same general conclusions: that occupancy behavior has a significant impact on building energy requirements; that this behavior is highly variable and therefore hard to predict; and that more realistic occupant models are needed. Therefore, buildings must be designed to support the day-to-day activities of their occupants since early design decisions are widely believed to have a disproportionately large impact on a building's ultimate energy requirements. Various modeling approaches have been developed for use in building energy performance simulations to predict occupancy characteristics in different types of buildings.

A bottom-up modelling approach with a set of calibration methodologies was proposed by Wilke et al. (2012) to predict residential building occupants' time-dependent activities for use in dynamic building simulations. The stochastic model to predict activity chains was calibrated using French time-use survey data (of 1998/1999), based on three types of time-dependent quantities: (1) The probability to be at home; (2) The conditional probability to start an activity whilst being at home; (3) The probability distribution function for the duration of that activity. The behavior of the individual agents in the model was first calibrated using a generic approach, where every individual was assumed to behave the same. A refinement was then presented to account for variations in the behaviors of sub-populations, having specific individual characteristics.

Furthermore, a statistical approach was introduced for the modelling of transitions between two successive activity types as a Markov process. The models were then validated using a cross- 
validation technique, and their predictive performance was compared at an individual level, as well as for aggregated (sub-) populations (Wilke et al., 2012).

Bourgeois (2005) showed how simulations, which distinguish between individual occupants, can be used to predict the performance of both manual and automated lighting control systems. Page (2007) proposed a method for simulating rooms as they alternate between unoccupied and occupied states. Page's method represented an example of machine learning and a clear departure from the static 24 -hour profiles. These static 24-hour profiles were created based on the randomly generated state transitions probabilities derived from measured occupancy data. The profiles were used to estimate occupancy in most energy modeling tools.

Tabak (2008) developed a model of activity and location schedules using a system of User Simulation of Space Utilization (USSU), to generate movement patterns that provide a representation of human activities in office building spaces. Tabak demonstrated an alternative to machine learning: a complex scheduling algorithm that accounted for the role of each occupant in an organization, the tasks they performed alone and with other occupants, and the layout of a building (Tabak, 2008).

Hoes et al. (2009) combined the work of Tabak (2008) and Bourgeois (2005) and showed that increasing the level of details of an occupant model may significantly change energy use predictions and real energy consumption.

Goldstein et al. (2011) tried to address the needs of designers. They proposed a machine learning method which randomly generated schedules of occupant activities as output reflecting both realworld measurements and predicted design-level inputs.

$\mathrm{Yu}$ (2010) applied a genetic algorithm to learn the behavior of an occupant in single person office based on motion sensor data. The learned rules predicted the presence and absence of the 
occupant with $80 \%-83 \%$ accuracy on testing data from five different offices. The rules indicated that the following variables may influence occupancy behavior: (1) The day of week; (2) The time of day; (3) The length of time the occupant spent in the previous state; (4) The length of time the occupant spent in the state prior to the previous state; and (5) The length of time the occupant has been in the office since the first arrival of the day. They evaluated the rules with various statistics.

\subsection{Summary and conclusions}

This chapter reviewed the concepts, techniques, standards, and main technologies that are used in the current research. The literature review included the information about RTLSs technologies, RFID based localization techniques and smart metering. RTLSs have been applied in previous studies for identifying, monitoring and localizing purposes; whereas smart metering is a common methodology used to measure the energy consumption of equipment.

However, occupancy activities and behaviors have a large impact on energy consumed in different buildings' sectors (i.e. residential, offices and retail sectors). Therefore, to evaluate the occupancy behavior on energy consumption, there are limitation in these studies regarding the accuracy and the lack of statistical data. Significant amount of energy spent in these buildings could be saved by regulating installations and IT equipment according to actual needs. In order to realize this approach, occupant activities and behaviors are required as the most important input for occupant simulation profile. 


\section{CHAPTER 3 Requirements Analysis and Proposed Methodology}

\subsection{Introduction}

As explained in Section 2.3 occupancy presence and behavior in buildings have been shown to have large impacts on space heating, cooling and ventilation demand, energy consumption of lighting and IT equipment. Past research has shown that the use of real-time occupancy information for control of various devices like artificial light, HVAC devices, and IT equipment can save significant energy.

Evaluating the impact of occupancy behavior on energy consumption, as discussed in Chapter 2, requires deploying technologies, monitoring, data collection and analysis of collected data via appropriate applications. A framework is proposed and applied in the test bed to overcome the technical requirements of the solution. In this regard, an appropriate spatiotemporal data fusion model is required to accurately correlate the results of the wireless networks to infer the most suitable response. Figure 3.1 demonstrates the steps followed in the proposed method.

This chapter includes an introduction to the problem statement which is explained in Section 3.1. Tracking, identification, and monitoring technologies and requirements of the technologies deployment are introduced and described in Section 3.2. A test design applied in an office building is proposed in Section 3.3 based on the discussed criteria and requirements in Section 3.2. Test results are analyzed and visualized in Section 3.4. Energy consumption simulation and the energy saving based on proposed method are explained in Section 3.5. This chapter concludes with summary and conclusions in Section 3.6. 


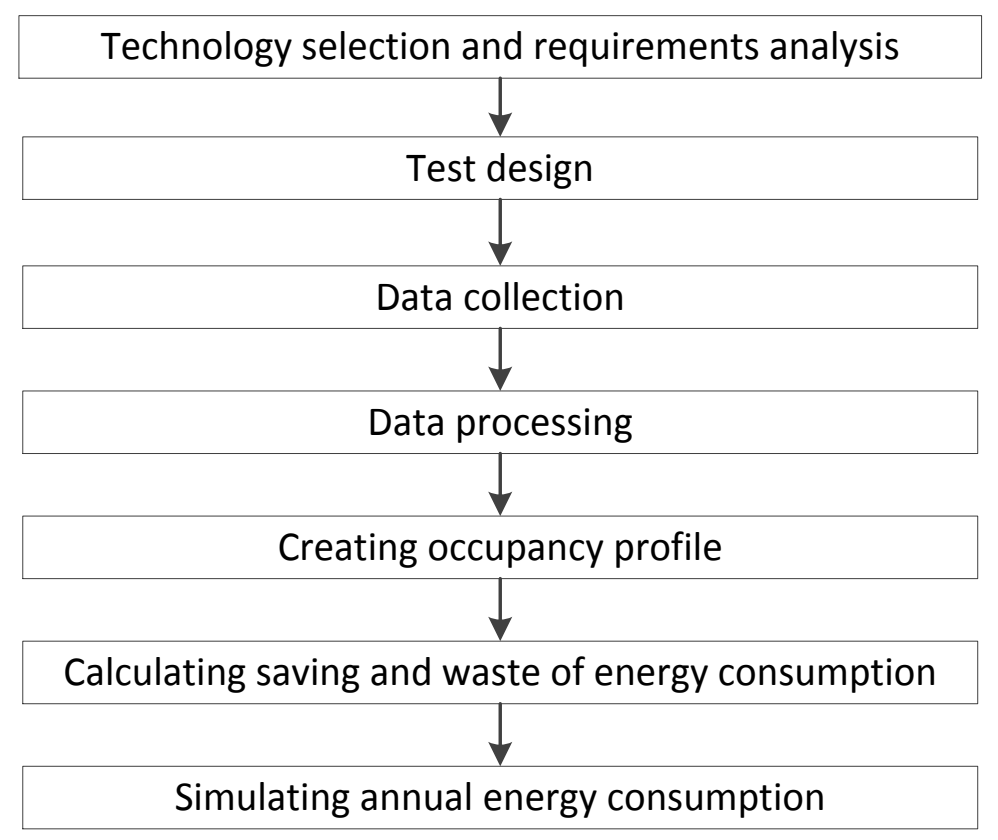

Figure 3.1 Proposed method steps

\subsection{Technology Selection and Requirements Analysis}

An experimental study with the objective of monitoring IT equipment energy consumption and occupancy behavior is proposed in order to minimize energy consumption in office buildings. Two wireless sensor technologies are used for monitoring occupancy behavior and energy consumption of IT equipment (i.e., monitors, computers, and printers). UWB RTLS is used for occupancy location monitoring and ZigBee wireless energy meters are used for monitoring the energy consumption of IT equipment. UWB provides the identity and location of occupants at short time intervals. On the other hand, energy meters provide instantaneous and cumulative equipment energy consumption data simultaneously providing the information of how much energy is consumed by each device.

The initial cell update rate for UWB and ZigBee energy meters should be in the same range (e.g. one reading per second for each). In this test the update rate is assumed to be set in accordance with the occupants' movements' duration and energy consumption reading needs. For example, 
printing a few papers may take few seconds and occupants' movements within the room to pick up printed papers may also take few seconds. In addition, there is always the possibility of having missing data during the test so in order to increase the accuracy, the average of two sequential readings are considered in the test.

\subsubsection{UWB Technology for Occupancy Monitoring}

UWB is a wireless solution capable of delivering a system that provides accurate location data with high reliability. The key physical components of the UWB are tags, sensors, network components, and platform server, which are described as follows (Ubisense, 2013).

\subsubsection{UWB Technology}

The UWB sensors, slim tags, and compact tags are shown in Figures 3.2(a), (b), and (c), respectively. The sensors detect the signals and calculate the positions of the tags. The sensor units are placed above the detection area in which the tags are tracked. They must be provided with power, networking and timing cable connections. Sensors are arranged to cooperate in one or more location engine cells. Each cell has a single master sensor and a number of slave sensors. The master and slave sensors are physically the same. The sensors are measurement devices containing an array of antennas and UWB radio receivers to detect UWB pulses from Ubisense tags, allowing the Location System to position tags in 3D with the accuracy of up to $15 \mathrm{~cm}$ (depending on system configuration and environment). In this research, one sensor is configured to be master and the other three are slaves. The installed sensors are connected to the Ethernet switch with a network cable. A timing cable should connect each slave sensor to one port on the master sensor. Sensors can be powered using Power over Ethernet (PoE) (Ubisense, 2013). 
Ubisense tags can be found in a number of types such as slim tags and compact tags which are illustrated in Figures 3.2(b) and (c).

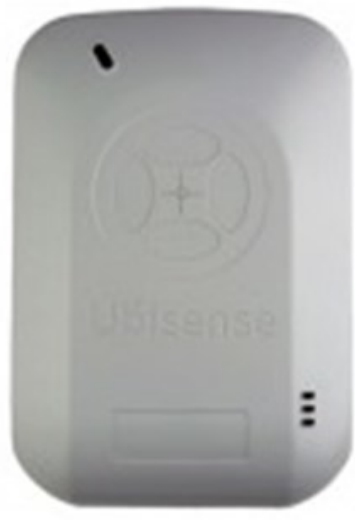

(a)

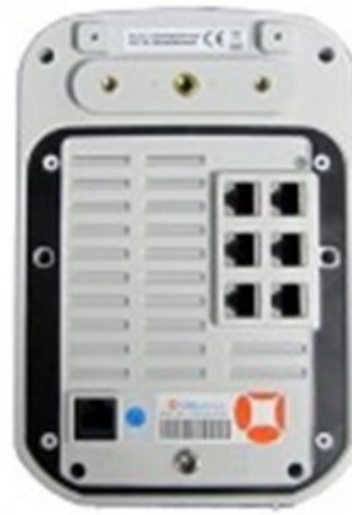

Figure 3.2 (a) UWB sensor, (b) UWB slim tag, (c) UWB compact tag (Ubisense, 2013)

They have a unique ID transmitting location radio signals to sensors. The tags must be attached to the occupants in a way that does not disturb their comfort. For that reason, slim tags were selected to be worn by office occupants as a necklace. UWB tags are assigned to subjects in the Ubisense Site Manager application to report their real-time position. The location engine cell divides time into time slots and allocates appropriate time slots to the tags according to their requested update rate.

\subsubsection{UWB Requirements}

UWB RTLS has the following requirements:

1) Accuracy: Accuracy is an essential requirement for the location-aware services to make them reliable. The UWB location system is capable of measuring both Angle-of-Arrival (AoA) and Time-Difference-of-Arrival (TDoA) of the tag's signals, enabling it to generate accurate 3D tracking information even when only two sensors can detect the tag. 
Data filtering should be applied to reduce errors in near real time and to remove noisy location data to improve the accuracy (Rodriguez, 2010).

2) Real-time response: If the number of tags in an RTLS cell exceeds a specific limit for a pre-determined update rate, locating latency would appear. With a large number of tags in a sensor cell, the update rate of tags will decrease to allow the system to cover all tags with the fixed total number of time slots. The more tags in the system, the bigger the slot interval should be selected, and the lower the update rate. A specific update rate can be set for an individual tag or a group of tags. One consideration when setting the update rate is the moving velocity of the occupant (Rodriguez, 2010).

3) Range: For the office building application, the UWB RTLS is used between fixed sensors and potentially mobile tags and is functional in metallic and furnished environments with populous interference, which cause the signal multipath problem. UWB, being immune to the multipath effect, offers the highest accuracy for the maximum indoor range (Rodriguez, 2010).

4) Visibility: To increase the visibility between the tags and sensors and to decrease the errors, placement of the sensors should avoid the blind spots and achieve the optimum coverage while the number of the sensors can improve the visibility (Rodriguez, 2010).

5) Power requirements: Although in the office building there is no concern for the stable power source, generally speaking the sensors should be connected to a stable power source for precision measurements. The tags require a battery which life depends on the update rate established for the system. The tag's update rate can be dynamically and automatically varied depending on the activity of the tag. If the tag is moving quickly, a high update rate can be assigned for best tracking; if it is moving slowly the update can 
be reduced for best battery lifetime. When stationary, tags go to sleep mode to conserve power, and an in-built motion detector ensures that the tag transmits again as soon as it is moved (Rodriguez, 2010).

6) Networking requirements: The sensors can be connected by cables (to improve the accuracy) or wirelessly to the location server. Both data cables and timing cables are needed for a wired system. The wireless system depends only on the AOA calculations as wireless communication is not fast enough to support TDOA. The decision about the type of the network (wired vs. wireless) has a direct impact on accuracy. In this test, sensors were fixed and connected to each other by timing cables (Rodriguez, 2010).

\subsubsection{ZigBee Technology and Requirements for Energy Monitoring}

ZigBee is a global wireless technology which connects different devices to work together and enhance everyday life. ZigBee home automation offers a global standard for interoperable products enabling smart homes to control appliances, lighting, environment, energy management and security, as well as the expandability to connect with other ZigBee networks (ZigBee Alliance, 2013).

Figure 3.3 demonstrates how these products make it easy for utilities and governments to deploy Smart Grid solutions that are secure, easy to install and consumer friendly. The Smart Grid provides consumers with the information they need to monitor and lower their utility bills and to manage their energy consumption in real time.

A Home Area Network (HAN) of Smart appliances that support the ZigBee Smart Energy standard connect the consumer with the Smart Grid. This standard ensures integration and interoperability with smart energy devices. Appliances can be "smartened" and joined to the HAN by connecting or plugging them into a smart plug (ZigBee Alliance, 2013). 


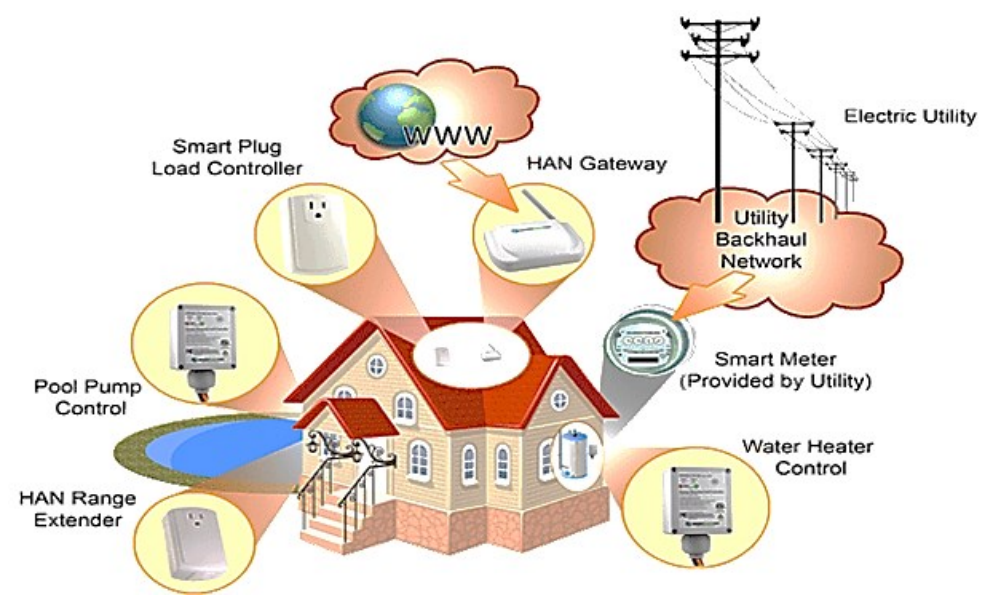

Figure 3.3 ZigBee network and products (ZigBee Alliance, 2013)

Examples of the ZigBee gateway and an energy meter are shown in Figures 3.4(a) and (b), respectively. ZigBee gateway provides a means to access the ZigBee network remotely via the Internet; whereas the ZigBee energy meters enable remote control and energy monitoring of appliances. Energy meters join the network as a ZigBee router and then facilitate the joining of other devices thus extending the entire network (Smartenit, 2013).

ZigBee networks are composed of several device types: ZigBee coordinator, ZigBee routers and ZigBee end devices. Coordinators control the formation and security of networks.

Routers extend the range of networks. End devices perform specific sensing or control functions. Manufacturers often create devices that perform multiple functions, for example a device controls a light fixture and also routes messages to the rest of the network (ZigBee Alliance, 2013).

ZigBee technology has the following requirements:

1) Gateway: Every ZigBee automation network needs one "brain" or a "coordinator" to set up the network. A gateway is the "brain" of an automation network. The brain provides 
the means for ZigBee smart devices to communicate with each other and provides remote access to directly monitor and control smart devices as well as schedule automated control based on time or device state (Smartenit, 2013).

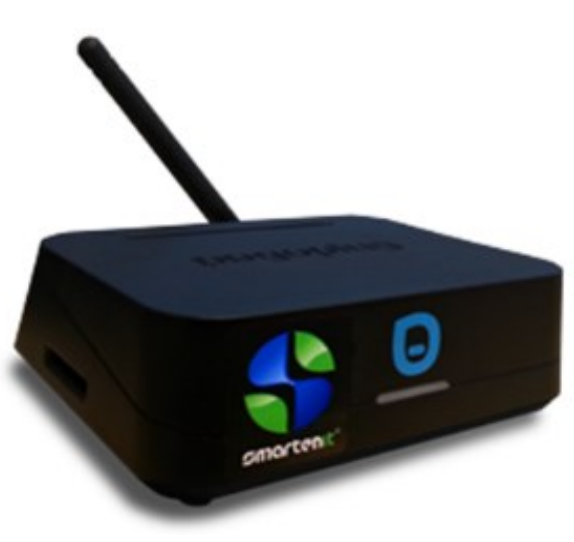

(a)

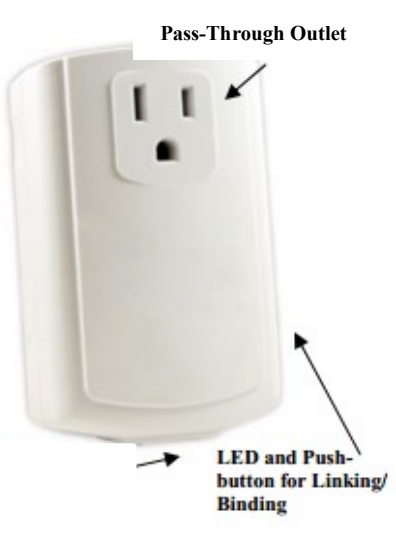

(b)

Figure 3.4 (a) ZigBee gateway, (b) ZigBee energy meter (Smartenit, 2013)

2) Line of sight: The ZigBee technology is capable of setting up a network with a limitless number of devices which have to meet the line of sight requirement with the gateway. Range extenders can be used to increase the range in the network (Smartenit, 2013).

3) Information storage: Additionally, the gateway is able to store information related to the network and offer a display of this information on an interface including energy consumption information (instantaneous and cumulative), smart plugs Mac ID, and network topology which is beneficial to maintain timers and events independently of a PC (Smartenit, 2013).

4) Networking: The gateway provides the bridge between an Ethernet connection and a wireless or hybrid home/building automation network. It is accessible from anywhere including the Internet through built-in web services and there is no need for a dedicated PC. The fully integrated gateway bridges modern home automation and computer networks, Local Area Networks (LAN)s or WANs, and operates independently of a PC 
which can be used to monitor and control appliances from any location using PCs, touch screens, and so on.

Figure 3.5 illustrates three types of topologies that ZigBee supports: star topology, peer-topeer topology, and cluster tree.

1) Star Topology: In the star topology, the communication is established between devices and a single central controller, called coordinator. The coordinator may be mainspowered while the devices will most likely be battery-powered. Applications that benefit from this topology include home automation, PC peripherals, toys and games (Ergen, 2004).

2) Mesh Topology: In mesh topology, there is also one coordinator. In contrast to the star topology, any device can communicate with any other device as long as they are in range of one another. The ZigBee wireless energy meter which is selected for this test is a mesh network. The mesh network can be ad hoc, self-organizing and self-healing. Applications such as industrial control and monitoring, wireless sensor networks, asset and inventory tracking would benefit from such a topology. It also allows multiple hops to route messages from any device to any other device in the network. It can provide reliability by multipath routing (Ergen, 2004).

3) Cluster-tree Topology: Cluster-tree network is a special case of a peer-to-peer network (Ergen, 2004). 


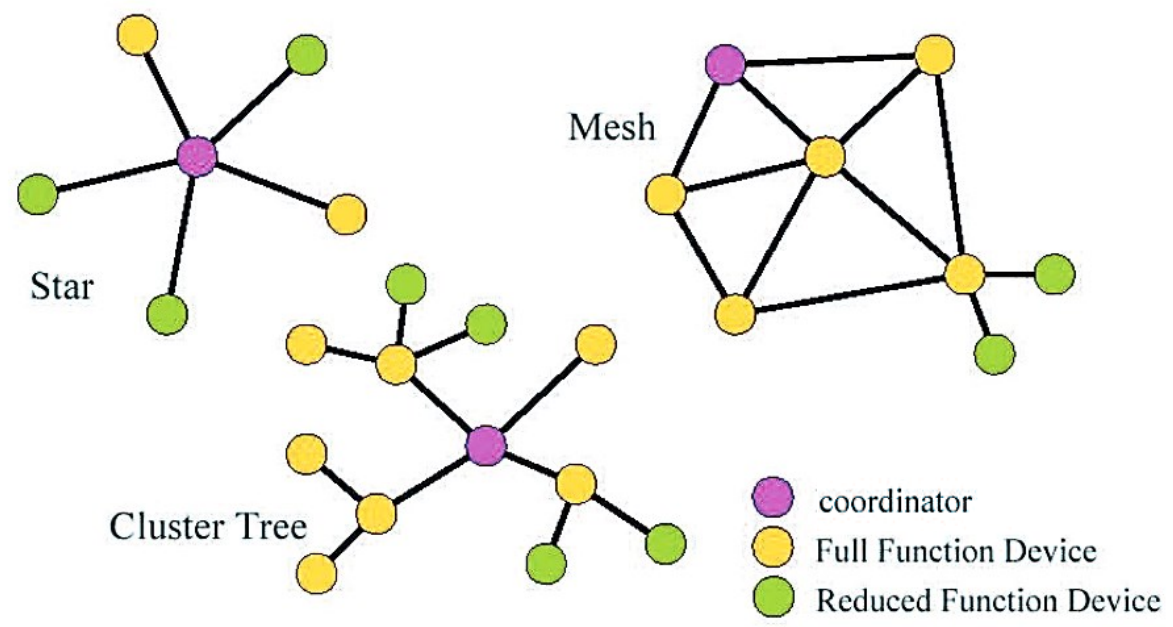

Figure 3.5 ZigBee network topology models (Ergen, 2004)

\subsection{Test Design}

Two types of wireless sensors are used for monitoring occupancy location and energy consumption of small office IT equipment (i.e., monitors, computers, and printers). RTLS is used for occupancy location monitoring and ZigBee wireless energy meters are used for monitoring the energy consumption of office equipment. UWB provides the identity and location of occupants at different points of time and participants are required to wear UWB tags during the test. On the other hand, energy meters provide instantaneous and cumulative equipment energy consumption data simultaneously providing the information of how much energy is consumed by each device. Energy meters should be attached to the IT equipment in the office (i.e. monitors, computers, and printers) which are in use by the occupants.

Figure 3.6 illustrates the different data sources that can be used to analyze the occupancy behavior based on the locations of the occupants of an office and their IT equipment and energy consumption. 


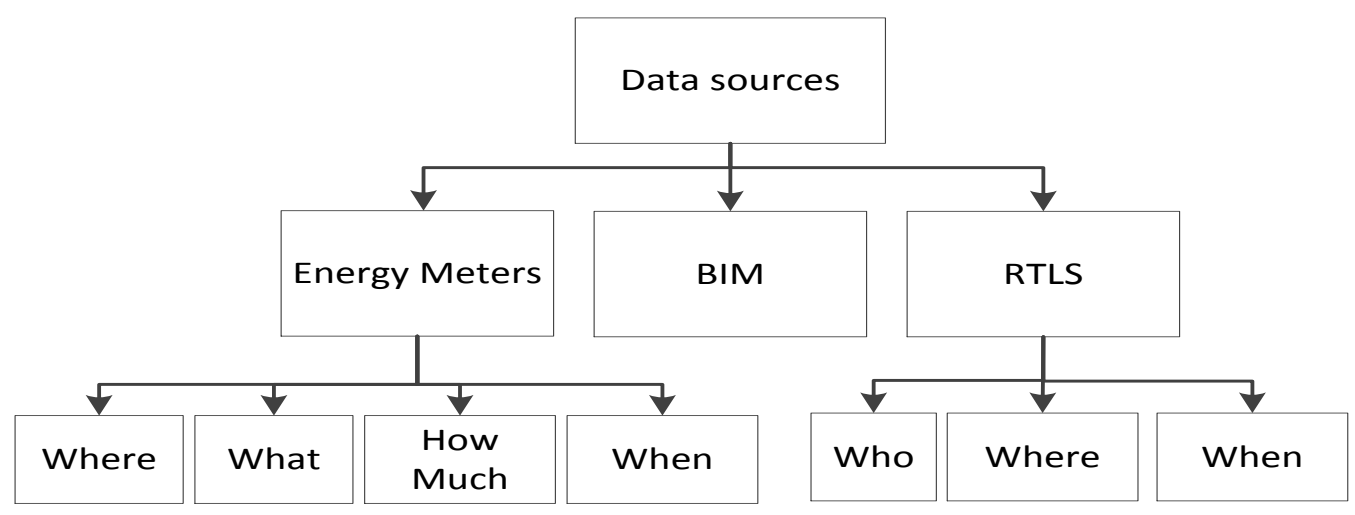

Figure 3.6 Data sources (Masoudifar et al., 2014)

The following are the main data sources: (1) An UWB provides the identity and the location of the occupants at different points of time. By analyzing the UWB RTLS data, the following question can be answered: How many people are in the office for a specific time? Who is where and for how long at any time? In other words we can obtain occupants' detection, identification and localization. (2) Energy meters provide real-time equipment energy consumption data simultaneously providing the answers for the following question: Which device is using how much energy at specific times? By combining the location data and energy consumption data, the answers for the following questions can be also found: Who is doing what? How much energy is used by each occupant? How much energy is wasted by each occupant? (3) The BIM model of the office includes architectural elements, furniture, IT equipment, and wireless sensor networks to evaluate occupancy behavior. Each occupant is assigned to a specific zone within the office that includes the IT equipment used by that occupant. Occupancy profiles can be constructed from the data gathered from the UWB sensors. 


\subsection{Data Processing}

\subsubsection{Data Pre-processing}

The data can be fused to link the occupants with their energy consumption. Before data fusion, UWB and ZigBee energy meters data must be separately processed to provide the location and energy measurement data, respectively. Furthermore, some other pre-processing is required to provide the UWB and ZigBee energy meters datasets which are aligned in terms of time, frequency, KW/KWh readings, and coordinates. This can be done by data synchronization, averaging, filtering, and filling missing data. At each data collection step, several values from various tags are sensed and logged by the readers. Some received values can be outside the expected range and show systematic errors. These readings should be filtered out from the logged data. Moreover, there may be missing readings during data collection which can be filled by data interpolation. Then the average value for each data collection step should be calculated to be used for matching the UWB and ZigBee readings. The pre-processing assures providing reliable data for the analysis.

UWB and ZigBee energy meters log files are analyzed in Microsoft Excel software for identifying the tags and plugs data accuracy and their update rates. According to the Excel visualization results explained in Section 4.5, UWB tags demonstrated some location errors; and therefore their data are pre-processed. On the other hand, ZigBee energy meters results in Excel demonstrated that there are some missing data and false readings; therefore the data are preprocessed. Then the UWB and ZigBee energy meters results are ready to be synchronized. 


\subsubsection{Visualization}

The real-time occupancy location and IT equipment pre-processed data can be visualized. The visualization provides a method for quickly analyzing the real-time collected data. One of the key factors in dealing with real-time data collection for visual analytics in this research is the spatiotemporal dimension of time-varying data and to present this data for different time intervals. The proposed visualization method should go back and forth in time and view the visualization related to different time intervals during the test period.

To present the spatial dimension of the time-varying data, daily occupancy maps are created to demonstrate occupants' locations in the office based on UWB coordinates (X, Y) during each day. As shown in Figure 3.7(a), each symbol indicates one of the occupants and each point indicates the location of a specific occupant at a certain time. However, it is not possible to determine the time intervals at which each occupant is in the office from these occupancy maps. Therefore, occupancy charts are developed to illustrate occupants' presence at different time intervals of the day which is shown in Figure 3.7(b).

Moreover, similar charts can be used to represent daily and weekly occupancy (temporal visualization of the collected data). Occupancy daily and weekly charts demonstrate occupants presence at different time intervals of the days which can be used to create occupancy profiles. 


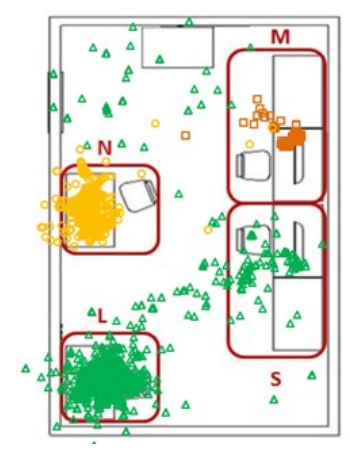

(a)

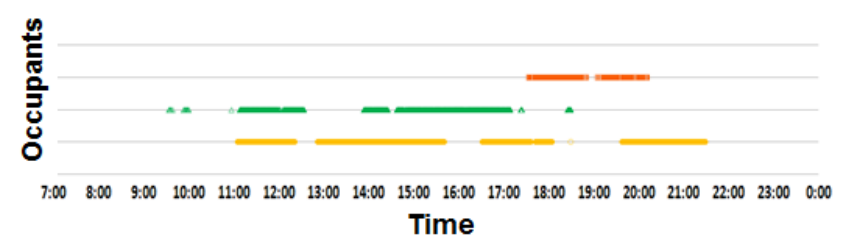

(b)

Figure 3.7 Spatial temporal data visualization: (a) occupancy map, and (b) occupancy chart

\subsubsection{Statistical Occupancy Profiles}

As explained in Section 2.3, the occupancy profile is one of the driving factors behind discrepancies between measured and simulated energy consumption of buildings. Therefore, optimizing the occupancy profile can result in minimizing building energy consumption. Occupancy profile demonstrates occupants' hourly presence and absence in the office during week days and weekend days. Occupants' location data gained from UWB are used to create occupancy profile. Assuming that the occupants' presence and absence pattern is almost constant during the year, the results from short-time monitoring can be used to simulate the long-term energy consumption. Profiles are created on an hourly basis since occupants may leave the office for a short period of time (to talk on their cellphone, to go to the washroom, and so on) and within these short periods of time they may not turn off their equipment since they are in the middle of their work. Two different types of the occupancy profiles are created: (1) the overall occupancy profile, which demonstrates total number of the present occupants in the office for each day of the week, and (2) the probabilistic individual occupancy profile, which explains the possibility of each of the occupants presence possibility during weekdays and weekend days which is useful to identify the approximate energy consumption of each occupant. To create the the overall occupany profile, location data from UWB are categorized based on the UWB tags 
IDs (each of the UWB tags are assigned to one of the occupants). Then the number of the occupancts are counted on an hourly basis. To simplify the calculation, if there was any reading for the occupant in an hour, that hour would be counted for him/her. At the end, an overall occupancy profile is created for each day showing the possibility of the number of the occupants in the office on an hourly basis. The formula which is used to calculate the overall occupancy profile is explained below.

$P_{d}^{t, t+1}=\frac{1}{n} \sum_{o=1}^{n} P r_{o, d}^{t, t+1}$

Where $P_{d}^{t, t+1}$ : the probability of the presence of all occupants between time $t$ and $t+1$ on day $d$, $\operatorname{Pr}_{o, d}^{t, t+1}:$ the presence of the occupant $o$ between time $t$ and $t+1$ on day $d$ which is 0 or $1, o$ : occupant number, $t$ : hour of the day, $n$ : total number of occupants, and $d$ : day of the week.

Similar to the overall occupancy profile, location data from UWB are categorized based on the UWB tags IDs to create probabilistic individual occupancy profile. Then, the total time that each occupant spent in the office is calculated on an hourly basis. To simplify the calculation, if there was any reading for the occupant in an hour, that hour would be counted for him/her. At the end, a probabilistic profile is created for each occupant that demonstrates the possibility of the presence for each occupant in the office on hourly basis. This procedure is summarized in Figure 3.8 .

The formula which is used to calculate the probabilistic individual occupancy profile is explained below. This procedure is applicable to calculate both weekdays and weekend days probabilistic individual occupancy profile.

$P_{o}^{t, t+1}=\frac{1}{m} \sum_{d=1}^{m} P r_{o, d}^{t, t+1}$ 
Where $P_{o}^{t, t+1}$ : the probability of occupant $o$ presence between time $t$ and $t+1$ on an average day, $\operatorname{Pr}_{o, d}^{t, t+1}:$ the presence of occupant $o$ between time $t$ and $t+1$ on day $d$, which

is 0 or $1, o$ : occupant number, $t$ : hour of the day, $m$ : total number of the days, and $d$ : day of the week.

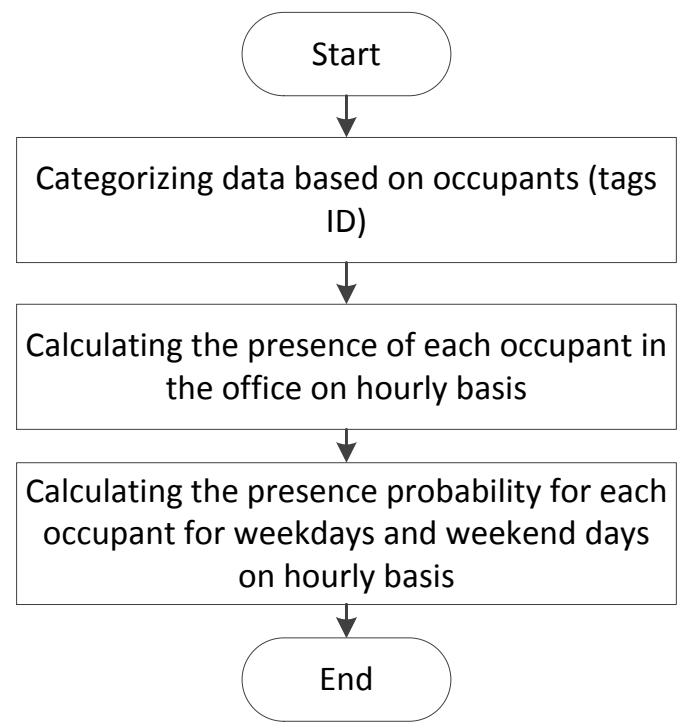

Figure 3.8 Procedure to calculate the probabilistic individual occupancy profiles (Masoudifar et al., 2014)

\subsubsection{Data Fusion for Calculating Energy Waste}

As shown in Figure 4.1(b), the office area is divided into different zones. Each of the occupants is assigned to a specific zone within the office. Zones are defined based on the occupants' work spaces including the IT equipment which is used by that occupant. Occupants' presence is analyzed based on the three following states: (1) the occupant is in the office and within his/her zone, (2) the occupant is in the office but not in his/her zone, and (3) the occupant is not in the office. Each of these states indicates different probability for the office IT equipment energy consumption. For example during state one, occupants almost certainly consume energy with the assumption that if the occupants are in the office and in their zones, then they are most probably 
using their IT equipment. While during states two and three, the occupants are most probably not using their IT equipment.

Figure 3.9 shows the flowchart for calculating total energy waste. In the first step, there are two possibilities, whether the occupant is in the office or not. The presence of the occupant in the office would result into one of the following scenarios: being in his/her zone or out of zone.

Next, there may be waste of energy during the times in which the occupant is in the office but not in his/her zone while the equipment is on. On the other hand, if the occupant is absent then the possibility of waste of energy may occur if the equipment assigned to the occupant is on. The above procedure should be repeated for each occupant at each time step (i.e. every minute). At the end of the analysis period, the summation of durations $T_{5}$ and $T_{7}$ is used to calculate the total energy waste related to the equipment used by each occupant.

\subsection{Energy Consumption Simulation}

The energy simulation tool is composed of different modules such as geometric design module to create the geometry of the model, parameter input module (HVAC, construction, location and weather, and so on) including office environment specification and collected data from IT equipment monitoring, and calculation module to run the simulation.

The geometry of the 3D model can be created whether in the geometric design module of the simulation software or in one of the modeling software. The geometry created in the modeling software then can be imported into the simulation software using different formats such as gbXML including project location, building envelope, room volume, and application-specific settings file. 


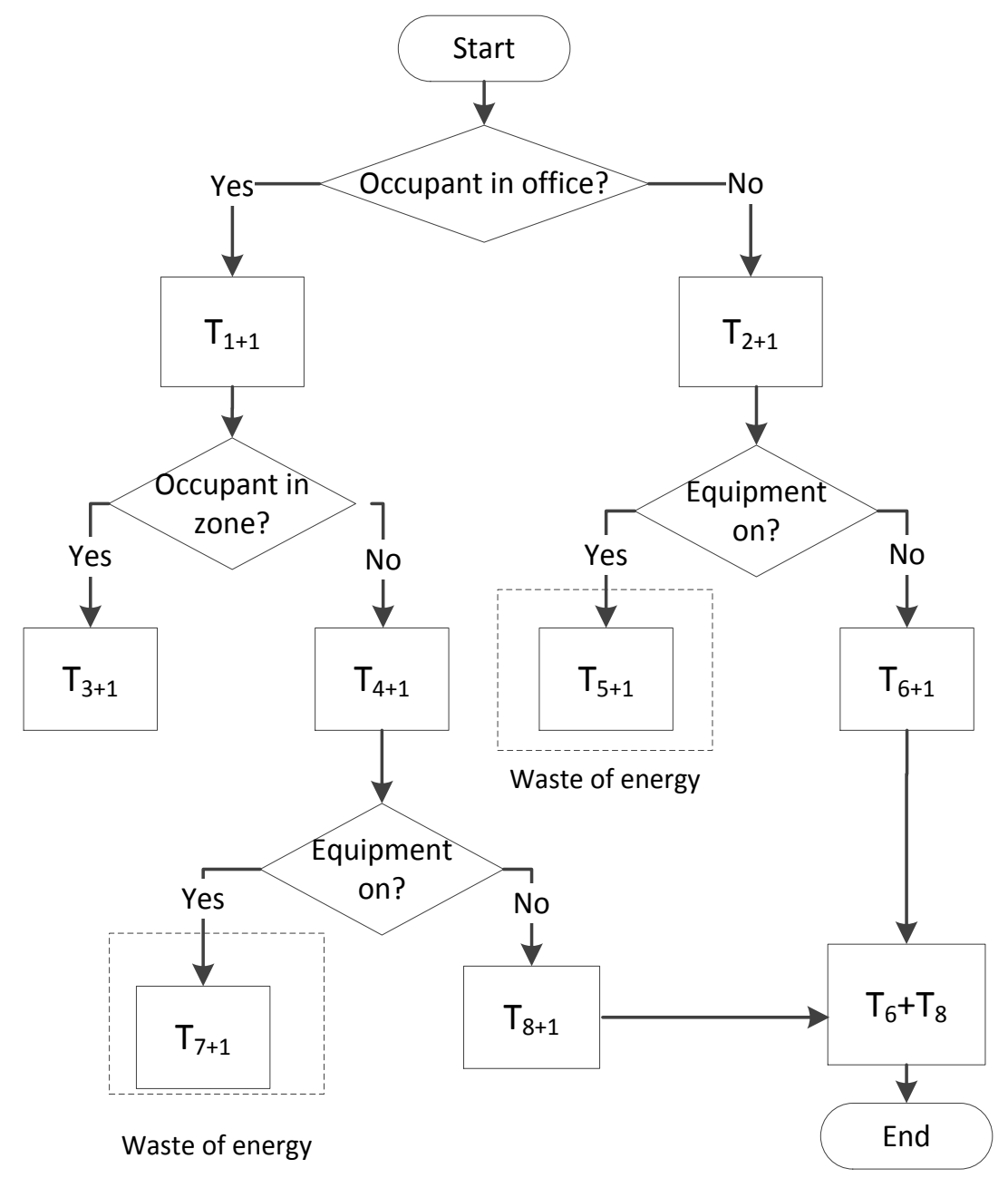

Figure 3.9 Flowchart for calculating energy waste (Masoudifar et al., 2014)

To create the energy simulation model, the building opaque characteristics, HVAC systems, lighting systems, occupancy profile, location and weather data, and other related information are specified as inputs into the simulation software. The simulation software can help calculating cooling, heating, lighting, and IT equipment energy consumption breakdowns for different period times of the year as well as the annual, monthly, daily, and hourly energy consumptions curves.

Moreover, as explained in Section 2.7, the occupancy profile can optimise the energy consumption simulation results. To assign the occupants information to the model, two different 
types of profiles are created. A simulation profile based on overall occupancy profile is created in the software to calculate the heating, cooling, and lighting energy consumption. Another simulation profile is created based on probabilistic individual occupancy profile to calculate the IT equipment energy consumption.

With the simulation model, the annual energy consumption can be calculated. The results explain the energy consumption breakdowns for heating load, lighting energy consumption, and IT equipment based on occupancy profile. In conclusion, simulation software calculates the difference between the estimated energy consumption based on occupancy profile and fixed profile energy consumption.

\subsubsection{Heating and Cooling Profile}

As mentioned in Section 3.3, the test is carried out in a small multi-occupants office where there is only one air terminal device; therefore it is assumed that if only one of the occupants enters the office, the heating system should start working. It means that the heating is whether ON or OFF. Occupancy profiles (explained in Section 3.4.3) are created based on occupants' presence gathered by UWB. The occupancy profiles show the number of the occupants and their identification in the office. Two different occupancy profiles are created named overall occupancy profile and individual probabilistic occupancy profile. The overall occupancy profile provides the information about the presence of any occupant in the office at a specific time or not. Based on the overall occupancy profile, daily profiles are defined as series of values on an hourly basis specific for different times of weekdays and weekend days. 
As shown in Figure 3.10(a), the number of the occupants in the office at 8:00 AM on Monday was zero while on the same day at 4:00 PM there were two occupants in the office assuming the total number of occupants of an office is four.

The values of zero and one are assigned to each hour of the day, depending on whether there are occupants in the office or not, respectively. If there was at least one occupant in the office, it means that $100 \%$ of the maximum value of the heating was demanded; and if there was none of the occupants in the office, it means that $0 \%$ of the maximum value of the heating was demanded and in use. In conclusion, a profile to regulate the heating energy consumption based on the results in occupancy overall profile was defined in the software to simulate the heating energy consumption.

This profile is assigned to the office model in the software and annual heating energy consumption is calculated based on the proposed profile. Comparison between the heating energy consumption, based on this proposed profile, and a fixed profile can show the difference in the amount of the energy consumption as explained in Chapter 4.

\subsubsection{Lighting Profile}

To simulate the lighting energy consumption, it is assumed that individual lighting fixture is assigned to each of the occupants' zones. It means that if any of the occupants is in his/her zone, that zone's lighting is $\mathrm{ON}$ and in order to save energy if the occupant is not in his/her zone (or any of the occupants are not in that specific zone) the light would be OFF. 


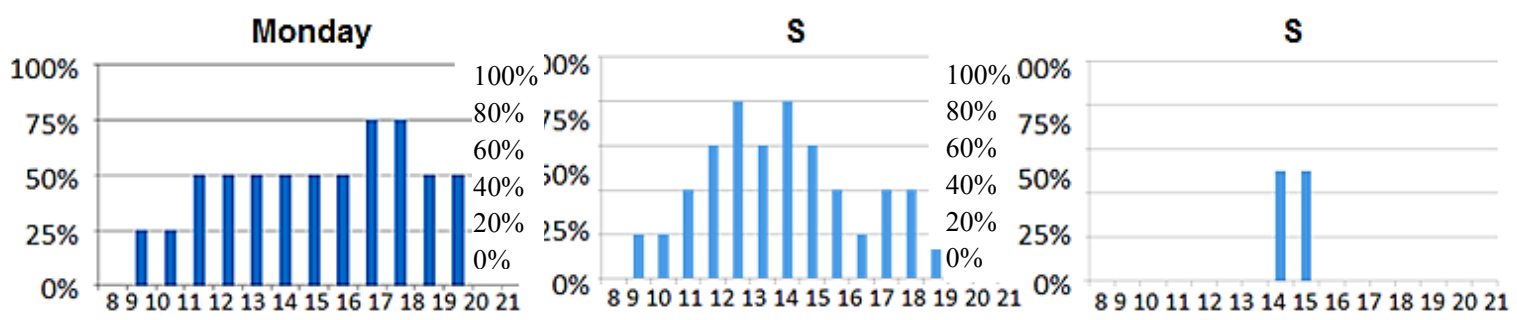

(a)

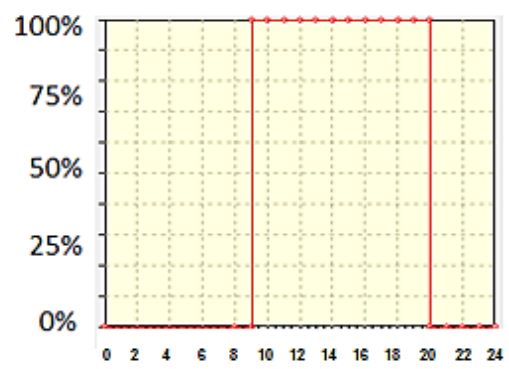

(d)

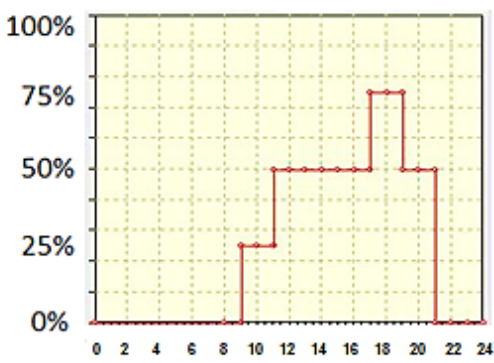

(g) (b)

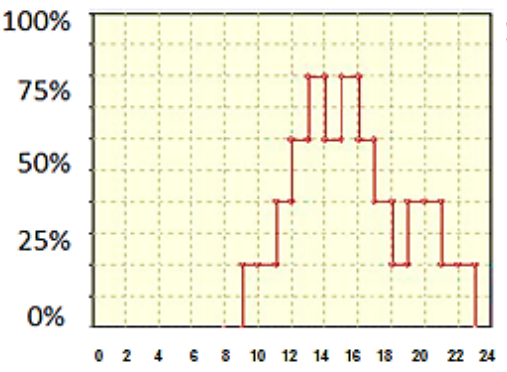

(e) (c)

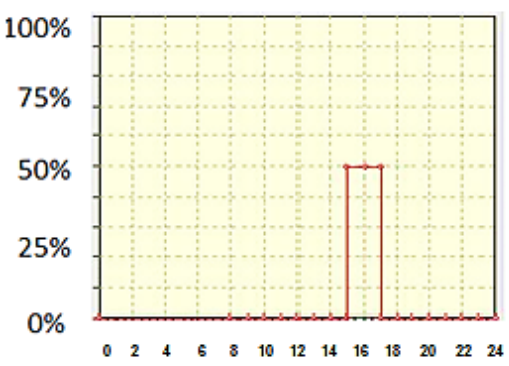

(f)

Figure 3.10 (a) Overall occupancy profile, (b) Occupant $S$ weekdays probabilistic individual occupancy profile, (c) Occupant $S$ weekend days probabilistic individual occupancy profile, (d) Heating and cooling simulation profile, (e) Occupant $S$ weekdays IT equipment simulation profile, (f) Occupant $\mathrm{S}$ weekend days IT equipment simulation profile, (g) Lighting simulation profile

As shown in Figure 10(g), the same as heating and cooling profile, daily lighting profiles are defined as series of values applying on an hourly basis at different times of weekdays and weekend days based on the overall occupancy profile. For instance, Figure 3.10(a) illustrates that the number of the occupants in the office between the hours 9:00-10:00 AM was one on Monday, it means that $25 \%$ of the maximum value of the lighting was demanded and in use; therefore the value of $25 \%$ is assigned to that period of the day. On the other hand, there were three occupants in the office on the same day between 4:00 PM and 5:00 PM. Hence 75\% of the maximum value of the lighting was demanded and in use, and consequently the value of $75 \%$ is 
assigned to that period of the day. In conclusion, a profile to regulate the lighting energy consumption based on the results in occupancy overall profile was defined in the software to simulate the lighting energy consumption. This profile is assigned to the office model in the software and annual lighting energy consumption is calculated based on the proposed monitored created profile. Comparison between the lighting energy consumption, based on the proposed profile, and a fixed profile can show the difference in the amount of the energy consumption as explained in Chapter 4.

\subsubsection{IT Equipment Profile}

Probabilistic individual occupancy profiles based on monitored data were used to create IT equipment profiles to simulate equipment energy consumption. As described in Section 3.4.3, probabilistic individual occupancy profiles demonstrate the probability of the presence in the office of each of the occupants on an hourly basis during weekdays and weekend days.

In the simulation, it is assumed that if the occupants are in the office, his/her IT equipment is in use. Based on the monitored data, the occupants spend most of their times in their own zone while they are in the office; hence to simplify the simulation, it is assumed in the profile when an occupant is present, he is in his zone and using his IT equipment. To sum up, if the occupant is in his/her zone, the IT equipment in the zone is ON; and in order to save energy, if the occupant is not in his/her zone (or any of the occupants are not in that specific zone) the IT equipment would be OFF.

As shown in Figures 10(e) and 10(f), the daily occupants' IT equipment profiles are defined as series of values (on an hourly basis) applying at different times of weekdays and weekend days on an hourly basis based on probabilistic individual occupancy profiles. Figures 3.10(b) and (c) 
demonstrate that there is the possibility of $20 \%$ that the occupant $\mathrm{S}$ be in the office between 9:00 AM and 10:00 AM during weekdays. Therefore there is the possibility of $20 \%$ of using IT equipment (it is assumed that IT equipment consumes a fixed amount of energy when it is running). Thus the value of $20 \%$ is assigned to the duration between 9:00 AM and 10:00 AM of occupant S equipment profile during weekdays. Moreover there is the possibility of about $5 \%$ that the occupant $\mathrm{S}$ be in the office between 4:00 PM and 5:00 PM during weekend days. Consequently the value of 5\% is assigned to the duration between 4:00 PM and 5:00 PM of occupant S's IT equipment profile during weekend days. In conclusion, a profile to estimate the IT equipment energy consumption based on the results in the probabilistic individual occupancy profile was defined in the software to simulate the IT equipment energy consumption. This profile is assigned to the office model in the software and annual IT equipment energy consumption has been calculated based on the proposed monitored created profile. Comparison between the IT equipment energy consumption, based on the proposed profile, and a fixed profile (always ON) can show the difference in the amount of the energy consumption as explained in Chapter4.

\subsection{Summary and Conclusions}

The proposed method in this research evaluates the occupancy behaviors and their potential impact on energy saving. A new sensor network method for real-time occupants and energy consumption monitoring was proposed where RTLS data were integrated with ZigBee energy meter data and provided information which was used for long-term simulation. The sensor networks provided basic tools for gathering the information on occupancy behavior and its interaction with IT equipment. 
UWB technology was selected in this research as an RTLS tool due to its high accuracy and ability to carry signals through doors and other obstacles; and ZigBee energy meters were chosen due to their low-cost and low-power wireless sensors. The basics of UWB technology and ZigBee energy meters, including components and operation details were discussed. Assessing the feasibility of this framework in real-world situation included an occupancy location and presence evaluation as well as IT equipment energy consumption evaluation in order to evaluate the energy saving potential.

The occupancy monitoring data gained from the UWB can be used to create the overall occupancy profile for each day of the week and probabilistic individual occupancy profiles for each of the occupants (weekdays and weekend days). Occupants' profiles demonstrate the occupants' hourly presence and absence in the office during week days and weekend days. Assuming that the occupants' pattern of using the space and IT equipment is constant throughout a certain period of time, these profiles can be used to estimate the long-term energy consumption. Then a simple procedure is proposed to calculate the time periods during which waste of energy occurred.

Finally, the estimation of the office energy consumption is carried out by a simulation software. One major key in estimating the energy consumption in the simulation process is its occupancy information. Two different types of profiles are created in accordance with the heating, cooling, lighting, and IT equipment energy consumption to assign the occupants information to the energy simulation model based on the overall occupancy profile and the probabilistic individual occupancy profiles. With the simulation model, the annual energy consumption can be calculated, as well as the difference between the estimated energy consumption based on the proposed occupancy profiles and fixed profiles. 


\section{CHAPTER 4 Implementation and Case Study}

\subsection{Introduction}

An experimental study with the objective of monitoring IT equipment energy consumption and occupancy behavior is proposed to evaluate the impact of occupancy behavior on IT equipment energy consumption in order to minimize energy consumption in office buildings. A test is carried out for one week of data acquisition in one of the research offices at Concordia University. UWB RTLS and ZigBee wireless energy meters are used for monitoring the occupancy behavior and IT equipment energy consumption, respectively. An UWB sensor cell, consisting of four sensors and four tags, is used to log the data. Four occupants are asked to participate in this test and wear slim tags during the test period. Simultaneously, energy meters are attached to the occupants' IT equipment to collect their energy consumption. The data from short-time monitoring are used to create daily and weekly occupancy profiles, overall occupancy profile and individual probabilistic occupancy profile.

The 3D model of the office is created in Revit. The gbXML format of the model is imported into IES $<\mathrm{VE}>$ software to run the simulation. Annual and weekly occupancy profiles based on the overall occupancy profile and individual probabilistic occupancy profile are created in the IES $<\mathrm{VE}>$ and assigned to the model. Besides, a fixed profile is chosen as the base line to compare the energy consumption of the proposed profile and the fixed profile. The comparison demonstrates significant energy consumption reduction regarding heating, cooling, lighting, and IT equipment energy consumption in the office. 


\subsection{Test Design}

A test is carried out for one week of data acquisition in one of the research offices at Concordia University. UWB RTLS technology is used for occupants monitoring. UWB provides the identity and location of occupants at short time intervals. Four occupants are asked to participate in this test and wear UWB slim tags during the test period. The layout of implementing UWB sensors is shown in Figure 4.1(a). A sensor cell, consisting of four sensors and four tags, is used to $\log$ the data. Four sensors were fixed at the four corners of the office ceiling and were connected with data and timing cables. The power of the sensors is supplied by a Power over Ethernet (PoE) switch. Timing cables are used to connect the sensors to synchronize the signals from a tag to different sensors. The sensors are calibrated using a tag as a reference point with a known position. The solid lines show the data cables connecting the sensors with the PoE switch, whereas the dotted lines show the timing cables.

On the other hand, ZigBee wireless energy meters are used for monitoring the energy consumption of IT equipment (i.e., monitors, computers, and printers), which provides the instantaneous and cumulative IT equipment energy consumption data simultaneously. Energy meters should be attached to the IT equipment in the office which is in use by the occupants. Figure 4.1(b) shows the layout of implementing the ZigBee network. The wireless ZigBee network includes a gateway and nine energy meters. There are connectivity problems in the ZigBee wireless network. The connection between energy meters and gateway was lost several times during the test period. Therefore, the period of the test was extended. 


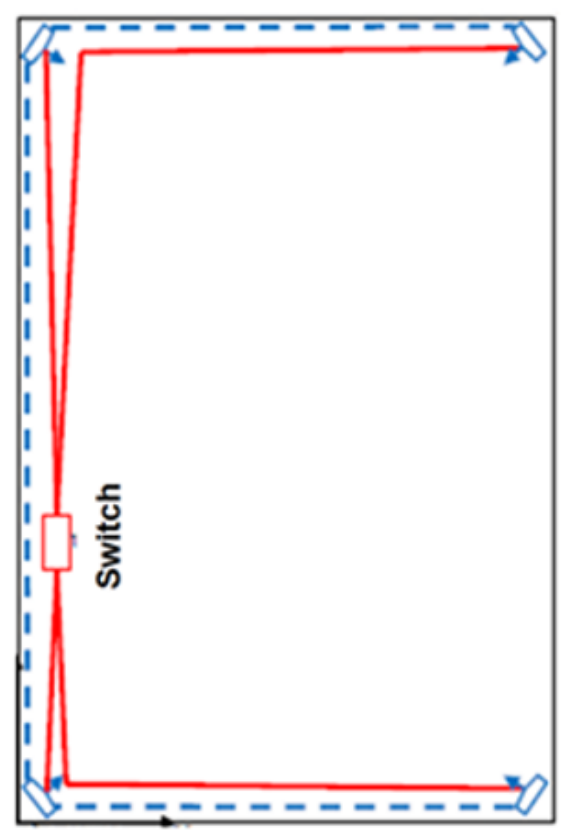

(a) Distribution of UWB sensors

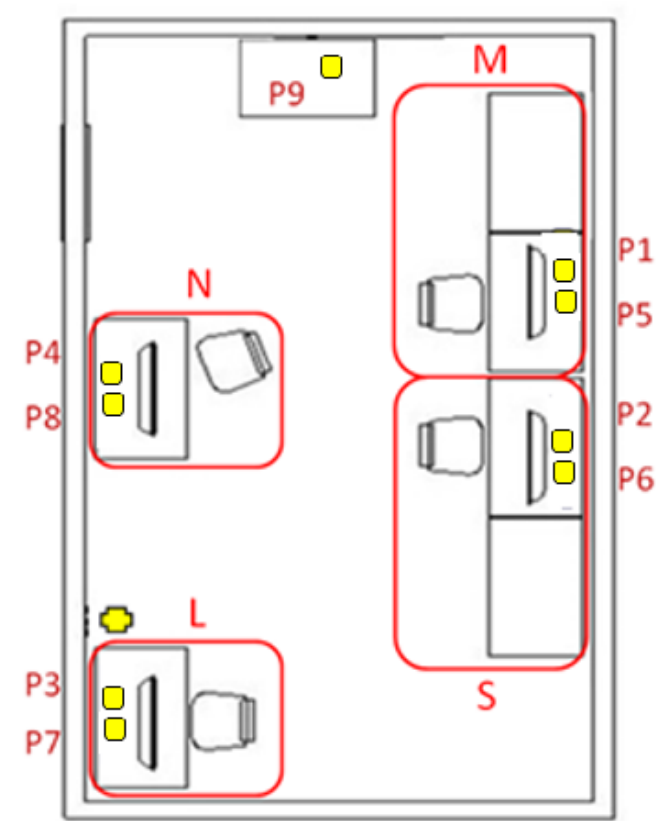

(b) Distribution of ZigBee energy meters

$\square$ Sensor — Timing cable —Data cable 凸 ZigBee Gateway $\square$ ZigBee energy meter

Figure 4.1 Layout of implementation of wireless sensor networks

In this test, the update rates for UWB and ZigBee energy meters are set in accordance to each other. Four UWB tags with an individual update rate of two readings per minute are registered in the UWB logger application. Simultaneously, energy meters attached to the occupants' IT equipment were set with an individual update rate of about two readings per minute in average. The specifications of the IT equipment are shown in Table 4.1.

Table 4.1 IT equipment specifications

\begin{tabular}{|c|c|c|}
\hline Equipment & Specifications & Energy meters \\
\hline \multirow{4}{*}{ Computers } & Dell Precision T3500 & P1 \\
\cline { 2 - 3 } & Dell AS501 & P2 \\
\cline { 2 - 3 } & Dell Precision T3500 & P3 \\
\cline { 2 - 3 } & HP Z210 & P4 \\
\hline \multirow{4}{*}{ Monitors } & Dell AX510, 23 inch & P5 \\
\cline { 2 - 3 } & Dell AS501, 24 inch & P6 \\
\cline { 2 - 3 } & IBM 9494, 18.1 inch & P8 \\
\cline { 2 - 3 } & Dell1908FPc, 19 inch & P9 \\
\hline
\end{tabular}




\subsection{UWB and Energy Meters Raw Data}

As shown in Figure 4.2, occupants' 2D location and time data are logged during the test including the UWB tag ID, coordinates, date, and time in each of the readings.

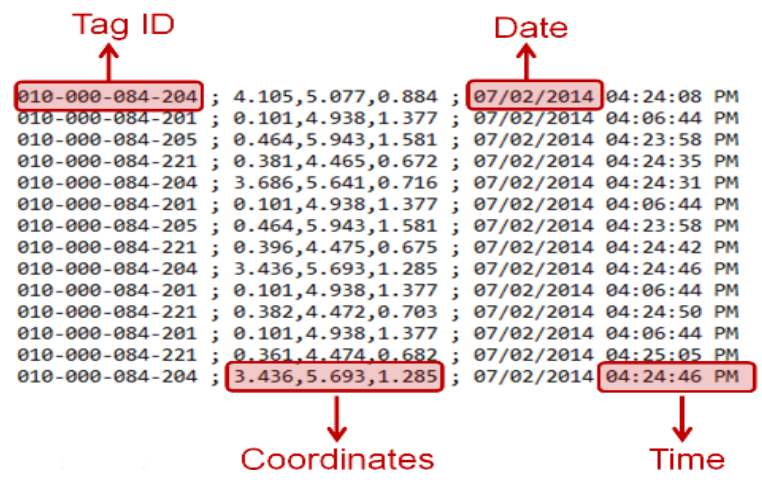

Figure 4.2 Sample location raw data

Figure 4.3 shows a sample of ZigBee energy meters raw data including the device ID, date and time, instantaneous and cumulative energy consumption.

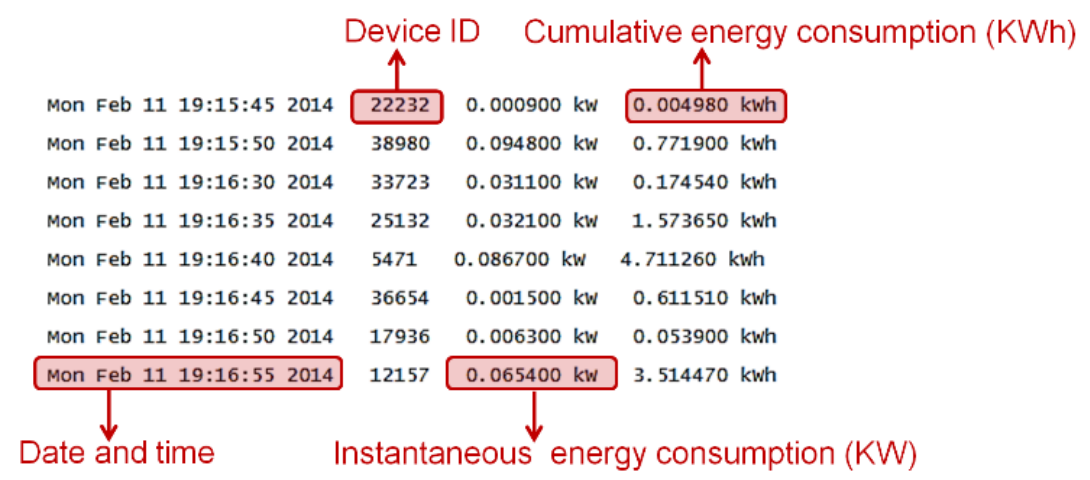

Figure 4.3 Sample ZigBee energy meters raw data

\subsection{Data Processing and Visualization}

The ZigBee energy meters and UWB data must be separately processed to provide the location and energy measurement data, respectively. The data can be fused to understand the occupants' energy consumption patterns. In this test, the logger applications gather location and the IT 
equipment energy consumption data into CV-based Excel sheets. Furthermore, some processing is required to provide the UWB and ZigBee energy meters datasets which are aligned in terms of time such as: data synchronization, averaging (one reading per minute), and filtering of false readings. This processing assures providing reliable data for the analysis. Since a huge amount of information is saved in Excel files, to improve the efficiency and convenience, Visual Basic for Application (VBA) is used. VBA is an effective tool to automate the frequently used repetitive calculations. The advantage of using VBA for data processing and visualization of test data is that it provides a tool to automatically generate customized reports, charts (occupancy charts, occupancy maps, and energy consumption charts).

\subsubsection{Occupancy Maps}

A worksheet is developed in Excel to analyze and visualize the collected data. Occupancy maps are created daily to show occupants' locations in the office based on UWB coordinates (X, Y). As shown in Figure 4.4, each symbol indicates an occupant (i.e. S, M, L, and N) and each point indicates the location of a specific occupant at a certain time (i.e. every minute). The distribution of occupants' locations in each map demonstrates the occupants' presence and locations on every day of the week. For example, all occupants came to the office during weekdays except occupant S who was absent on Monday. During the weekends, occupant L did not come to the office while occupant $\mathrm{N}$ came on both days and occupant M and S came on Saturday and Sunday, respectively. The density of each occupant in a location shows the length of time that he/she was in the office. However, these occupancy maps do not determine the time at which each occupant was in the office. 

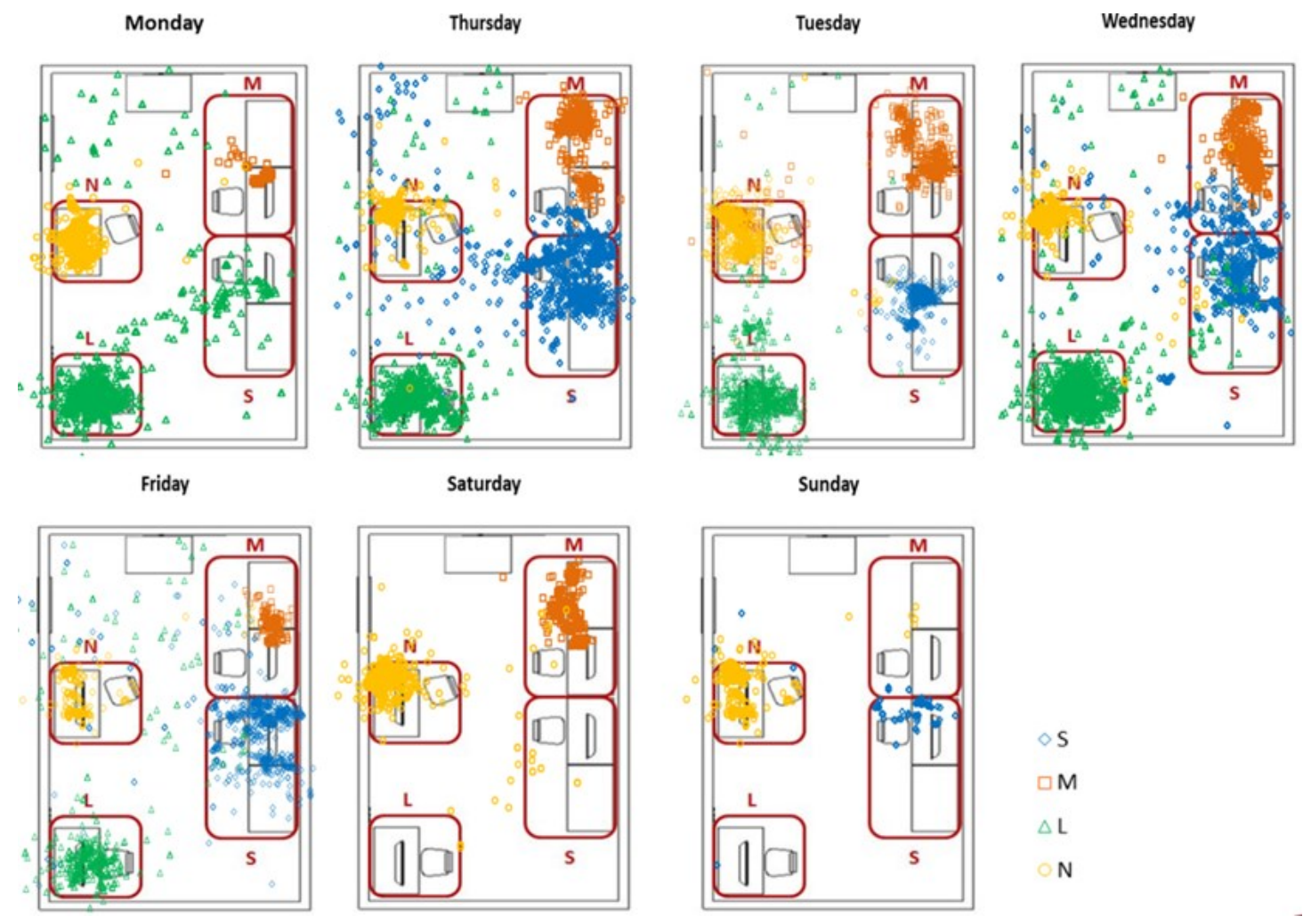

Figure 4.4 Occupancy maps

\subsubsection{Occupancy Charts}

Occupancy charts are developed to illustrate occupants' daily presence as shown in Figure 4.5. The charts show that the occupants left the office several times during the day and sometimes they only came to the office for a few minutes (e.g. occupant L at around 6:00 PM on Monday). Moreover, they had different daily presence patterns. For example, occupant $\mathrm{S}$ came to the office at around noon and was the last one who left the office on Friday. On the other hand, on weekend days, only two occupants came to the office and stayed for a short period of time. Occupant $\mathrm{N}$ came to the office every day and stayed more time at the office compared to others. 


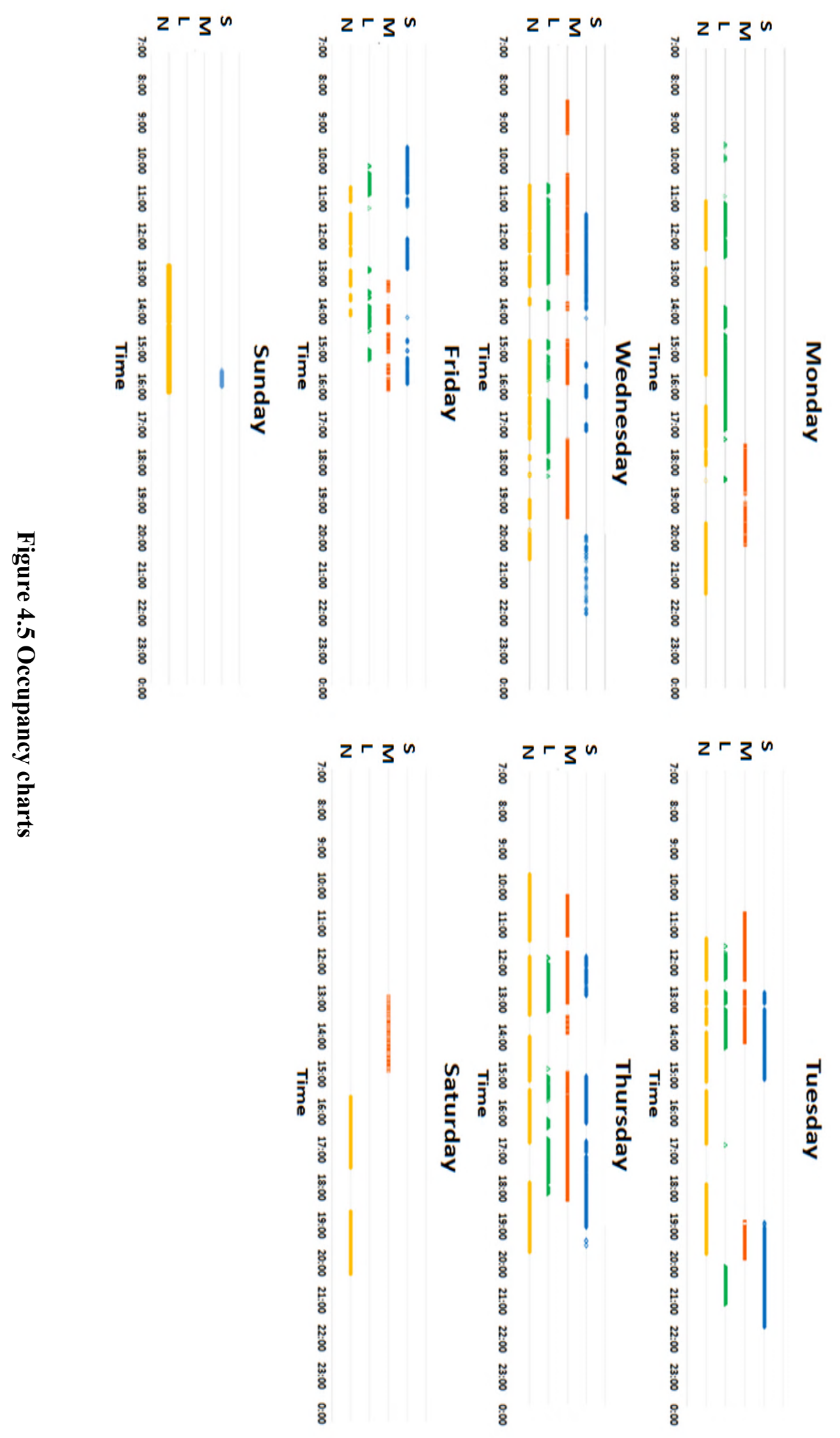




\subsubsection{Zigbee Energy Meters Data Processing}

Figure 4.6 shows daily electricity consumption for different monitors for different days of the week. The horizontal axis represents the time, whilst the vertical axis illustrates the cumulative energy consumption for monitors.
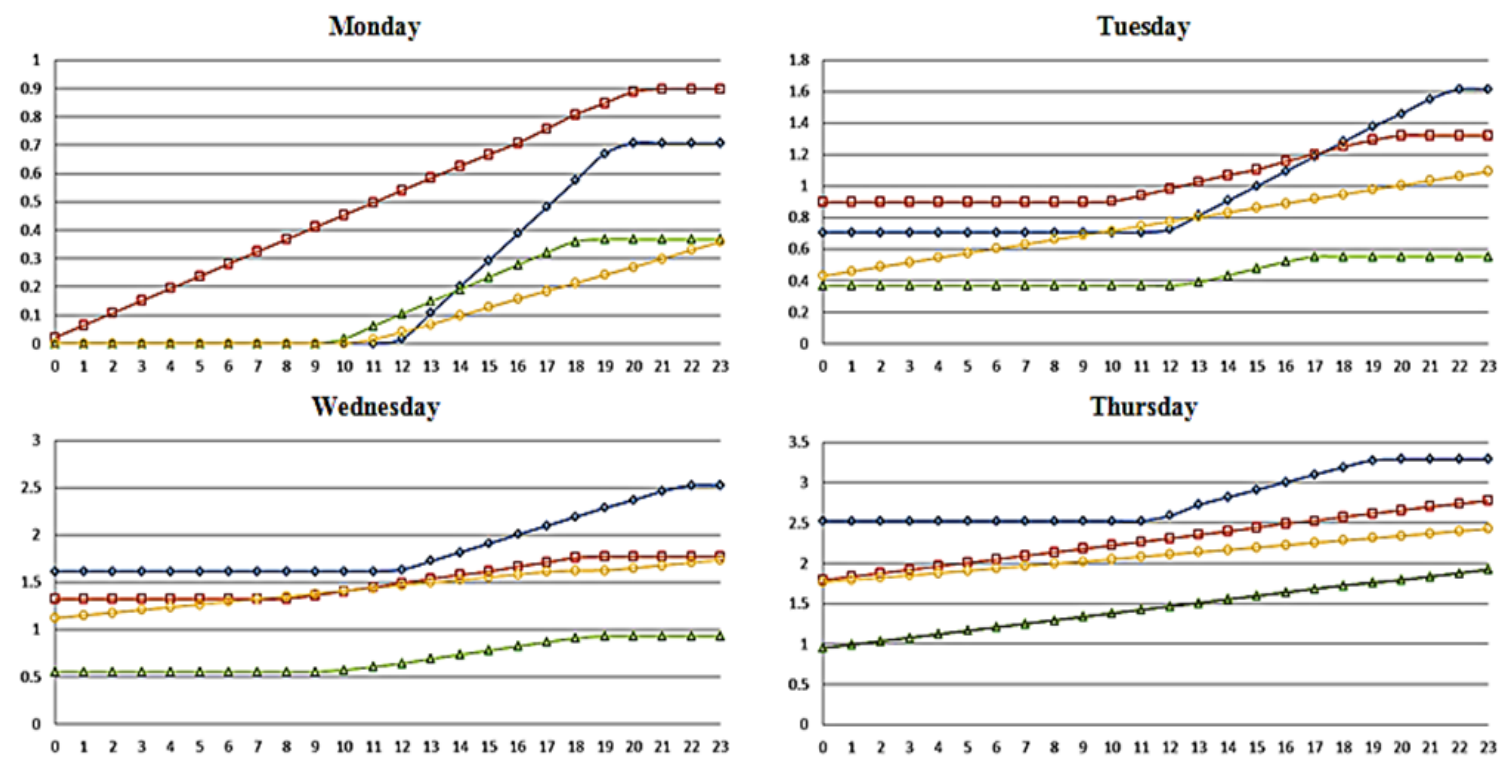

Thursday

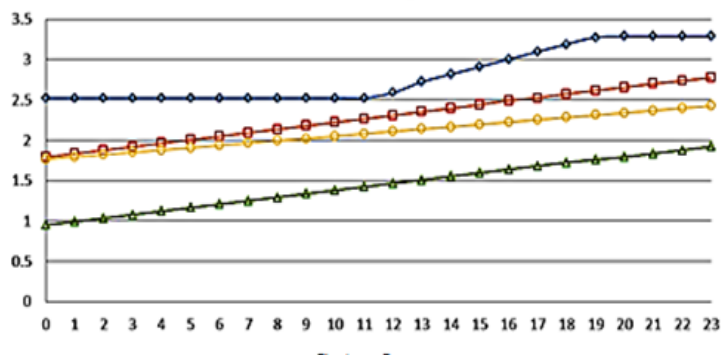

Friday
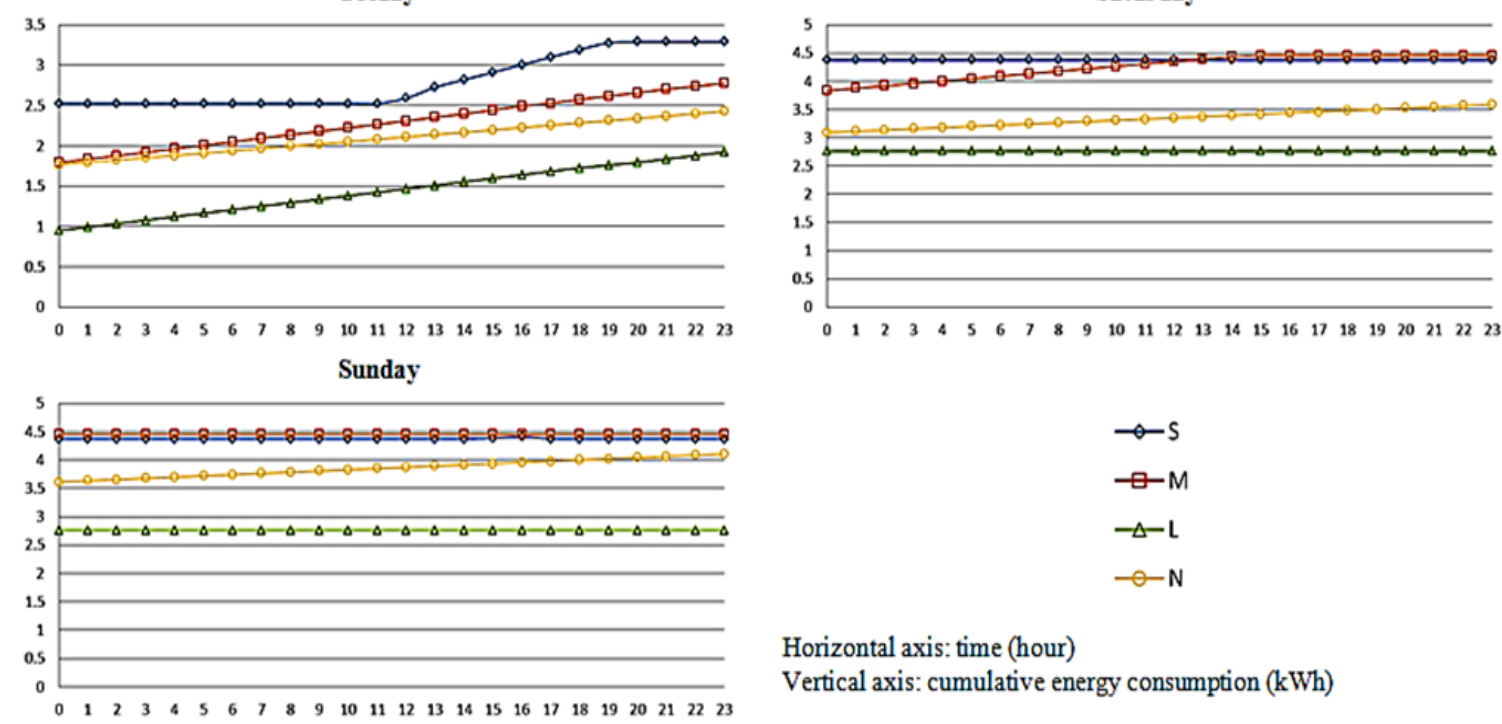

Horizontal axis: time (hour)

Vertical axis: cumulative energy consumption (kWh)

Figure 4.6 Energy consumption of monitors 
The slope of the curve illustrates the energy consumption for each monitor; the steeper the slop, the greater the rate of energy consumption. Analyzing energy consumption data shows that computers and monitors with different specifications consumed energy differently. The size and brand of the monitors would impact their energy consumption.

Comparing the information in Table 4.1 with the measured energy consumption demonstrates that monitors with bigger screens consumed more energy than the ones with smaller screens. For instance in Figure 4.6, it is shown that the 19" monitor of occupant N consumed approximately $60 \%$ less energy than the 24 " monitor of occupant S. However, although occupant L's monitor is smaller than that of the occupant $\mathrm{N}$, it consumed more energy because it is an old monitor. Another point that can be obtained from the graphs is that occupant $\mathrm{N}$ never turned off her monitor during the test.

Moreover, monitors energy consumption varied regarding the percentage of brightness (i.e. $100 \%, 70 \%, 50 \%, 20 \%$, and $10 \%$.) as shown in Figure 4.7 .

Similar graphs were created for energy consumption for computers. Comparing the slops in Figure 4.8 indicates that the computers for occupants $\mathrm{S}, \mathrm{M}$ and $\mathrm{L}$ had almost the same energy consumption whilst occupant N's computer, which is a newer system, consumed less.

Focusing on the graph corresponding to Saturday and Sunday shows that occupant L turned off his computer during the weekend while the other computers were on. Moreover, Figure 4.9 shows the instantaneous energy consumption of the printer. As it is shown in this figure, there are significant rises in printer's energy consumption between 17:25 and 17:55, which were due to occupants' printing papers. 


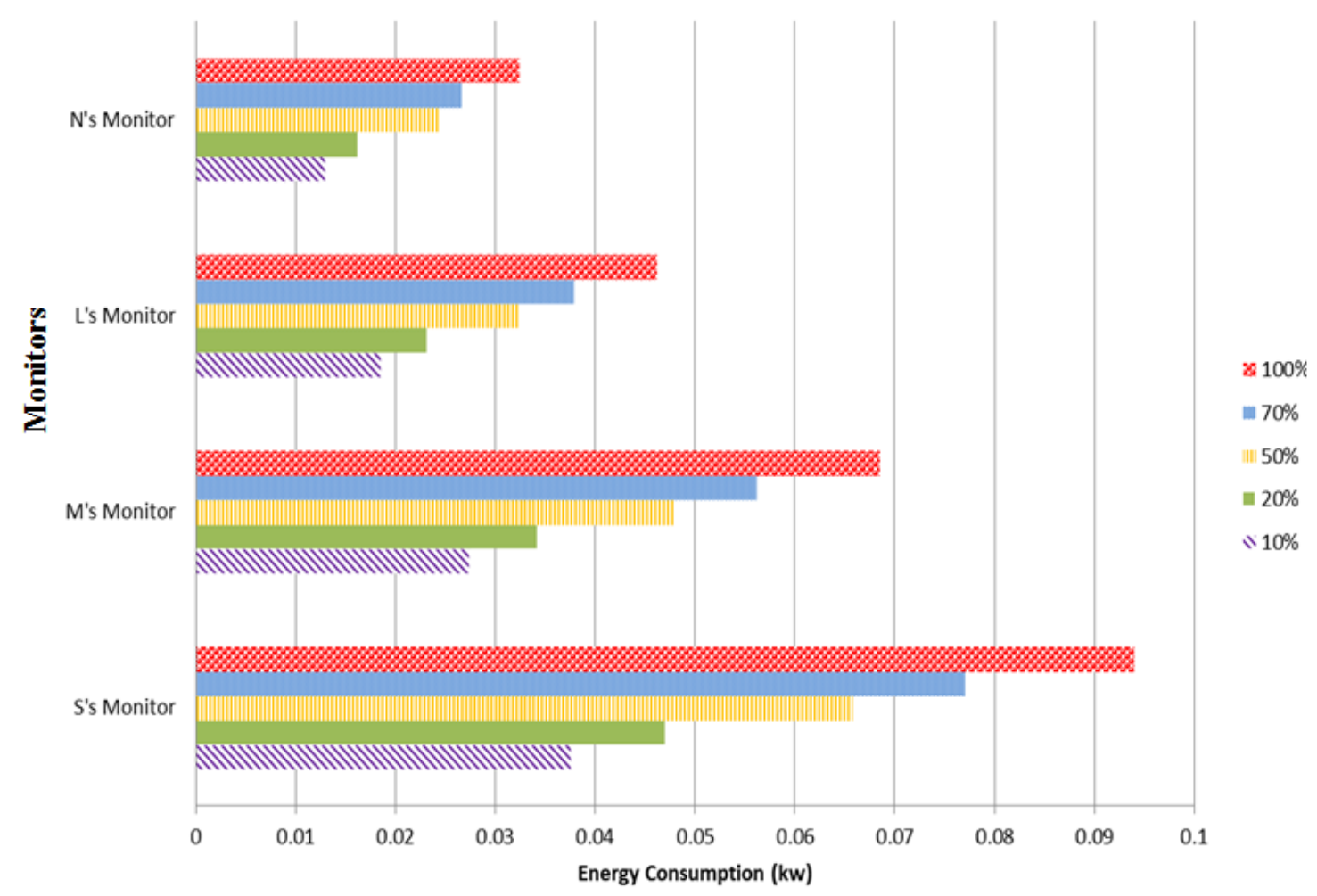

Figure 4.7 Monitors energy consumption varied regarding the percentage of brightness

\subsection{Visual Analysis of Collected data}

The Ubisense Location Platform supports spatial event detection that detects objects and turning location events into data to be used by applications and visualization including $2 \mathrm{D}$ and $3 \mathrm{D}$. The Ubisense logger application and the Smartenit logger are installed on a machine to log the UWB RTLS and the ZigBee energy meters data during the test.

These loggers gather the data in CV-based text files that can be open as Excel sheets. Data fusion is implemented in Excel. As explained in Section 3.4.4, the location and energy meter data sets must be pre-processed to be fused in order to preprocess the data.

Some collected values are outside the expected range due to systematic errors which are filtered out from the logged data. Besides, there are missing readings during data collection which are 
filled by data interpolation. Then the average value for each data collection step is calculated to synchronize the UWB and ZigBee readings, i.e., one reading per minute.
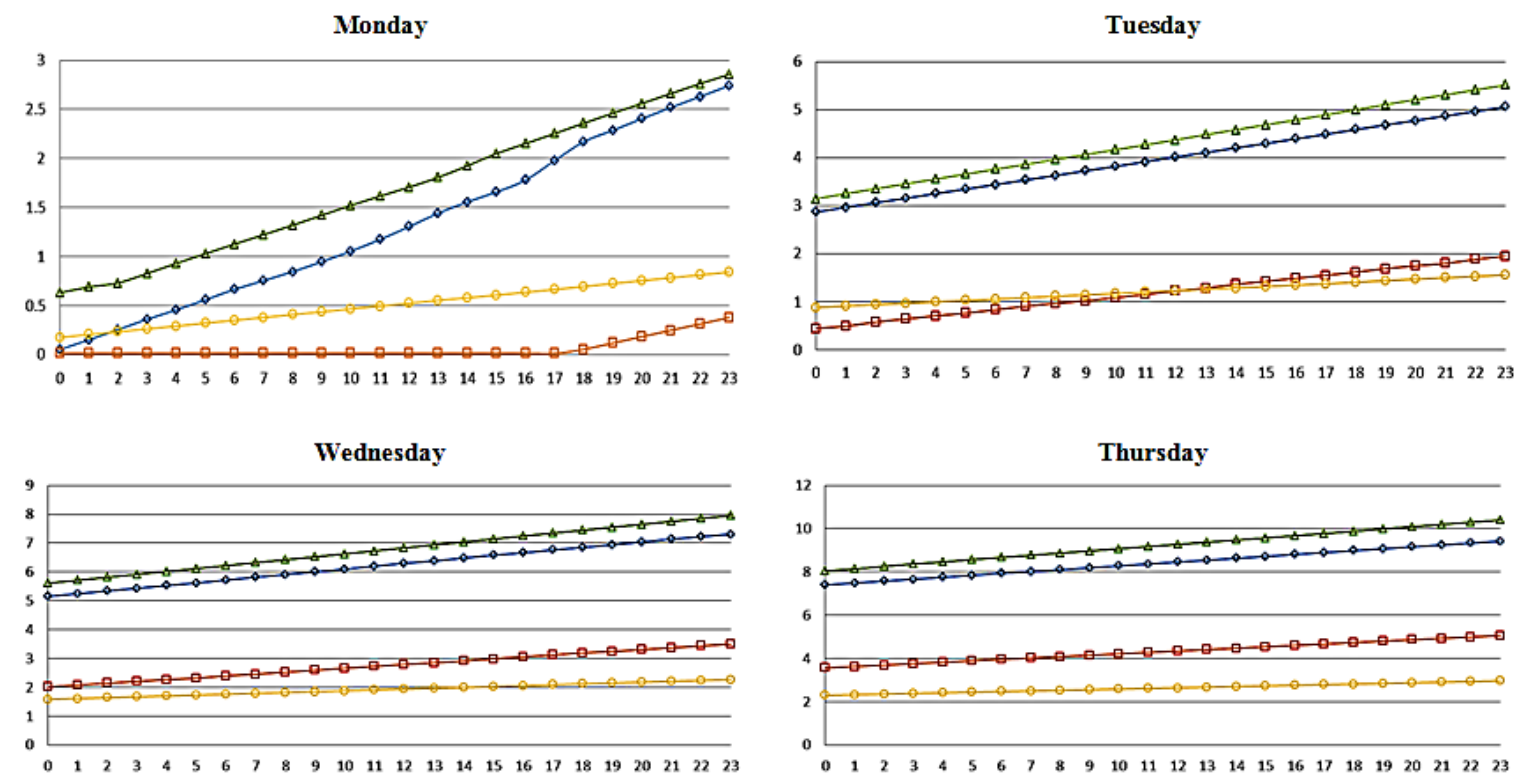

Friday
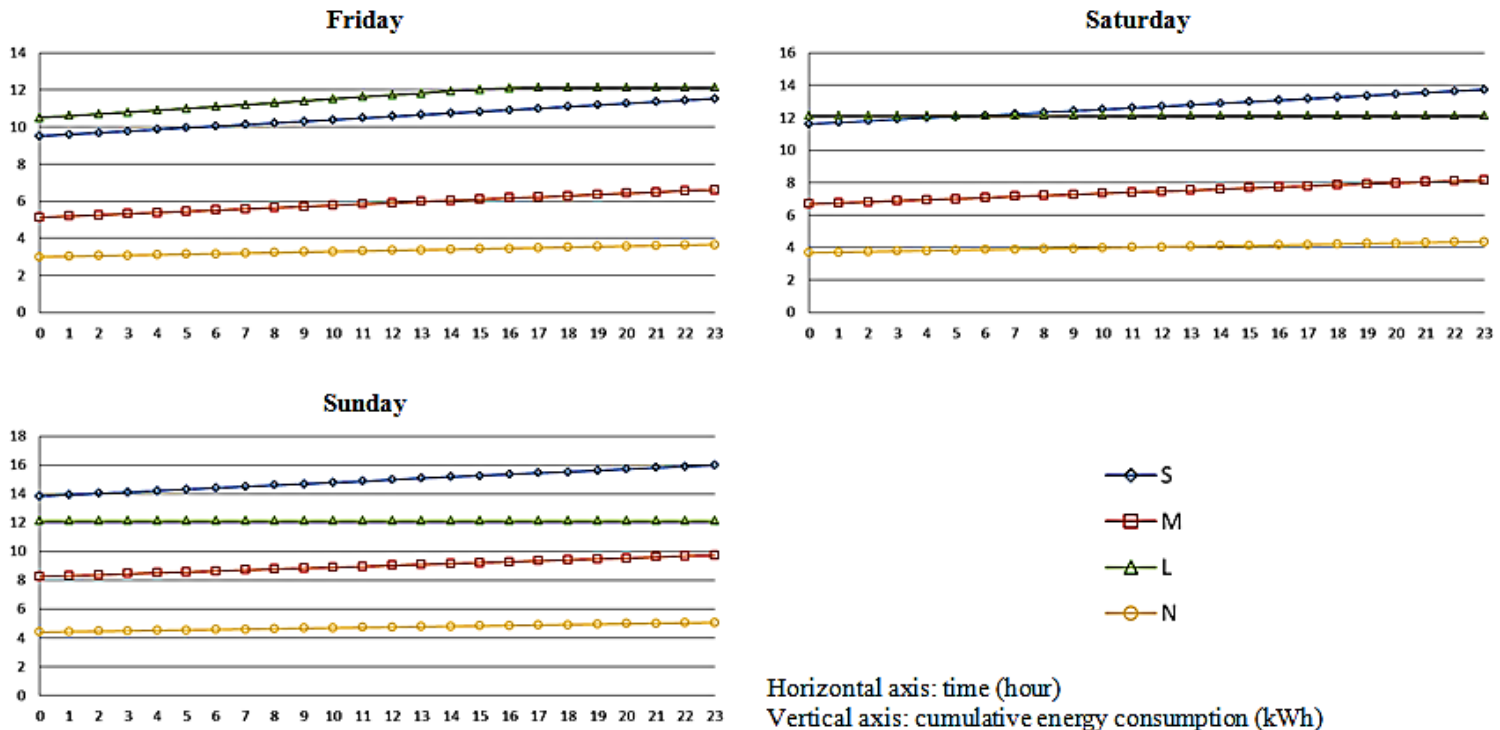

$$
\begin{aligned}
& \rightarrow S \\
& \square-M \\
& \triangle-L \\
& -N
\end{aligned}
$$

Horizontal axis: time (hour)

Vertical axis: cumulative energy consumption (kWh)

\section{Figure 4.8 Energy consumption of computers}




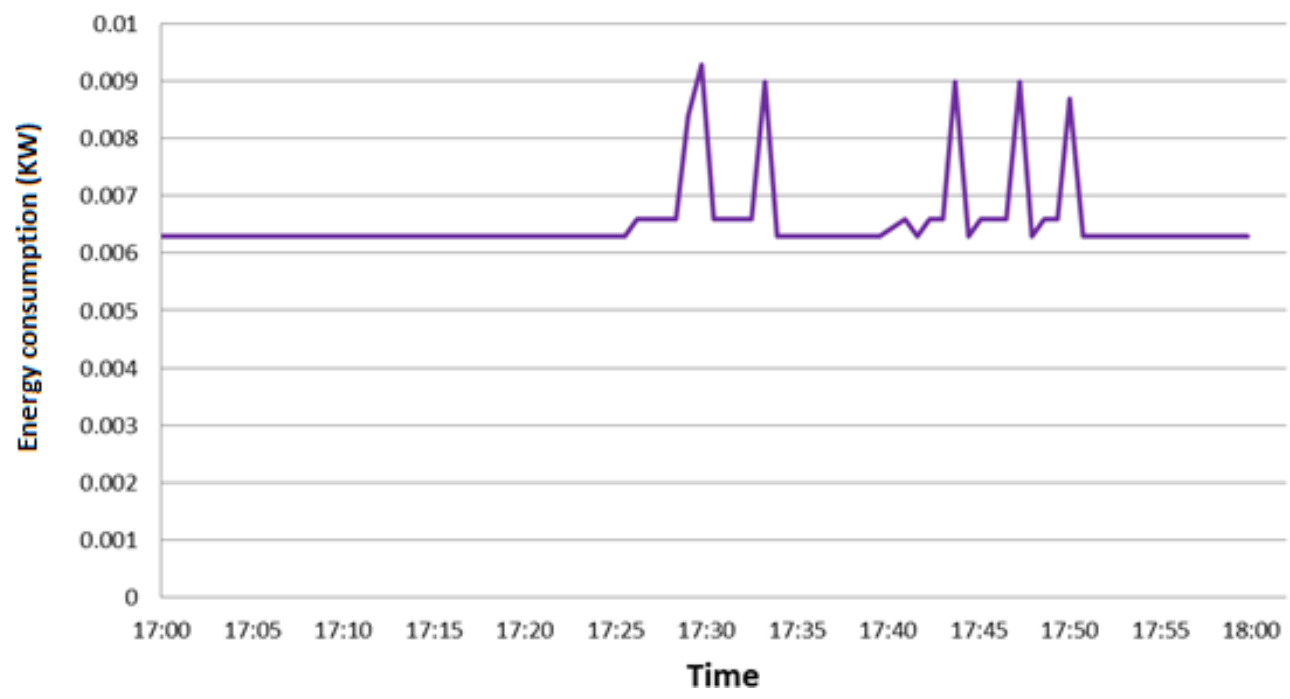

Figure 4.9 Energy consumption of printer

Monday was selected as a sample for the observations. Regarding the occupancy chart, Figure 4.5, occupants L, N, and $\mathrm{M}$ entered the office at around 9:30 AM, 11:00 AM, and 5:00 PM, respectively.

It seems that L left the office from 1:00 to 2:00 PM for lunch while $\mathrm{N}$ went for lunch at 12:30 PM and came back at 1:00 PM. L left the office in the evening at 7:00 PM. N left at around 10:00 PM and M stayed in the office for about four hours. Observing the energy consumption chart for monitors, Figure 4.6, shows that L's monitor has been turned on at about 10:00 AM, after the time he came to the office, and was on until 7:00 PM. Moreover, regarding the energy consumption chart for computers in Figure 4.8, L's computer was on all day long. S's monitor was on from noon until about 8:00 PM resulting in a rise in his monitor energy consumption. While his computer was on all day long, he was not in the office on Monday. The occupancy map in Figure 4.4 shows that L was in S's zone for a period of time and probably was using his equipment which resulted in increasing its energy consumption. 


\subsection{Data Fusion for Calculating Energy Waste}

Results from the test demonstrate that there are two different types of behavior regarding saving energy in the office: (1) turning off IT equipment when not in use (user not in zone or out of the office even for a short time), which results in reducing the energy consumption; and (2) leaving IT equipment on all days long. For example, as shown in Figure 4.6, occupant $\mathrm{S}$ turned off his monitor before his leaving almost every day, while occupant $\mathrm{N}$ never turned her monitor off. In order to calculate the waste of energy related to occupants' behavior, we chose occupant L behavior on Monday. As explained in Section 3.4.4, in order to calculate the total waste of energy during this day, first, the time at which $\mathrm{L}$ was in the office has been calculated $\left(\mathrm{T}_{1}=291\right.$ $\mathrm{m})$. During this period, he spent $\mathrm{T}_{3}=243 \mathrm{~m}$ in his zone and $\mathrm{T}_{4}=48 \mathrm{~m}$ out of his zone. He turned his monitor on once he arrived to the office and turned it off when he left the office. However, his computer was running all day long. Therefore, during the time he was in the office and he was in his zone, his monitor and computer were on for a period of $\mathrm{T}_{7}=48 \mathrm{~m}$. Moreover, his equipment was running during the time he was not in the office between 9:35 AM and 6:38 PM. In addition, his computer was running even after he left the office till next day. Table 4.2 shows the summary of the waste of energy calculation for one day. In conclusion, it is estimated that if the occupant L's monitor and the computer were turned off during the time that he was not in the office or in his zone, about $0.38 \mathrm{KWh}(20 \%)$ and $6.58 \mathrm{KWh}(83 \%)$ energy could have been saved on that day, respectively. Calculated results in Table 4.2 demonstrate that applying a fixed schedule to run IT equipment in institutional buildings in which there are several research offices and computer labs will result in a significant waste of energy over long time especially during weekend days, holidays, and summer breaks. Therefore, dynamic occupancy driven profiles would reduce the energy consumption. 
Table 4.2 Time and KWh waste of energy

\begin{tabular}{|c|c|c|c|c|c|c|c|c|}
\hline & \multicolumn{4}{|c|}{ Occupant in the office (min) } & \multirow{2}{*}{\multicolumn{2}{|c|}{$\begin{array}{l}\text { Occupant not in } \\
\text { the office ( } \mathrm{min})\end{array}$}} & \multirow{3}{*}{$\begin{array}{c}\text { Total time of waste } \\
\text { of energy } \\
\text { (min) }\end{array}$} & \multirow{3}{*}{$\begin{array}{l}\text { waste of } \\
\text { energy } \\
\text { (KWh) }\end{array}$} \\
\hline & \multicolumn{2}{|c|}{$\begin{array}{l}\text { Occupant in } \\
\text { zone }\end{array}$} & \multicolumn{2}{|c|}{$\begin{array}{l}\text { Occupant not } \\
\text { in zone }\end{array}$} & & & & \\
\hline & $\begin{array}{l}\text { Eq. } \\
\text { ON }\end{array}$ & $\begin{array}{l}\text { Eq. } \\
\text { OFF }\end{array}$ & $\begin{array}{l}\text { Eq. } \\
\text { ON }\end{array}$ & $\begin{array}{l}\text { Eq. } \\
\text { OFF }\end{array}$ & Eq. ON & $\begin{array}{l}\text { Eq. } \\
\text { OFF }\end{array}$ & & \\
\hline Monitor & 243 & 0 & 48 & 0 & 246 & 903 & $48+246=294$ & 0.38 \\
\hline Computer & 243 & 0 & 48 & 0 & 1149 & 0 & $1149+48=1197$ & 6.58 \\
\hline
\end{tabular}

\subsection{Statistical Model of Occupancy Profiles}

As explained in Section 3.4.3, the data obtained from the UWB occupancy monitoring are used to create daily and weekly user's profiles. Figure 4.10 shows the overall hourly occupancy presence in the office during a week for all occupants.
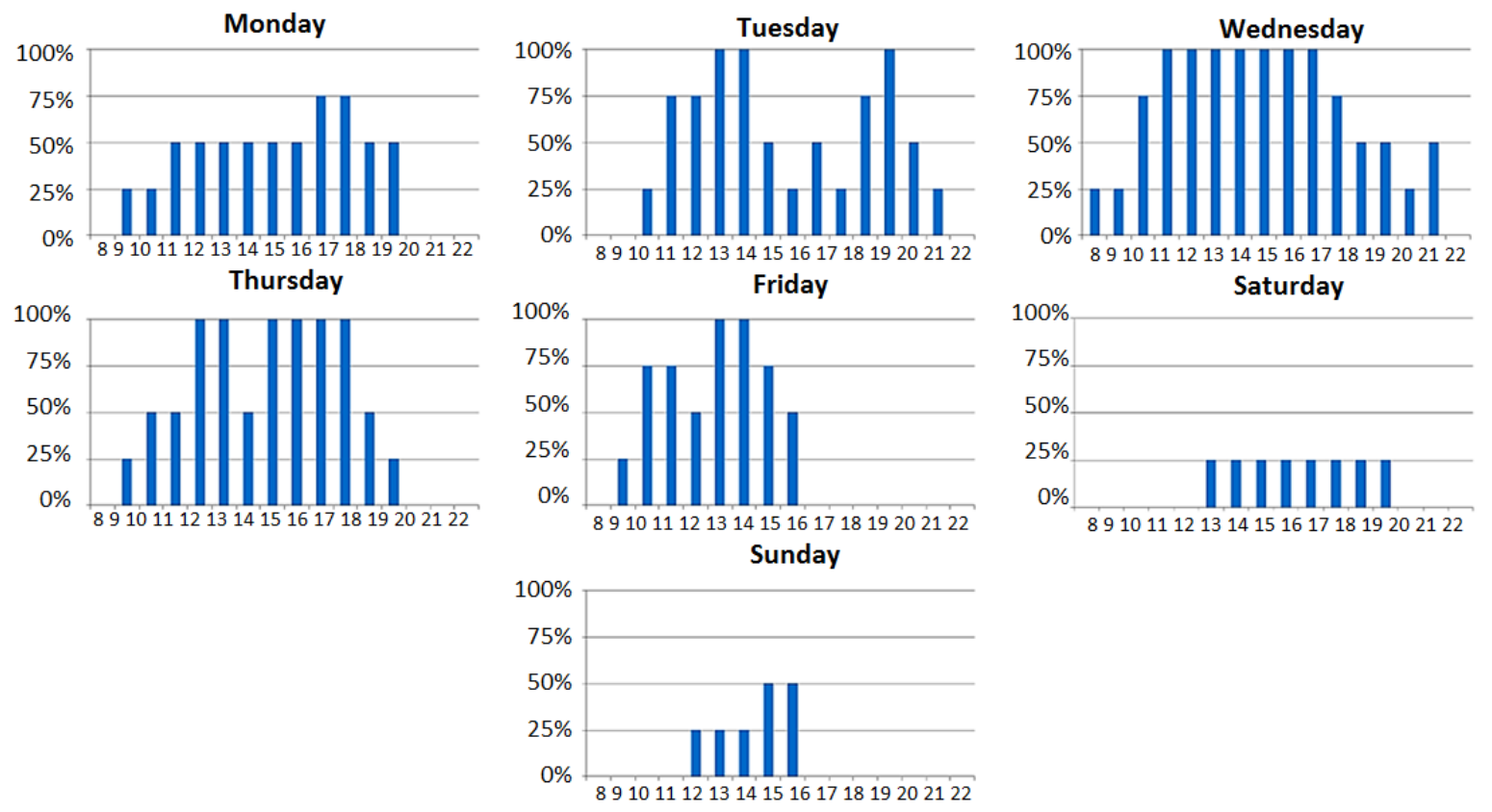

Figure 4.10 Overall occupancy profiles

For example, a value of $100 \%$ at 10:00 AM on Wednesday means that all the occupants are expected to be in the office during that hour. As expected, there is a decrease in the number of occupants during the weekend. 
Figures 4.11(a) and 4.11(b) show the histograms of probabilistic individual occupancy profiles for each occupant during the weekdays and weekend days, respectively. For example there is $20 \%$ probability to find user $\mathrm{S}$ in the office at 10:00 AM on weekdays.

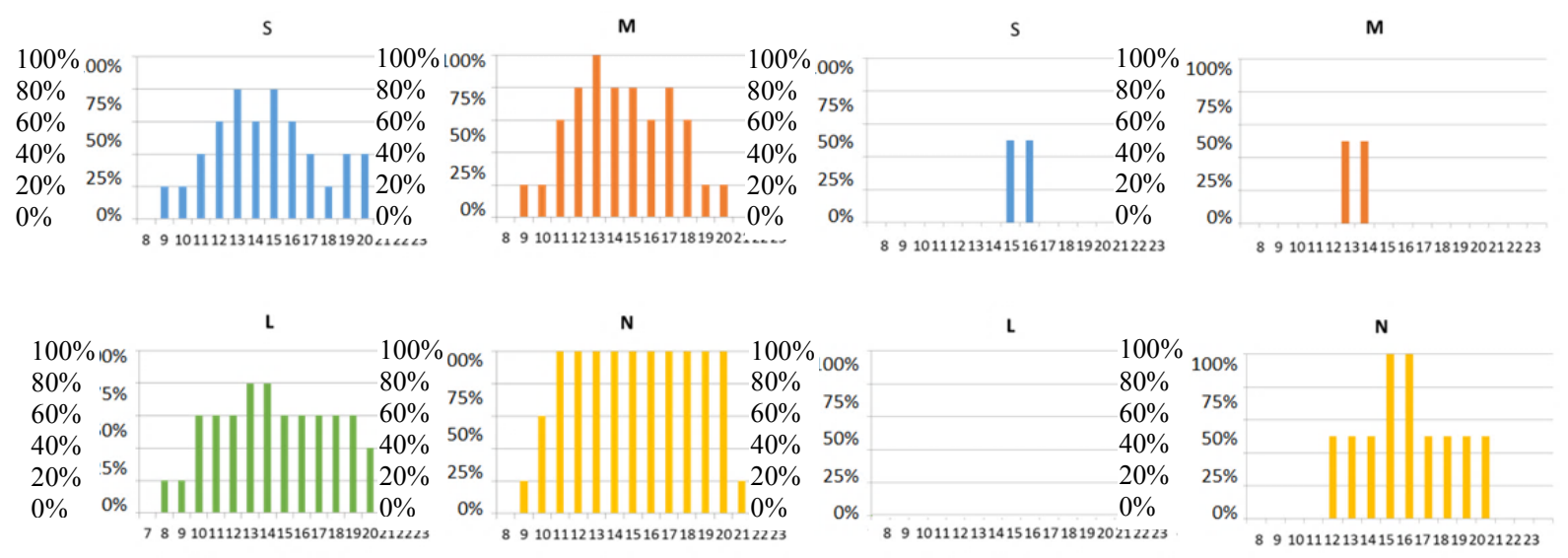
(a) weekdays
(b) weekend days

Figure 4.11 Probabilistic individual occupancy profiles

\subsection{Energy Consumption Simulation}

The test bed is located on the $9^{\text {th }}$ floor of the Concordia University EV building. Therefore, only the $3 \mathrm{D}$ model of that part of the building is created in the Revit software including the neighboring rooms and spaces shown in Figure 4.12.

\subsubsection{Heating and Cooling Profile}

As mentioned in Section 4.2, the test is carried out in a multi-occupants office. Therefore, it is assumed that when any occupant enters the office, the heating and cooling system should start. It means that there are only two states for the heating and cooling, ON or OFF. Occupancy profiles, as explained in Sections 3.4.3 and 4.7, are created based on occupants' presence gathered by UWB. The occupancy profiles show the number of occupants and their identification 
in the office. The 3D of the model is created in Revit and is exported into IES $<$ VE $>$ software with the gbXML format to run the simulation.

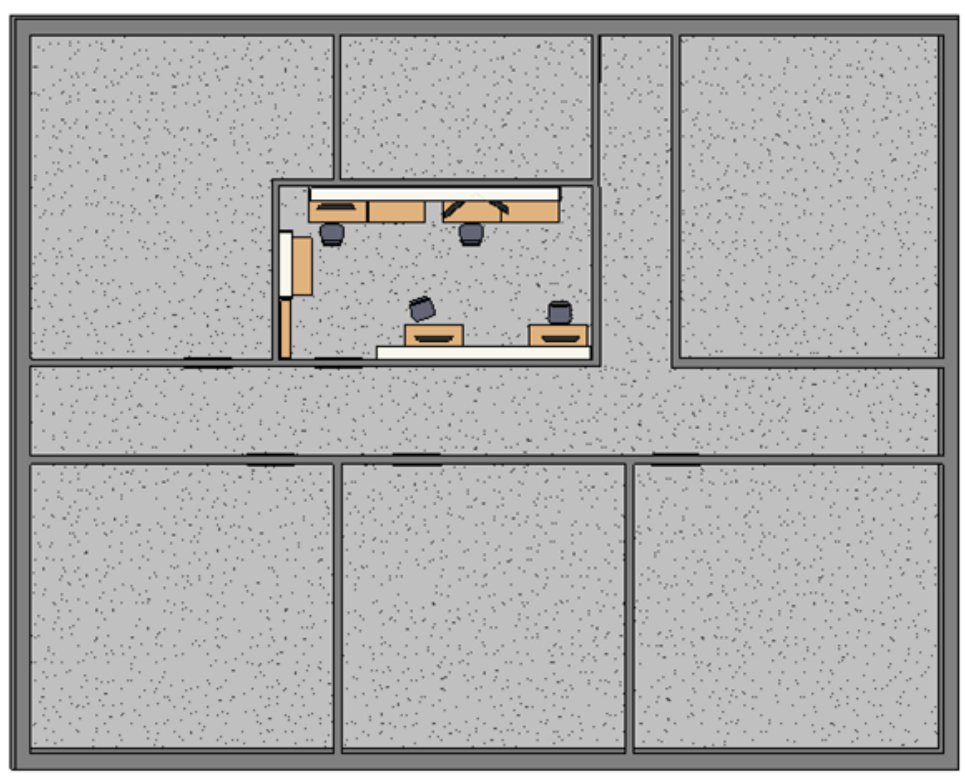

Figure 4.12 3D model of the test bed created in Revit

Based on the overall occupancy profile, daily profiles are defined as series of values applied at different times of weekdays and weekend days on an hourly basis. For instance, the number of occupants in the office at 8:00 AM on Monday was zero. While on the same day at 4:00 PM there were two occupants in the office. Therefore, the values of zero and one are assigned to these two hours of the day, respectively. If there was at least one occupant in the office, it means that $100 \%$ of the maximum value of the heating and cooling is demanded; and if there was no occupant in the office, it means that $0 \%$ of the maximum value of the heating and cooling is needed. Figure 4.13 demonstrates the heating and cooling profile which is assigned to Thursday based on the collected data during the test period. 


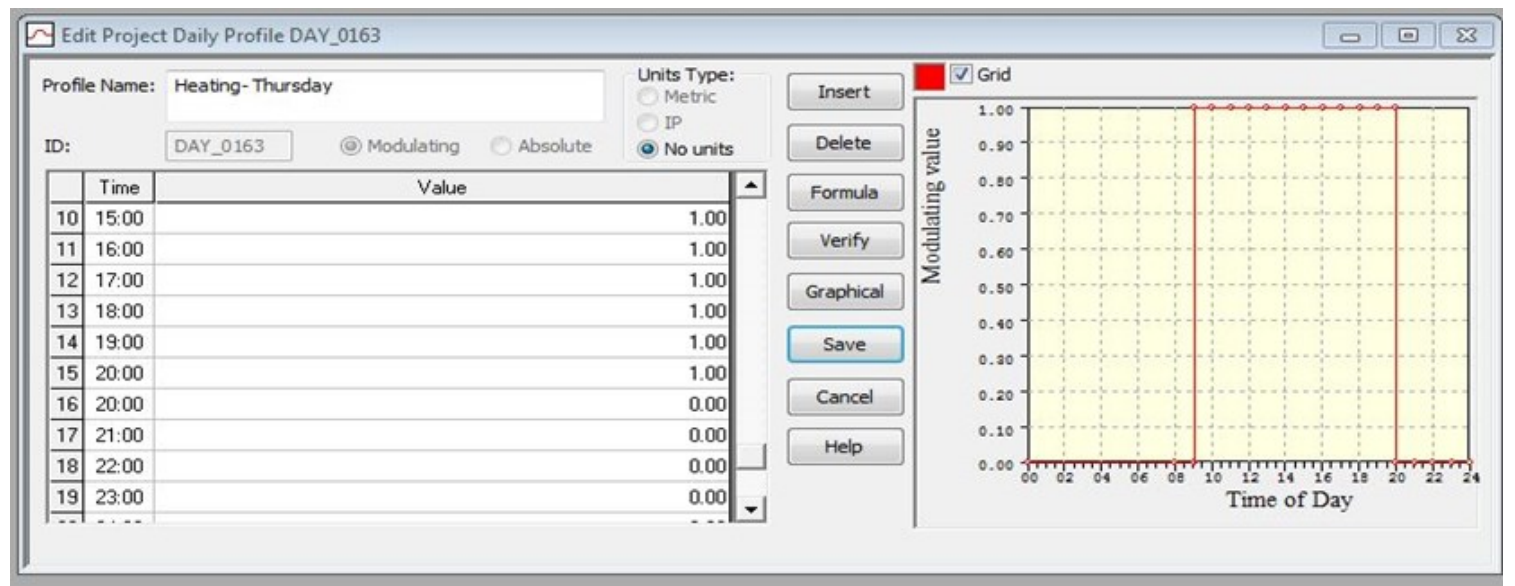

Figure 4.13 Thursday heating and cooling profile

On Thursday, the first occupant came to the office starting around 9:00 AM therefore the heating and cooling system started working. The last occupant left the office at around 8:00 PM and at that time the heating and cooling system would stop running. Similar to Thursday, in accordance with the overall occupancy profile shown in Figure 4.10, the daily profiles are applied to weekdays and weekend days.

Figure 4.14 shows the profiles created for each day of the week in IES $<\mathrm{VE}>$. Annual and weekly profiles are the only two types of profiles which can be applied to the model in this software. In this regard, to be able to simulate the annual energy consumption based on the monitored data, first daily profiles are created.

Then, these daily profiles are assigned to each day of the week to generate the weekly profile. This proposed heating and cooling weekly profile is assigned to the heating and cooling section in the software to run the annual heating and cooling energy consumption simulation. The office heating and cooling energy consumption based on the overall occupancy profile is shown in Figure 4.15. 

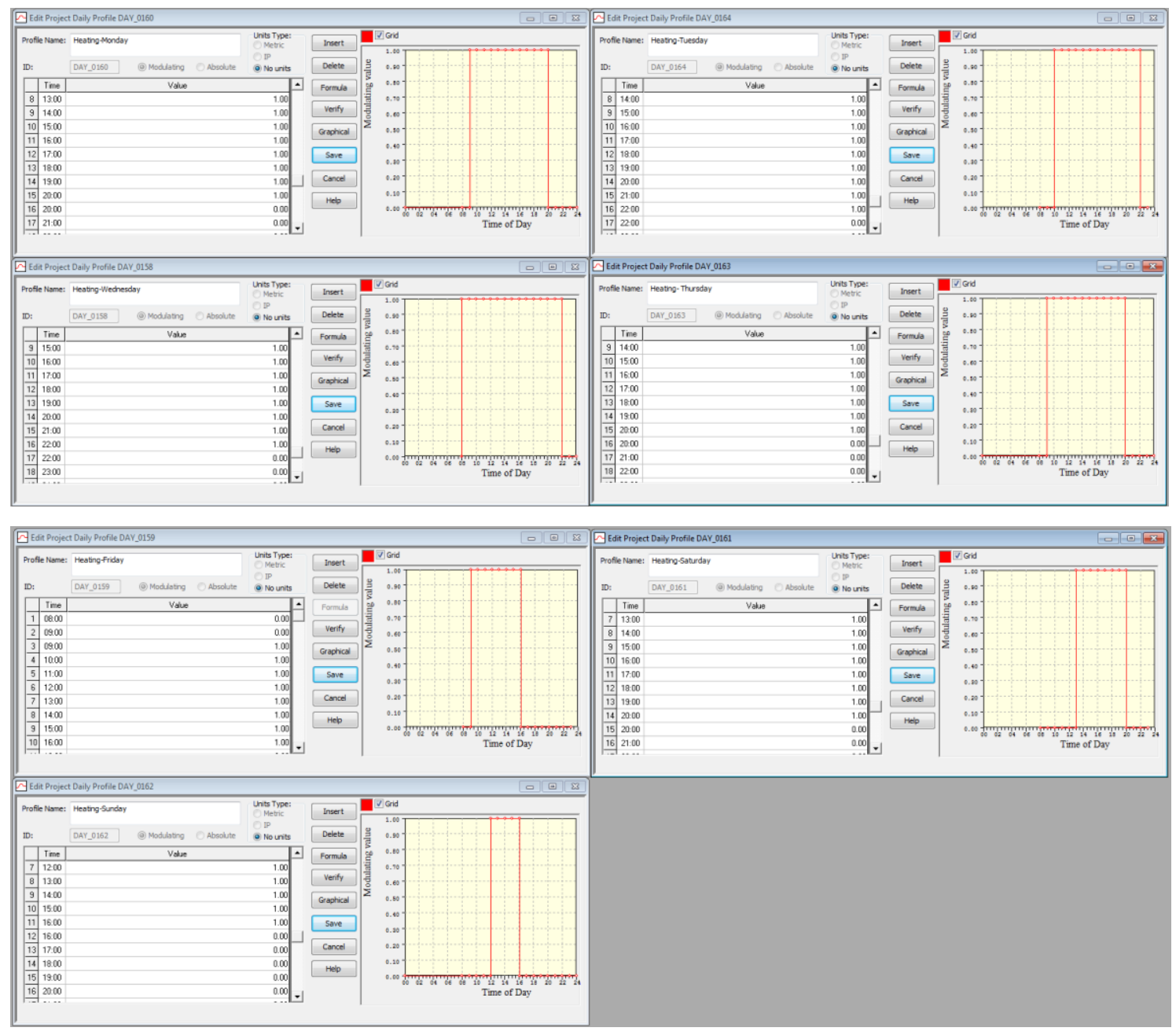

Figure 4.14 Weekly heating and cooling profile proposed based on monitored data

It has been tried to enter inputs similar to the real office building (i.e. building envelope elements, heating and cooling set points, and location weather data) to improve the accuracy of the simulation of the heating and cooling loads. However, as shown in Appendix C, more detailed information about HVAC system (i.e. boilers and chillers power consumption and their efficiency) is required to obtain more accurate results for the heating and cooling loads. 


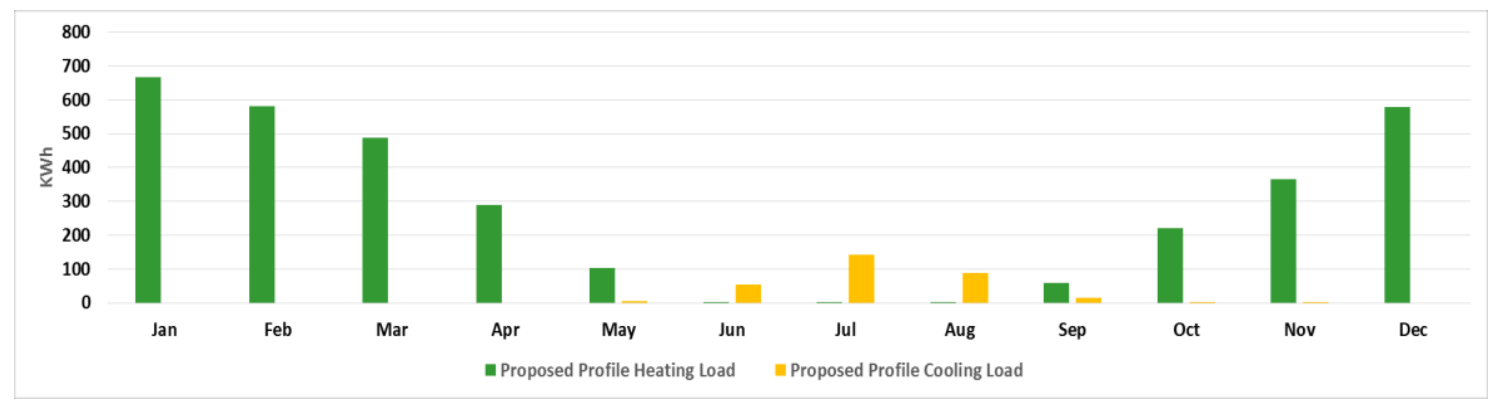

Figure 4.15 Annual heating and cooling loads based on proposed heating and cooling profile

Furthermore, to compare the heating loads between the proposed heating profile and a fixed profile (baseline), one of the ASHRAE standard profiles, ASHRAE 6AM-10PM-no lunch, is chosen which is compatible with this office occupants' schedule. The annual heating energy consumption has been calculated based on this ASHRAE standard profile as shown in Figure 4.16.

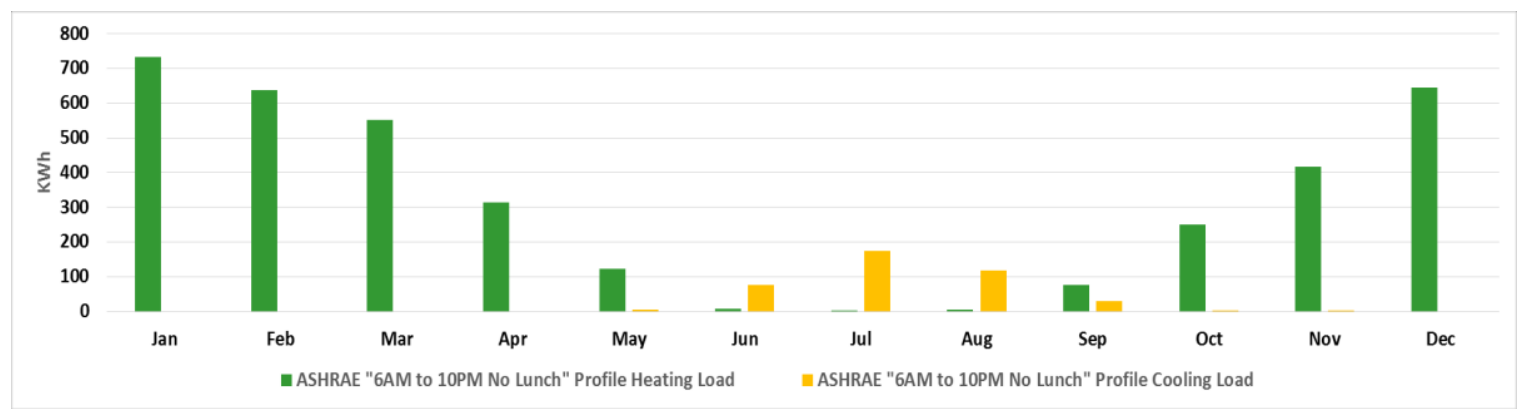

Figure 4.16 Annual heating and cooling loads based on ASHRAE 6AM-10PM-no lunch

Figure 4.17 compares the energy consumptions resulting from the implementation of proposed heating and cooling profile and ASHRAE standard profile. 


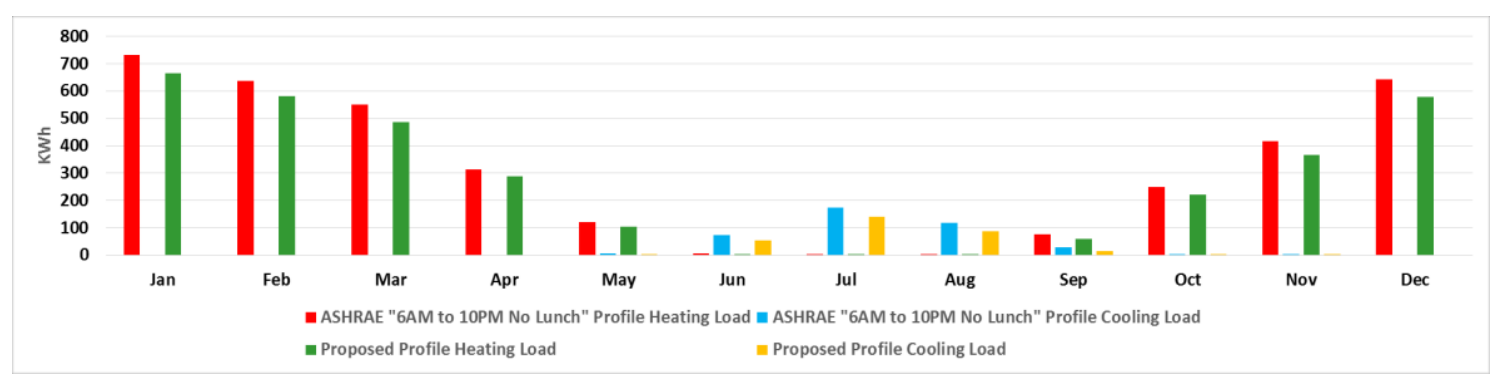

Figure 4.17 Comparison between the energy consumptions based on proposed heating and cooling profile and ASHRAE standard profile

\subsubsection{Lighting Profile}

As explained in Section 3.5.2, to simulate the lighting energy consumption, it is assumed that individual lighting fixture is assigned to each of the four occupants' zones (working space). It is determined that if, for example, occupant $\mathrm{N}$ is in her zone, her zone's lighting is $\mathrm{ON}$ and, in order to save energy, when she leaves her zone the light turns OFF.

Similar to the heating profile, daily lighting profiles are defined as series of hourly values applying at different times of weekdays and weekend days based on the overall occupancy profile. Figure 4.18 shows the lighting profiles created for each day of the week in IES $<$ VE $>$ based on monitored data. For instance, the number of occupants in the office between 9:00 and 10:00 AM was one on Monday, it means that $25 \%$ of the maximum value of the lighting was consuming. Therefore the value of $25 \%$ is assigned to that period. While there were three occupants in the office between 4:00 PM and 5:00 PM on the same day. Hence, 75\% of the maximum value of the lighting was consumed; and consequently, the value of $75 \%$ is assigned to that period. 


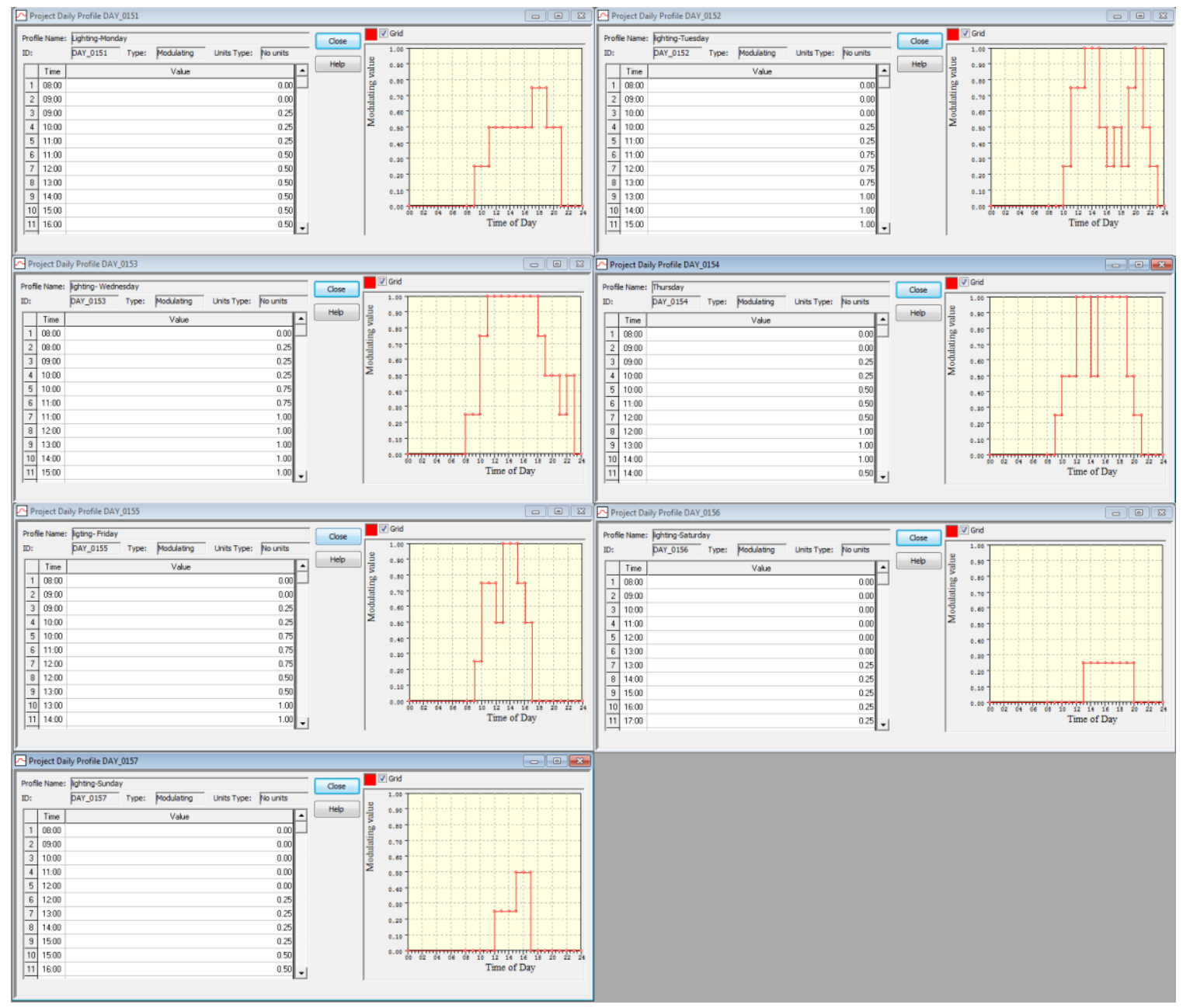

Figure 4.18 Weekly proposed lighting profile based on monitored data

To calculate the amount of lighting energy consumption, the maximum power consumption of the lighting fixtures should be assigned to the lighting profile. There are four lighting fixtures in the office, each of which includes two 32 Watt - T8 tubes. Therefore, the maximum power consumption of the lighting fixtures would be $256 \mathrm{~W}(4 *(2 * 32)=256 \mathrm{~W})$ which is assigned to the energy model's lighting profile settings. This proposed lighting weekly profile is assigned to the lighting section of the office model in the software to run the annual lighting energy consumption simulation. However, more details are required to get more accurate results. 
Furthermore, ASHRAE 6AM-10PM-no lunch profile is chosen to compare the lighting energy consumption based on the proposed lighting profile and a fixed profile. The comparison between the results from the proposed lighting profile and ASHRAE standard profile shows the difference in the amount of the energy consumption as shown in Figure 4.19.

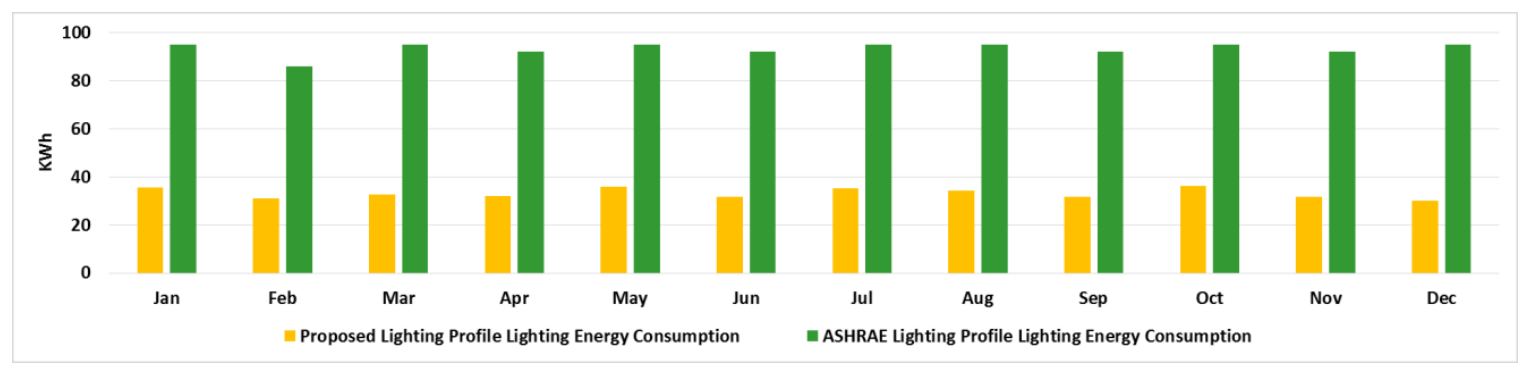

Figure 4.19 Comparison between the energy consumption based on proposed lighting profile and ASHRAE standard profile

\subsubsection{IT Equipment Profile}

As described in Sections 3.4.3 and 4.7, probabilistic individual occupancy profiles demonstrate the probability of the presence of each of the occupants during weekdays and weekend days on an hourly basis in the office. These probabilistic individual occupancy profiles are used to create IT equipment profiles for the energy consumption simulation. In the IT equipment energy simulation, it is assumed that if, for example, occupant $\mathrm{N}$ is in the office, her IT equipment is in use and consumes energy.

Moreover, the monitored data demonstrates that occupant $\mathrm{N}$ spent most of her times in her zone while she was in the office. Hence, to simplify the IT equipment profile, it is assumed that occupants are in their zones and use their IT equipment when they are in the office.

Similar to the heating and lighting profiles, the daily occupants' IT equipment profiles are defined as series of values applying at different times of weekdays and weekend days on an hourly basis based on probabilistic individual occupancy profiles. For instance, the probabilistic 
individual occupancy profiles in Figure 4.11 demonstrate that there is the possibility of $20 \%$ that occupant $\mathrm{S}$ be in the office between 9:00 AM and 10:00 AM during weekdays. Therefore there is the possibility of $20 \%$ of using IT equipment. Thus the value of $20 \%$ is assigned to the duration between 9:00 $\mathrm{AM}$ and 10:00 $\mathrm{AM}$ of occupant $\mathrm{S}$ IT equipment profile during weekdays. Moreover there is the possibility of around 5\% that the occupant $\mathrm{S}$ be in the office between 4:00 $\mathrm{PM}$ and 5:00 PM during weekend days, it means that there is the possibility of around 5\% of using IT equipment. Consequently the value of $5 \%$ is assigned to the duration between 4:00 PM and 5:00 PM of occupant S's IT equipment weekend days profile.

Similar to the heating, cooling, and lighting energy consumption profile, only annual and weekly profiles can be assigned to the energy model in the IES $<\mathrm{VE}>$. In this regard, first, daily profiles (i.e., weekdays and weekend days) are created. Then, these daily profiles are assigned to each day of the week based on probabilistic individual occupancy profile to generate weekly profiles. This individual proposed IT equipment profile is assigned to the energy model in the software to run the annual individual IT equipment energy consumption simulation.

To calculate the IT equipment energy consumption, its maximum power consumption should be assigned to the profile. Since occupancy behavior and their interactions with their IT equipment differ from one occupant to another, individual profiles are created for each of the occupants and are assigned to the model to calculate the amount of each of the occupants' energy consumption and the potential energy saving as explained in the following.

\subsubsection{Occupant L's Profile}

Occupant L's computer and monitor maximum power consumptions based on the average data from monitoring are $0.114 \mathrm{~kW}$ and $0.043 \mathrm{~kW}$, respectively. Therefore, the total power 
consumption would be $0.157 \mathrm{~kW}$. Figure 4.20 shows the results of the proposed weekly energy consumption profile which is simulated in the software.

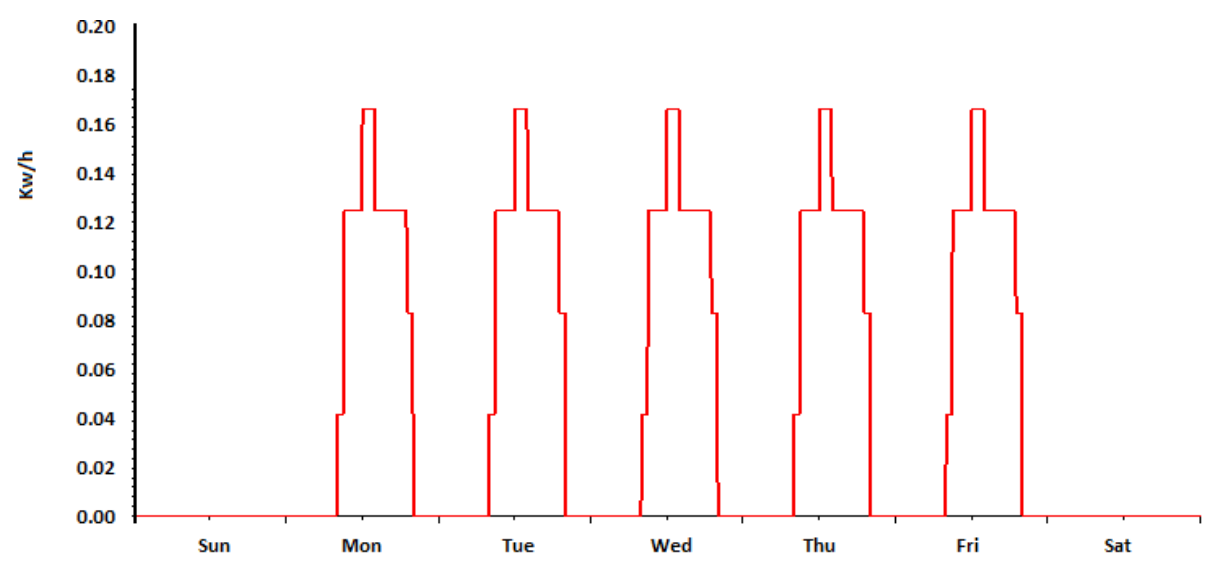

Figure 4.20 Occupant $L$ weekly IT equipment energy consumption profile

Furthermore, to compare the occupant L's IT equipment energy consumption based on proposed individual weekly profile and a fixed profile, ON continuous (always ON) profile, which is more compatible with this office occupancy behavior, is selected to run the simulation. This profile is chosen and assigned to the energy model in the software and annual individual IT equipment energy consumption is calculated. The comparison between the results from the proposed occupant L's IT equipment profile and the ON continuous (always $\mathrm{ON}$ ) profile shows the difference in the amount of the energy consumption and energy saving which are shown in Figure 4.21.

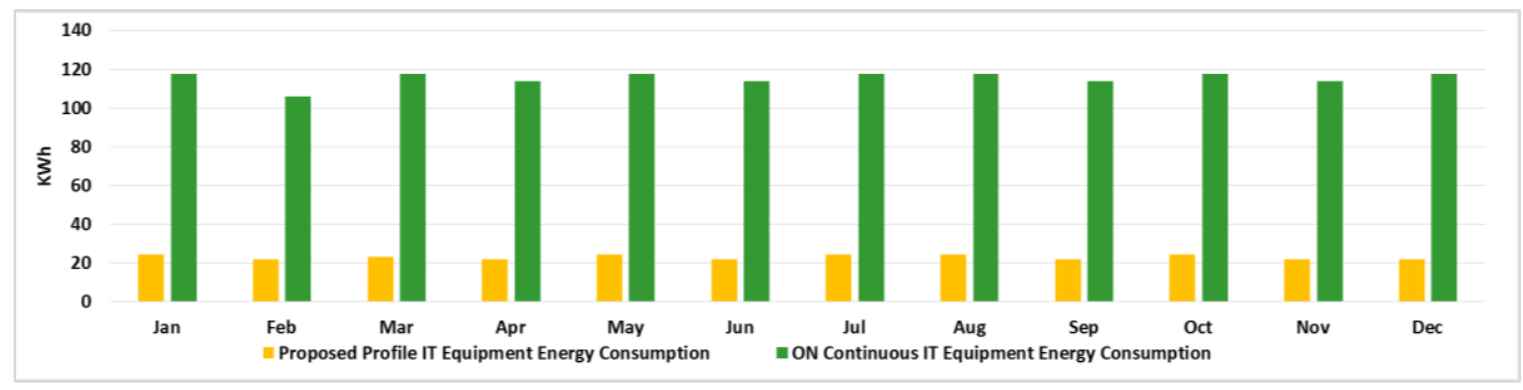

Figure 4.21 Comparison between the energy consumption based on proposed occupant L IT equipment profile and $O N$ continuous profile 


\subsubsection{Occupant M's Profile}

Occupant M's computer and monitor maximum power consumptions are $0.065 \mathrm{~kW}$ and 0.043 $\mathrm{kW}$, respectively. Therefore, the total power consumption would be $0.108 \mathrm{~kW}$.

Figure 4.22 shows the results of the proposed weekly energy consumption profile.

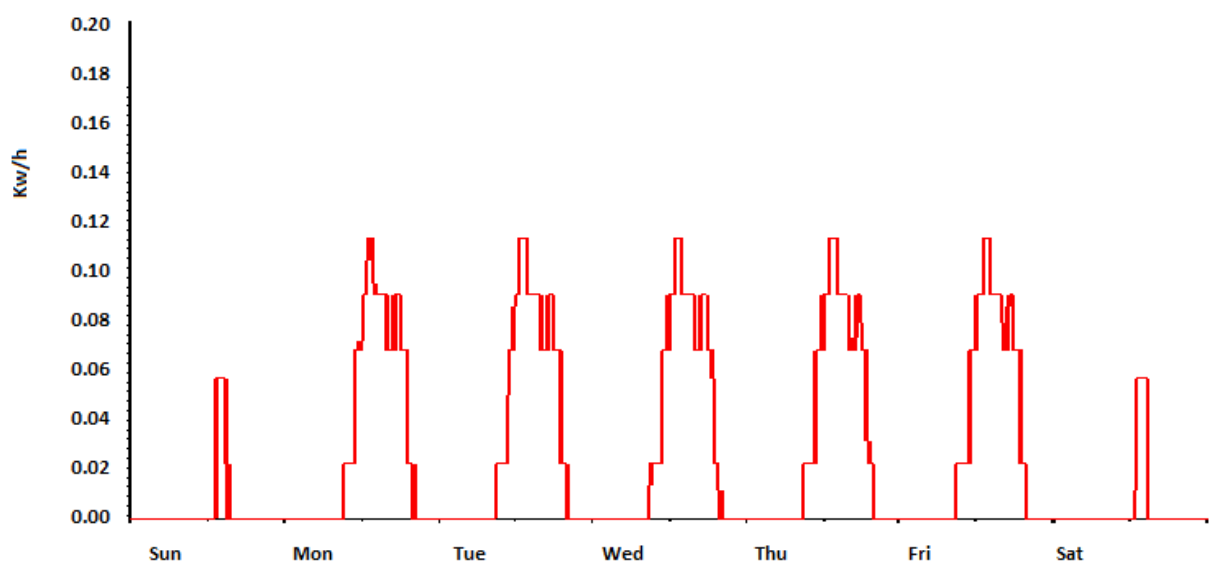

Figure 4.22 Occupant M weekly IT equipment energy consumption profile

The comparison between the proposed occupant M's IT equipment profile and ON continuous (always ON) profile shows the difference in the amount of the energy consumption as shown in

Figure 4.23.

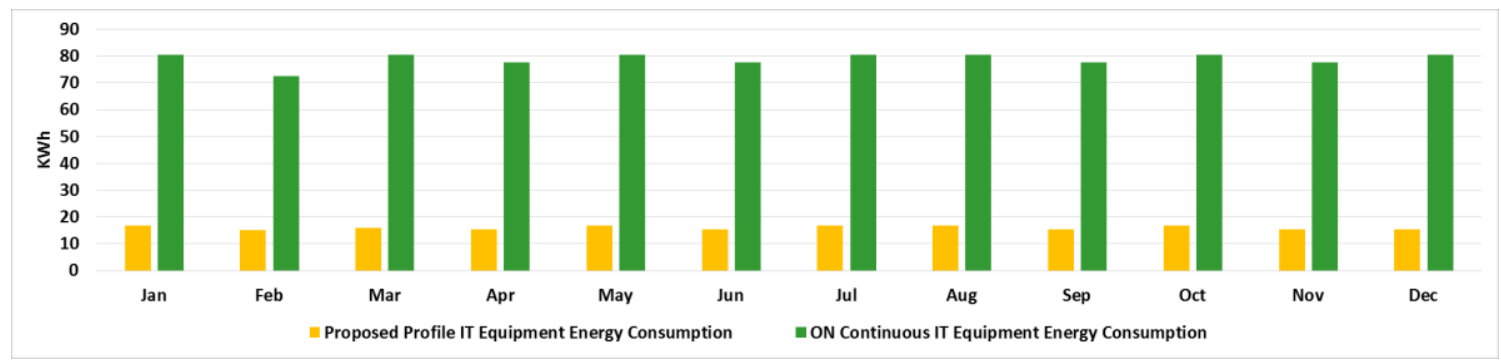

Figure 4.23 Comparison between the energy consumption based on proposed occupant M IT equipment profile and $O N$ continuous profile 


\subsubsection{Occupant N's Profile}

Occupant N's computer and monitor maximum power consumptions are $0.034 \mathrm{~kW}$ and 0.024 $\mathrm{kW}$, respectively. Therefore, the total power consumption would be $0.058 \mathrm{~kW}$. Figure 4.24 shows the results of the proposed weekly energy consumption profile.

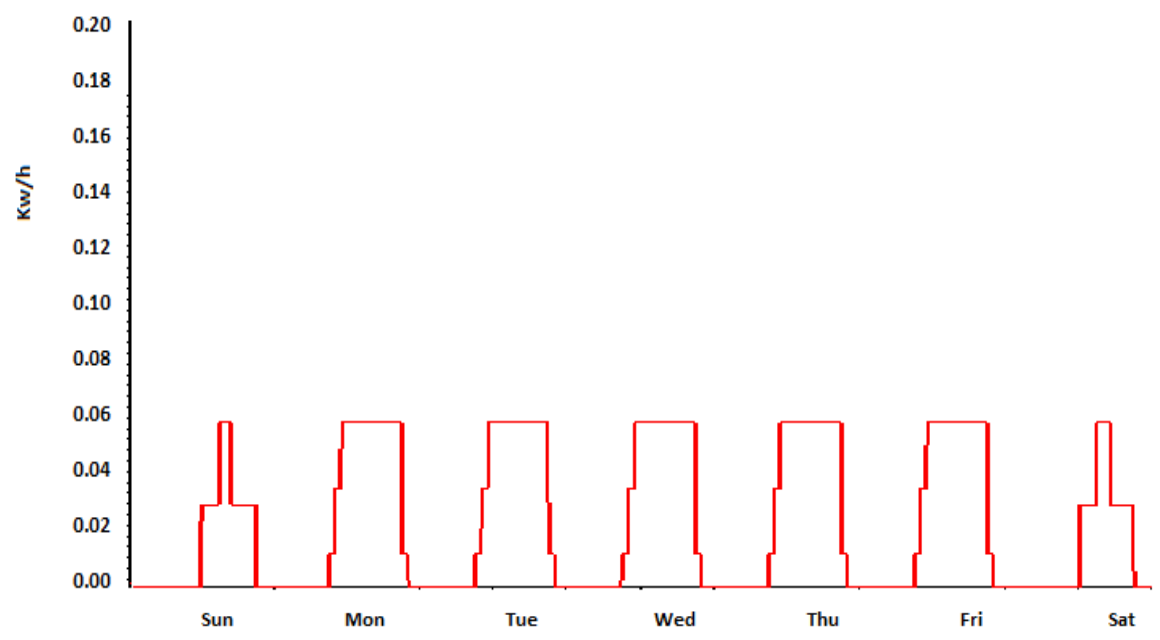

Figure 4.24 Occupant $\mathrm{N}$ weekly IT equipment energy consumption profile

The comparison between the results of the proposed occupant N's IT equipment profile and ON continuous (always $\mathrm{ON}$ ) profile shows the difference in the amount of the energy consumption as shown in Figure 4.25.

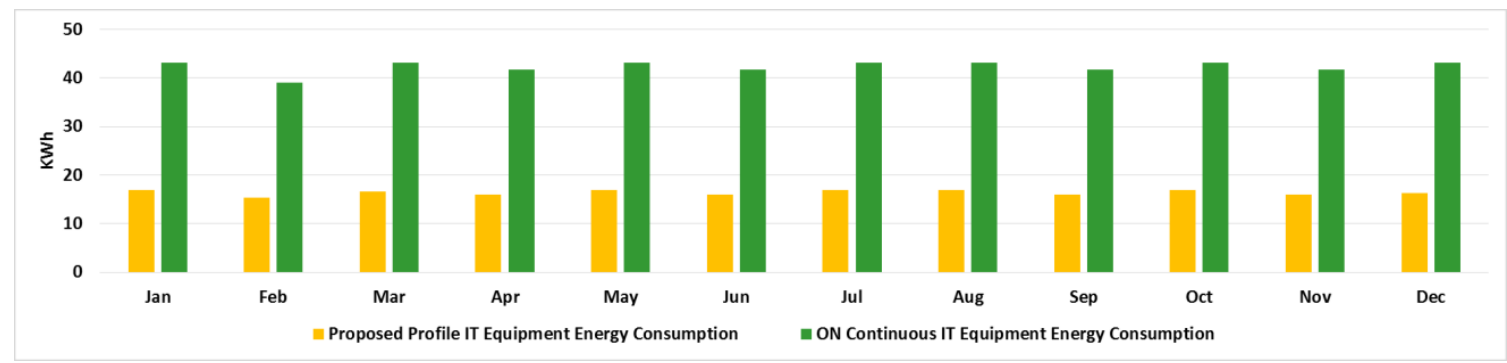

Figure 4.25 Comparison between the energy consumption based on proposed occupant N IT equipment profile and $O N$ continuous profile 


\subsubsection{Occupant S's Profile}

Occupant S's computer and monitor maximum power consumptions are $0.087 \mathrm{~kW}$ and 0.090 $\mathrm{kW}$, respectively. Therefore, the total power consumption would be $0.177 \mathrm{~kW}$. Figure 4.26 shows the results of the proposed weekly energy consumption profile.

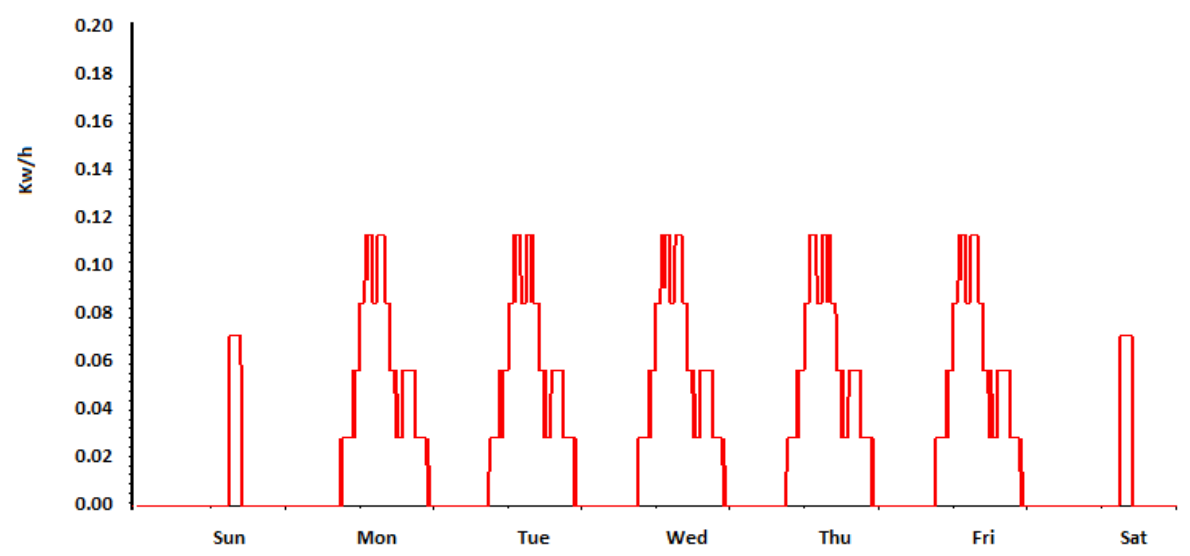

Figure 4.26 Occupant S weekly IT equipment energy consumption profile

The comparison between the results of the proposed occupant S's IT equipment profile and ON continuous (always $\mathrm{ON}$ ) profile shows the difference in the amount of the energy consumption as shown in Figure 4.27.

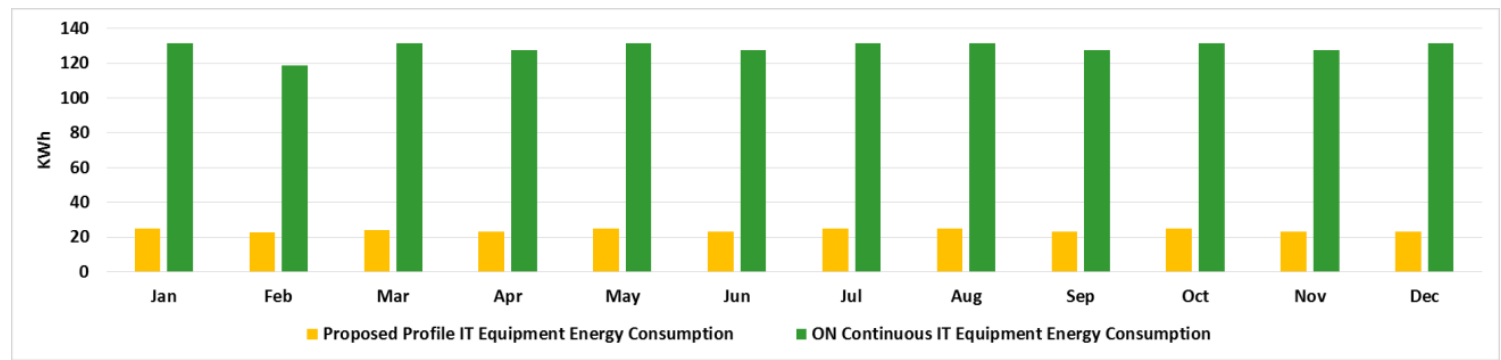

Figure 4.27 Comparison between the energy consumption based on proposed occupant S IT equipment profile and $\mathrm{ON}$ continuous profile 


\subsection{Energy Consumption Comparison between Proposed Profiles and Fixed Profiles}

In conclusion, the comparison between the results shows approximately $11 \%$ and $24 \%$ saving for heating loads and cooling loads, respectively. The proposed method profiles also resulted in up to $65 \%$ and $78 \%$ reduction for lighting and IT equipment energy consumption in the office, respectively. The results are summarized in Table 4.3 .

Table 4.3 Energy consumption comparison between proposed profiles and fixed profile

\begin{tabular}{|l|c|c|c|}
\hline & $\begin{array}{c}\text { Fixed profile } \\
\text { (KWh) }\end{array}$ & $\begin{array}{c}\text { Proposed method } \\
\text { profiles (KWh) }\end{array}$ & $\begin{array}{c}\text { Percentage of } \\
\text { saving (\%) }\end{array}$ \\
\hline Heating load & 3758.80 & 3356.20 & 10.71 \\
\hline Cooling load & 403.10 & 306.00 & 24.08 \\
\hline Lighting energy consumption & 1121.20 & 397.60 & 64.53 \\
\hline Equipment energy consumption & 4388.80 & 952.00 & 78.30 \\
\hline
\end{tabular}

\subsection{Summary and Conclusions}

A test was carried in one of the research offices at Concordia University to collect the required data to evaluate the impact of occupancy behavior on IT equipment energy consumption. The data was collected as a sample to create office occupancy profiles. UWB RTLS was used for occupancy location monitoring and ZigBee wireless energy meters, including a gateway and nine energy meters, were used for monitoring the IT equipment. The test was carried out for one week of data acquisition. An UWB sensor cell, consisting of four sensors and four tags, was used to $\log$ the data. Four occupants were asked to participate in this test and wear slim tags during the test period with an individual update rate of two readings per minute. Simultaneously, energy meters were attached to the occupants' IT equipment (i.e., four monitors, four computers, and a printer) with an individual update rate of two readings per minute in average to collect the energy consumption information. 
The Ubisense and Smartenit loggers were installed on a machine for logging the UWB RTLS and the ZigBee energy meters data during the test. A worksheet was developed in Excel to analyze the collected data. The occupancy maps were created to show occupants' locations in the office based on UWB coordinates (X, Y) while occupancy charts were developed to illustrate occupants' daily presence. Daily energy consumption graphs were created to show the monitors, computers, and printer energy consumption.

The data gained from the UWB occupancy monitoring were used to create the overall occupancy profiles and the individual probabilistic occupancy profiles. Based on the overall occupancy profiles, daily profiles were defined as series of values applying at different times of each day of the week on an hourly basis which were used to generate the heating, cooling, and lighting profiles. On the other hand, the probabilistic individual occupancy profiles were created for each of the occupants for the weekdays and weekend days, separately. The probabilistic individual occupancy profiles were used to generate individual occupant IT equipment profiles.

Results from the test demonstrate that there are two different types of behavior regarding saving energy in the office: (1) turning off IT equipment when not in use (user not in zone or out of the office even for a short time), which results in reducing the energy consumption; and (2) leaving IT equipment on all days long. In conclusion, as an example, it was estimated that if occupant L's monitor and computer were off during the time he was not in the office, about $0.38 \mathrm{KWh}$ $(20 \%)$ and $6.58 \mathrm{KWh}(83 \%)$ of energy could have been saved, respectively.

The 3D model was created in Revit. The gbXML format of the file was imported into the IES $<$ VE $>$ software to run the simulation. Annual and weekly profiles are the only two types of the profile which could be assigned to the model in the software. In this regard, daily profiles 
were created individually to be assigned to each day of the week to generate weekly profiles. The proposed weekly profiles were assigned to the office model in the software to run the annual energy consumption simulation.

Furthermore, a fixed profile was chosen as a base line to compare the energy consumption based on the proposed profiles and fixed profiles. The results demonstrates that applying a fixed schedule to run IT equipment in an office will result in a significant waste of energy during long periods of time especially during weekend days, holidays, and summer breaks. Therefore, dynamic occupancy-driven profiles would reduce the energy consumption. 


\section{CHAPTER 5 Conclusions and Future works}

\subsection{Summary of Research}

This thesis reviewed the concepts, techniques, standards, and main technologies that are used in the current research. The literature review included the information about RTLSs technologies, RFID based localization techniques and smart metering. RTLSs have been applied in previous studies for identifying, monitoring and localizing purposes, whereas smart metering is a common technique used to measure the energy consumption of equipment.

However, occupant activities and behaviors have a large impact on energy consumed in different buildings' sectors (i.e. residential, offices and retail sectors). Therefore, to evaluate the impact of the occupancy behavior on energy consumption, there are some limitations in these studies regarding the accuracy and the lack of statistical data. A significant amount of energy spent in these buildings could be saved by regulating installations and IT equipment according to actual needs. In order to realize this approach, occupant activities and behaviors are required as the most important input for generating occupant simulation profiles.

The proposed method in this research evaluates the occupancy behaviors and their impact on energy saving potential. A new sensor network method for real-time occupants and energy consumption monitoring was proposed. The sensor networks provided basic tools for gathering the information on user behavior and its interaction with IT equipment. UWB technology was selected in this research as an RTLS tool due to its high accuracy and ability to carry signals through doors and other obstacles and ZigBee products were chosen as energy meters due to its 
low-cost and low-power wireless sensor. The basics of UWB technology and ZigBee energy meters, including components and details about their operation were discussed.

Assessing the feasibility of this framework in real-world situation included monitoring occupancy location and presence as well as measuring IT equipment energy consumption in order to evaluate the impact of occupancy behavior on energy saving. Occupancy profiles are created through sensing and data processing. Occupancy profiles can enable accurate building energy management based on monitoring an office environment resulting in annual energy saving in HVAC, lighting, and IT equipment.

\subsection{Research Contributions and Conclusions}

A test was carried in one of the research offices at Concordia University for one week to collect the required data to evaluate the impact of occupancy behavior on IT equipment energy consumption. The data was collected as a sample to create office occupancy profiles. UWB RTLS was used for occupancy location monitoring and ZigBee wireless energy meters were used for monitoring the IT equipment. An UWB sensor cell, consisting of four sensors and four tags, was used to log the data. Four occupants were asked to participate in this test and wear slim tags during the test period with an individual update rate of two readings per minute. Simultaneously, energy meters were attached to the occupants' IT equipment (i.e., four monitors, four computers, and a printer) with an individual update rate of two readings per minute in average to collect the energy consumption information.

The Ubisense and Smartenit loggers were installed on a machine for logging the UWB RTLS and the ZigBee energy meters data during the test. A worksheet was developed in Excel to analyze the collected data. The occupancy maps were created to show occupants' locations in the 
office based on UWB coordinates (X, Y) while occupancy charts were developed to illustrate occupants' daily presence. Daily energy consumption graphs were created to show the monitors, computers, and printer energy consumption.

The data gained from the UWB occupancy monitoring were used to create the overall occupancy profiles and the individual probabilistic occupancy profiles. Based on the overall occupancy profiles, daily profiles were defined as series of values applying at different times of each day of the week on an hourly basis which were used to generate the heating, cooling, and lighting profiles. On the other hand, the probabilistic individual occupancy profiles were created for each of the occupants for the weekdays and weekend days, separately. The probabilistic individual occupancy profiles were used to generate individual occupant IT equipment profiles.

Results from the test demonstrate that there are two different types of behavior regarding saving energy in the office: (1) turning off IT equipment when not in use (user not in zone or out of the office even for a short time), which results in reducing the energy consumption; and (2) leaving IT equipment on all days long. In conclusion, as an example, it was estimated that if occupant L's monitor and computer were off during the time he was not in the office, about $0.38 \mathrm{KWh}$ $(20 \%)$ and $6.58 \mathrm{KWh}(83 \%)$ of energy could have been saved, respectively.

The 3D model was created in Revit. The gbXML format of the file was imported into the IES $<\mathrm{VE}>$ software to run the simulation. Annual and weekly profiles are the only two types of the profile which could be assigned to the model in the software. In this regard, daily profiles were created individually to be assigned to each day of the week to generate weekly profiles. The proposed weekly profiles were assigned to the office model in the software to run the annual energy consumption simulation. 
Furthermore, a fixed profile was chosen as a base line to compare the energy consumption based on the proposed profiles and fixed profiles. The comparison between the results shows approximately $11 \%$ and $24 \%$ saving for heating loads and cooling loads, respectively. The proposed method profiles also resulted in up to $65 \%$ and $78 \%$ reduction for lighting and IT equipment energy consumption in the office, respectively. The results demonstrate that applying a fixed schedule to run IT equipment in an office will result in a significant waste of energy during long periods of time especially during weekend days, holidays, and summer breaks. Therefore, dynamic occupancy-driven profiles would reduce the energy consumption.

\subsection{Limitations and Future Work}

Time limitation prevented sufficient periods of data acquisition in this thesis. The number and the composition of the office occupants can be varied regarding the seasons of the year. Therefore, more data is required to be collected to create more accurate occupancy profiles.

Occupancy profiles can make timely adjustments to the systems possible. In the future work, the occupancy profiles can be used to proactively and reactively control and optimize the building energy consumption while responding to comfort preferences of the occupants to support the implementation of demand-driven HVAC, lighting, and IT equipment operations. The controlling part improves the performance of the building in two aspects, saving energy (e.g. turning off the lights after the occupants leave the office) and occupants' satisfaction (e.g. turning on the HVAC prior to occupants' arrival). Besides, another type of profile can be created based on monitored equipment energy consumption which can be used to calibrate the occupancy profiles. UWB RTLS is a method to collect real-time occupancy information and to create occupancy profiles. Although implementing UWB in small indoor environments can be 
expensive, the cost can be reduced if it is used for several purposes such as security, facility management, and emergency situations.

Moreover, a questionnaire can be prepared to collect the information about occupants' preferred temperature and humidity levels. The collected data from occupants can be used to define occupants' comfort level. Temperature and humidity sensors can be used to monitor the office environment temperature and relative humidity in the future work. By comparing the collected data from these sensors with the occupants' preferences, operators can easily decide that if any adjustment is required to be undertaken.

Furthermore, it is important to engage building occupants in the building energy management systems since occupancy behavior can have a significant impact on building energy consumption. A feedback system can provide historical and current equipment energy consumption measured by smart meters. In order to involve the occupants, these information can be sent to them on a regular basis to inform them about their energy consumption which can increase occupants' awareness about their behavior. 


\section{REFERENCES}

Aggarwal, J.K., and M.S. Ryoo. 2011. "Human Activity Analysis: A Review." ACM Computing Surveys, 43 (3).

Allcott, H. 2010. "Behavior and Energy Policy." Science, 327(5970): 1204-1205.

ASHRAE. 2002. ASHRAE Guideline 14 for Measurement of Energy and Demand Savings. American Society of Heating, Refrigeration and Air Conditioning Engineers, Atlanta, GA.

Attia, A., L. Beltrán, A.D. Herde, and J. Hensen. 2009. "Architect Friendly: A Comparison of Ten Different Building Performance Simulation Tools." Eleventh International IBPSA Conference, Glasgow, Scotland.

Bourgeois, D. 2005. "Detailed Occupancy Prediction, Occupancy-Sensing Control and Advanced Behavioural Modelling within Whole-Building Energy Simulation." l'Université Laval, Canada.

Conti, J., P. Holtberg, J. A. Beamon, S. Napolitano, A. M. Schaal, J. T. Turnure, and L. Westfall. 2013. The International Energy Outlook. U.S. Energy Information Administration (EIA). Washington.

Croome, D.C. 2011. "Sustainable Intelligent Buildings for People: A review." Intelligent Buildings International, 3(2): 67-86.

Delaney, D.T., G.M.P. O’Hare, and A.G. Ruzzelli. 2009. "Evaluation of Energy-Efficiency in Lighting Systems Using Sensor Networks." Proceedings of the First ACM Workshop on 
Embedded Sensing Systems for Energy-Efficiency in Buildings, BuildSys '09. New York: ACM. $61-66$.

Ergen, S.C. 2004. "ZigBee/IEEE 802.15. 4 Summary." UC Berkeley 10.

Erickson, V., M. Carreira-Perpinan, and A. Cerpa. 2010. "OBSERVE: Occupancy-Based System for Efficient Reduction of HVAC Energy for Efficient Reduction of HVAC Energy." 2nd ACM Workshop on Embedded Sensing Systems for Energy-Efficiency in Building, BuildSys '10. New York: ACM.

Erickson, V.L., Y. Lin, A. Kamthe, B. Rohini, A. Surana, A.E. Cerpa, M.D. Sohn, and S. Narayanan. 2009. "Energy Efficient Building Environment Control Strategies Using Realtime Occupancy Measurements." 1st ACM Workshop on Embedded Sensing Systems for EnergyEfficiency in Buildings. BuildSys '09. ACM. 19-24.

Faruqui, A., S. Sergici, and A. Sharif. 2010. "The Impact of Informational Feedback on Energy Consumption—A Survey of the Experimental Evidence." Energy, 35(4): 1598-1608.

Fischer, C. 2008. "Feedback on Household Electricity Consumption: A Tool for Saving Energy?" Energy Efficiency, 1(1): 79-104.

Gillott, M., R. Holland, S. Riffat, and J.A. Fitchett. 2006. "Post-Occupancy Evaluation of Space Use in a Dwelling Using RFID Tracking." Architectural Engineering and Design Management, 2(4): $273-288$.

Goldstein, R., A. Tessier, and A. Khan. 2011. "Space Layout in Occupant Behavior Simulation." International IBPSA Conference, Sydney, Australia. 
Hagras, H., V. Callaghan, M. Colley, G. Clarke, A. Pounds-Cornish, and H. Duman. 2004. "Creating an Ambient-Intelligence Environment Using Embedded Agents, Intelligent Systems." IEEE. 12-20.

Harle, R. K., and A. Hopper. 2008. "The Potential for Location-Aware Power Management." 10th international conference on Ubiquitous computing. New York: ACM. 302-311.

Harris, C., and V. Cahill. 2005. "Exploiting User Behaviour for Context-Aware Power Management." Wireless and Mobile Computing, Networking and Communications. Montreal: IEEE. 122-130.

Hoes, P., J.L.M. Hensen, M.G.L.C. Loomans, B. De Vies, and D. Bourgeois. 2009. "User Behavior in Whole Building Simulation." Energy and Buildings, 41(3), 8 pages. 41(3): 295-302.

Hong, T, H.W. Lin, W.K. Chang, L. Wang, W. Turner, D. Yan, D. Zhu, et al. 2013. Building Performance Simulation. Berkeley: Ernest Orlando Lawrence Berkeley National Laboratory.

Jazizadeha, F., G. Kavulya, J.Y. Kwak, B. Becerik-Gerber, M. Tambe, and W. Wood. 2012. "Human-Building Interaction for Energy Conservation in OfficeBuildings." Construction Research Congress.

Kamthe, A., L. Jiang, M. Dudys, and A. Cerpa. 2009. "SCOPES: Smart Cameras Object Position." 6th European Conference on Wireless Sensor Networks (EWSN), EWSN '09. Springer-Verlag, Berlin, Heidelberg: EWSN. 279-295.

Kim, Y., Z. Charbiwala, A. Singhania, T. Schmid, and M. B. Srivastava. 2008. "SPORTLIGHT: Personal Natural Resource Consumption Profiler." Charlottesville: HotEmNets. 
Krygiel, E., and B. Nies. 2008. Green BIM:Successful Sustainable Design with Building Information Modeling. Indianapolis: Wiley.

Lee, S.W., and K. Mase. 2002. "Activity and Location Recognition Using Wearable Sensors." Pervasive Computing 1 (3): 24-32.

Levine, M., D. Ürge Vorsatz, K. Blok, L. Geng, D. Harvey, S. Lang, G. Levermore, et al. 2007. Residential and Commercial Buildings. Cambridge and New York: Cambridge University Press.

Li, N., G. Calis, and Burcin B.G. 2012. "Measuring and Monitoring Cccupancy with an RFID Based System for Demand-Driven HVAC Operations." Automation in Construction, 24: 89-99.

Li, Nan, and B. Becerik-Gerber. 2011. "Performance-Based Evaluation of RFID-Based Indoor Location Sensing Solutions for the Built Environment." Advanced Engineering Informatics, $535-546$.

Liu, H., H. Darabi, P. Banerjee, and J. Liu. 2007. "Survey of Wireless Indoor Positioning Techniques and Systems." IEEE Transactions on: Systems, Man, and Cybernetics, Part C: Applications and Reviews, 37(6): 1067-1080.

Malik, A. 2009. RTLS for Dummies. Indianapolis, Indiana: Wiley. Accessed November 8, 2013. <http://www.wiley.com/WileyCDA/WileyTitle/productCd-047039868X.html>.

Marchiori, A., and Q. Han. 2010. "Distributed Wireless Control for Building Energy Management." Proceedings of the 2nd ACM Workshop on Embedded Sensing Systems for Energy-Efficiency in Building, BuildSys '10. New York: ACM. 37-42. 
Masoudifar, N., A. Hammad, and M Rezaee. 2014. "Monitoring Occupancy and Office Equipment Energy Consumption Using Real-Time Location System and Wireless Energy Meters." Winter Simulation Conference. Savannah, GA.

Menezes, A.C., A. Cripps, R.A Buswell, and D. Bouchlaghem. 2013. "Benchmarking Small Power Energy Consumption in Office Buildings in the United Kingdom: A Review of Data Published in CIBSE Guide F." Building Services Engineering Research and Technology, 34: 7386.

Munoz, D., F. B. Lara, C. Vargas, and R. Enriquez-Caldera. 2009. "Position Location Techniques and Applications." Academic Press: 1st Edition. Mexico.

National Resource Canada. 2011. 15th Edition of Energy Efficiency Trends in Canada. Ottawa: National Resource Canada. Accessed October 28, 2013.<www.nrcan.gc.ca.>

Newsham, G.R., and B.J. Birt. 2010. "Building-Level Occupancy Data to Improve ARIMABased Electricity Use Forecasts." 2nd ACM Workshop on Embedded Sensing Systems for Energy-Efficiency in Building, BuildSys '10. New York: ACM. 13-18.

Nguyen, T.A., and M. Aiello. 2013. "Energy intelligent buildings based on user activity: A Survey." Energy and Buildings, 56:244-257.

Padmanabh, K., A. Malikarjuna, V.S. Sen, S.P. Katru, A. Kumar, S.P.C.S.K. Vuppala, and S. Paul. 2009. "iSense: A Wireless Sensor Network Based Conference Room Management System." First ACM Workshop on Embedded Sensing Systems for Energy-Efficiency in Buildings. New York: ACM. 37-42. 
Page, J. 2007. "Simulating Occupant Presence and Behaviour in Buildings." PhD Thesis, École Polytechnique Fédérale de Lausanne. Switzerland.

Page, J., D. Robinson, N. Morel, and J.L. Scartezzini. 2008. "A Generalised Stochastic Model for the Simulation of Occupant Presence." Energy and Buildings, 40(2): 83-98.

Peschiera, G., and J. E Taylor. 2012. "The Impact of Peer Network Position on Electricity Consumption in Building Occupant Networks Utilizing Energy Feedback Systems." Energy and Buildings, 49:584-590.

Petersen, J. E., V. Shunturov, K. Janda, G. Platt, and K. Weinberger. 2007. "Dormitory Residents Reduce Electricity Consumption When Exposed to Real-Time Visual Feedback and Incentives." International Journal of Sustainability in Higher Education, 8(1):16-33.

Rodriguez, S. 2010. "Experimental Study on Location Tracking of Construction Resources Using UWB for Better Productivity and Safety." MA.Sc. Thesis, Concordia University. Montreal, Canada

Sana. 2013. "A Survey of Indoor Localization Techniques." IOSR Journal of Electrical and Electronics Engineering, 6(3): 69-76.

Shalini, S.M. 2011. "Evaluating the Ability of eQUEST Software to Simulate Low-energy Buildings in a Cold Climatic Region." MA.Sc. Thesis, University of Toronto, Toronto, Canada.

Singh, V. 2011. "Analysis Methods for Post Occupancy Evaluation of Energy-Use in High Performance Buildings Using Short-Term Monitoring." MA.Sc. Thesis, Arizona State University. Phoenix, Arizona. 
Sohraby, K., D. Minoli, and T. Znati. 2007. "Introduction and Overview of Wireless Sensor Networks." In Wireless Sensor Network Technology, Protocols, and Applications, 1-30. John Wiley and Sons, Inc., Hoboken, New Jersey.

Smartenit. 2013. Accessed November 7, 2013. <http://simplehomenet.com.>

Spataru, C., and M. Gillott. 2012. "Using a RTL System Based on RFID Technology for Monitoring Occupants Domestic Energy Use and Behaviour." 3rd International Conference on Sustainability of Energy and Buildings. Smart Innovation, Systems and Technologies. 471-480.

Spataru, C., M. Gillott, and M.R. Hall. 2010. "Domestic Energy and Occupancy: A Novel PostOccupancy Evaluation Study." Low-Carbon Technologies, 5(3): 148-157.

Sun, Z., Wang, S., Ma, Z. 2011. "In-Situ Implementation and Validation of a CO2 Based Adaptive Demand-Controlled Ventilation Strategy in a Multi-Zone Office Building." Building Environment, 46(1):124-133.

Tabak, V. 2008. "User Simulation of Space Utilisation: System for Office Building Usage Simulation." PhD Thesis, Technische Universiteit Eindhoven. Eindhoven, Netherlands:

Tachwali, Y., Refai, H., Fagan, J. E. 2007. "Minimizing HVAC Energy Consumption Using a Wireless Sensor Network." 33rd Annual Conference of the IEEE Industrial Electronics. IEEE. $439-44$.

Ubisense. 2013. Accessed November, 2013. <http://www.ubisense.ne.> 
Ueno, T., F. Sano, O. Saeki, and K. Tsuji. 2006. "Effectiveness of an Energy-Consumption Information System on Energy Savings in Residential Houses Based on Monitored Data." Applied Energy, 83:166-183.

Vassileva, I., M. Odlare, F. Wallin, and E. Dahlquist. 2012. "The Impact of Consumers' Feedback Preferences on Domestic Electricity Consumption." Applied Energy, 39:575-582.

Ward, A. M. R. 2007. "In-Building Location Systems". Proceedings of the the Institution of Engineering and Technology Seminar on Location Technologies. London, U.K. 1-18.

Ward, A. M. R., and P. M. Webster. 2009. "Location Device and System using UWB and NonUWB Signals". U.S Patent US 7636062 B2. December 22.

Wilke, U., F. Haldi, J.L. JScartezzini, and D. Robinson. 2012. "A Bottom-Up Stochastic Model to Predict Building Occupants’ Time-Dependent." Building and Environment, 60:254-264.

Wong, J., H. Li, and S. Wang. 2005. "Intelligent Building Research: A Review." Automation in Construction, 14(1): 143-159.

Yang, G. 2012. "Energy Simulation in Existing Buildings: Calibrating the Model for Retrofit Studies." Report, Faculty of the USC School of Architecture, University of Southern California, California.

Yang, Z., N. Li, b. Becerik-Gerber, and M. Orosz. 2012. "A Non-Intrusive Occupancy Monitoring System for Demand Driven HVAC Operations." Construction Research Congress. West Lafayette: American Society of Civil Engineers (ASCE). 828-837. 
Yu, T. 2010. "Modeling Occupancy Behavior for Energy Efficiency and Occupants Comfort." ICMLA '10 Proceedings of the Ninth International Conference on Machine Learning and Applications. Washington: ICMLA 2010. 726-731.

ZigBee Alliance. 2013. Accessed November, 2013. <https://www.ZigBee.org>. 


\section{APPENDICES}

\section{Appendix A- Ubisense Series 7000 Slim Tag Personnel or Assets}

The Ubisense slim tag is a small device which is used as a tracking technology. Its features are LEDs for easy identification, a buzzer to provide basic messaging capabilities, a motion detector to instantly activate a stationary tag and two push buttons to trigger events which are shown in Figure A.1 and its specifications are explained in Table A.1. (Ubisense, 2013). When the slim tag is attached to assets or worn by people, it allows them to be positioned to an accuracy of $15 \mathrm{~cm}$ in $3 \mathrm{D}$ in real-time.
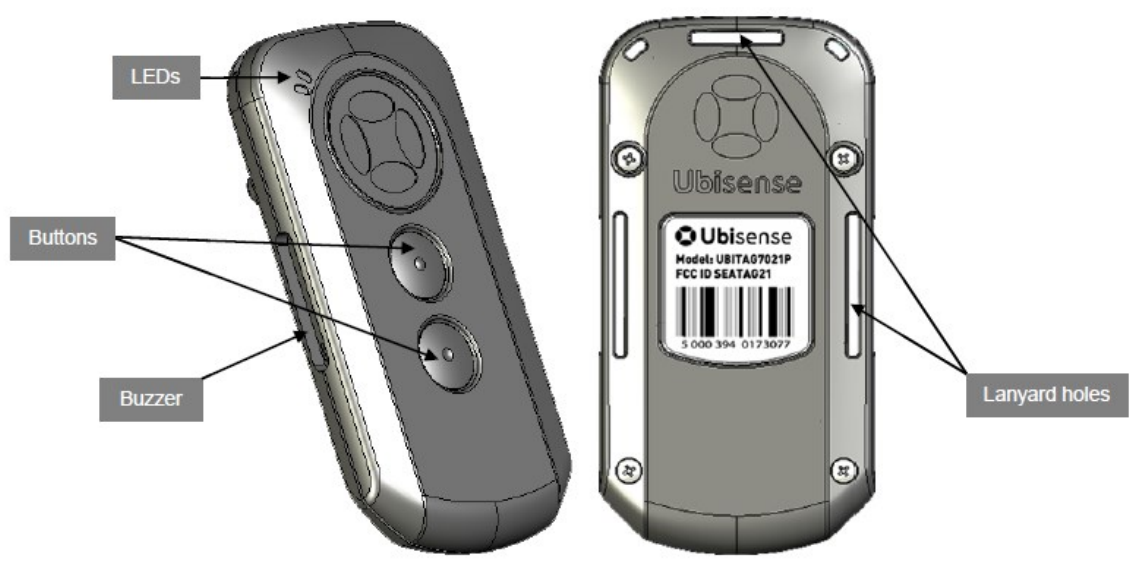

Figure A.1 UWB slim tag component (Ubisense, 2013)

\section{- Accurate Location}

Ubisense location system uses UWB radio pulses which are transmitted by slim tags, to find its position to within $15 \mathrm{~cm}$ in $3 \mathrm{D}$. Accurate data is maintained by UWB even in cluttered, multipath-rich indoor environments. The Ubisense UWB location system collects data be 
measuring both AoA and TDoA of the tag's signals to create accurate 3D tracking information even when there are only two sensors to detect the tag (Ubisense, 2013).

\section{- Two-way Communication}

UWB uses one-way UWB radio for tracking. In addition to the one-way radio, Ubisense tags employ unique dual-radio architecture and tags have a conventional bi-directional $2.4 \mathrm{GHz}$ radio for control and telemetry. The Ubisense system two-way communication manages the tag's update rate, flash the tag's LEDs or sound its buzzer, report battery status and button presses, and wirelessly reprogram the tag to enable new features dynamically (Ubisense, 2013).

\section{- Flexible Update Rates}

The Ubisense software platform sets the tag's update rate dynamically and automatically varied depending on tag activity. A high update rate is assigned to the quickly moving tags for best tracking and the update can be reduced for best battery lifetime if it is moving slowly. The tags sleep to conserve power when it is stationary. An inbuilt motion detector ensures the tag transmits again as soon as it is moved (Ubisense, 2013).

\section{- User Interaction Features}

The tag has two buttons which provides context-sensitive input for interactive systems. At the time a tag button is pressed, applications use the tag's location to determine what action should be taken in response such as activating an item of machinery when a button is pressed and the user is in a safe location. The applications are also used to send feedback through the tag LEDs or the buzzer to the user to demonstrate that the application has received the service request (Ubisense, 2013). 


\section{- Rugged and Adaptable}

The Slim Tag is designed to be dust-and water-resistant, mechanically robust, and securely mounted or worn using a variety of attachment mechanisms for use in a variety of environments (Ubisense, 2013).

\section{- Long Battery Life}

The tag's long battery lifetimes are due to its low current consumption and power management techniques. For example, the tag has an expected lifetime of over four years when it is used to locate an office worker every three seconds during working hours. Batteries can simply be replaced and status reports and alerts make maintenance easy (Ubisense, 2013).

Table A.1 Slim tag specifications (Ubisense, 2013)

\begin{tabular}{|l|l|}
\hline \multicolumn{2}{|c|}{ Specifications } \\
\hline Dimensions: & Temperature: \\
$83 \mathrm{~mm}$ x $42 \mathrm{~mm}$ x $11 \mathrm{~mm}$ & Standard: $-20^{\circ} \mathrm{C}$ to $60^{\circ} \mathrm{C}\left(-4^{\circ} \mathrm{F}\right.$ to $\left.140^{\circ} \mathrm{F}\right)$ \\
$\left(3.27^{\prime}\right.$ x $1.65^{\prime}$ x $\left.0.43^{\prime}\right)$ & Extended: $-30^{\circ} \mathrm{C}$ to $70^{\circ} \mathrm{C}\left(-22^{\circ} \mathrm{F}\right.$ to $\left.158^{\circ} \mathrm{F}\right)$ \\
\hline Weight: & Humidity: \\
$32 \mathrm{~g}(1.1 \mathrm{oz})$. & 0 to $95 \%$, non-condensing \\
\hline Enclosure: & Update rate: \\
Standard: IP63 & $0.00225 \mathrm{~Hz}$ up to 33.75Hz (can be varied dynamically \\
Extended: IP65 & under software control) \\
ABS (V0), UV Stabilized & \\
\hline Peripherals: & Radio frequencies: \\
LEDs (application controllable) & Ultra-wideband channel: \\
Buzzer (application controllable) & 6 - 8GHz \\
Two push buttons (application controllable) & Telemetry channel: \\
Motion detector & Narrow-band 2.4GHz \\
\hline Power supply: & Mounting options: \\
3V coin cell (CR2477) & Badge clip (supplied), cord lanyard \\
\hline Certifications: & Ubisense part codes: \\
US: FCC Part 15 & UBITAG7021 (Standard) \\
FCC IDs SEATAG21, SEATAG21HH & UBITAG7021X65 (Extended) \\
EU: CE & UBITAG7024 (Standard, handheld) \\
Canada: RSS-Gen, RSS-102, RSS-210, RSS- & UBITAG7024X65 (Extended, handheld) \\
220 & \\
Industry Canada ID 8673A-TAG21HH & \\
\hline
\end{tabular}




\section{Appendix B- ZigBee HA Wireless Metering Smart Plug Specifications}

The ZBMPlug15 enables remote control and monitoring of appliances by making them a part of a home automation network which is shown in Figure B.1. The ZBMPlug15smart plug also measures the delivered power. This enables the intelligent management of lighting and other appliances to take advantage of the lowest electric utilities rates while maximizing energy conservation. Use of the ZigBee home automation profile certifies compatibility with the emerging standards for smart homes/buildings (Smartenit, 2013).

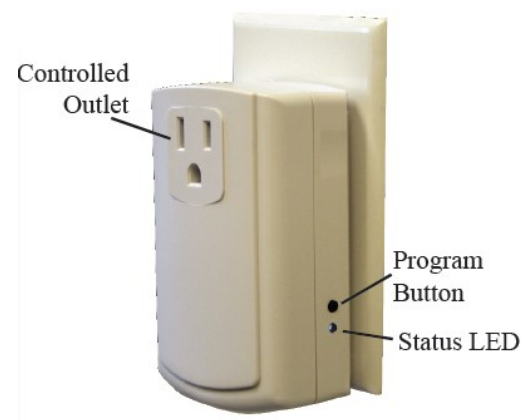

Figure B.1 Smart plug components (Smartenit, 2013)

\section{Installation}

The ZBMPlug15 must be installed in indoor environments or in a weather proof enclosure. Followings are the installation steps.

1) Reset to factory defaults

a) Plug ZBMPlug 15 into a $120 \mathrm{~V}$ AC receptacle (240V AC input is acceptable for North American receptacles).

b) Press and hold Program Button for 2 seconds. Release button after the LED goes out. LED will begin blinking rapidly.

c) Do not exceed $450 \mathrm{~W}$ of incandescent lighting or $15 \mathrm{~A}$ of resistive load 
2) Plug desired lamp or appliance into the Controlled Outlet (Smartenit, 2013).

\section{Set-up}

1) Joining the ZBMPlug15 to the Network

a) Open a ZigBee home automation router or coordinator to permit joining of theZBMPlug15 to the network.

b) When the ZBMPlug15 begins to join, the Status LED blinking rate will slow until it finally stops when successfully joined.

c) If the device continues to blink rapidly, ensure that the ZigBee home automation router or coordinator is still open for join and position the ZBMPlug15 as close as possible to the router or coordinator to eliminate the possibility for any range issues.

2) Binding the ZBMPlug15 to other devices (device must already be joined to network)

a) To bind the ZBMPlug15 to a ZigBee home automation controller, tap the program button once quickly. Then hold for 3 seconds and release. The Status LED will blink one short blip. Then press the program button on the controller device, such as a switch.

b) The duration of binding is determined by the coordinator (Smartenit, 2013).

\section{Open Permit Join}

The ZBMPlug15 serves as a router and allows other ZigBee home automation devices to join the network in addition to extending the range of ZigBee home automation devices. Following steps should be proceeding after the ZBMPlug15 has already joined the network i.e., LED is solid:

1) Press and hold the Program Button for 6 seconds. The Status LED will begin to flash once per second. The ZBMPlug15 will allow joining of other devices for up to 4 minutes.

2) Join the new ZigBee home automation device to the network according to the join method of that device. 
3) When the ZBMPlug15 has timed out for join permission, the status LED will stop flashing.

4) Permit join can be closed prior to time out by pressing the program button for 3 seconds (Smartenit, 2013).

\section{Rejoin}

The ZBMPlug15device will rejoin the network automatically if it loses power or needs to be moved to a new location once power has been restored. Until the device re-establishes communication with the network, the status LED will flash fast after which the LED will be lit solid (Smartenit, 2013).

\section{Manual Control}

Manual control is done through tapping the program button once. Tapping the program button toggles the load between ON and OFF. The status LED will is green when the load is ON and red when the load is OFF. The details of program button additional operation and status LED indications are explained in Tables B.1 and B.2, respectively (Smartenit, 2013).

Table B.1 Smart plug specifications (Smartenit, 2013)

\begin{tabular}{|l|l|}
\hline Operating Voltage & $95-240$ V AC \\
\hline Max. Load Current & $\begin{array}{l}\text { 15 Amps @ 120VAC } \\
\text { (10 Amps @ 240 VAC) }\end{array}$ \\
\hline Operating power & $\begin{array}{l}\text { Load energized: < 0.50 } \\
\text { Watts. } \\
\text { Standby: < 0.2 Watts. }\end{array}$ \\
\hline Surge protection & MOV rated for 300V \\
\hline Size & 4.0 ” L X 2.5” W X 1.5” H \\
\hline Weight & 4.1 Oz. \\
\hline Mounting & Through 3-pron \\
\hline Temperature & $25-50$ C \\
\hline Humidity & $\begin{array}{l}5-90 \% \text { RH (non- } \\
\text { condensing) }\end{array}$ \\
\hline
\end{tabular}


Table B.2 Status LED indication (Smartenit, 2013)

\begin{tabular}{|l|l|}
\hline \multicolumn{2}{|c|}{ Status LED Indication } \\
\hline Green LED & \multicolumn{1}{c|}{ Indication } \\
\hline Red LED Pattern & Load is ON \\
\hline \multicolumn{1}{|c|}{ Load is OFF } \\
\hline Fast flashing & \multicolumn{1}{c|}{ Indication } \\
\hline Solid & Searching for open network \\
\hline Short blip & Joined to network \\
\hline Flash once per second & Initiate binding to a controller \\
\hline Two blips per second & Open Permit Join \\
\hline & $\begin{array}{l}\text { Device cannot find "Parent } \\
\text { Device" and/or network it was } \\
\text { joined to }\end{array}$ \\
\hline
\end{tabular}




\section{Appendix C- Energy Simulation Configurations}

The specifications of Concordia University EV building which are used in the energy model simulation are explained below.

1) Floor and Ceiling: $225 \mathrm{~mm}$ reinforced concrete with electro conductive carpet tiles and mineral fiber suspended ceiling (type 3, shape 2, ASTM E-1264) with aluminum track system.

2) Indoor partition walls: Painted 3/8" gypsum board with metal studs, 3 " stud space filled with acoustic rock wool.

3) Door: Varnished $45 \mathrm{~mm}$ thick wood

The snapshots of the office model, cooling system, and heating system configurations are presented in Figures C.1, C.2, and C.3, respectively.



Figure C.1 Selected profile for the office 


\begin{tabular}{|c|c|c|c|c|}
\hline \multicolumn{2}{|c|}{ Apache Systems } & \multicolumn{2}{|r|}{$\Leftrightarrow$} & \begin{tabular}{|l|l|l|}
0 & $\square$ & $x$ \\
\end{tabular} \\
\hline \multirow{13}{*}{$\begin{array}{c}\text { Default? } \\
\checkmark \checkmark \\
\\
\checkmark\end{array}$} & $\begin{array}{l}\text { System Name } \\
\text { CentralHeatingRadiators }\end{array}$ & \multirow{2}{*}{$\begin{array}{r}\text { Name: } \\
\text { UK NCM type: }\end{array}$} & \multicolumn{2}{|c|}{ CentralHeatingRadiators } \\
\hline & $\begin{array}{l}\text { CentralHeatingRadiators } \\
\text { ActiveChilledBeams }\end{array}$ & & Central heating using water: radiators & \multirow[t]{2}{*}{ UK NCM wizard } \\
\hline & \multirow{11}{*}{ 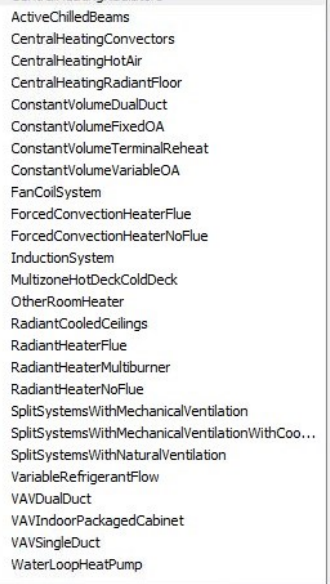 } & \multirow{7}{*}{$\begin{array}{l}\text { Heating Cooling Hot water } \\
\text { Generator: }\end{array}$} & \begin{tabular}{|l|l|l|l|l|} 
Solar water htg & Aux energy & Air supply & Cost & Control \\
\end{tabular} & \\
\hline & & & $\begin{array}{l}\text { Fuel Electricity } \\
\end{array}$ & \\
\hline & & & Is it a heat pump*? & $\square$ \\
\hline & & & Seasonal efficiency & 0.8900 \\
\hline & & & Delivery efficiency & 0.9393 \\
\hline & & & SCOP kW/kW & 0.8360 \\
\hline & & & Generator size $\mathrm{kW}$ & 0.00 \\
\hline & & \multirow[t]{2}{*}{ Heat recovery: } & Vent. heat recovery effectiveness & 0.0000 \\
\hline & & & Vent, heat recovery return air temp ${ }^{\circ} \mathrm{C}$ & 21.00 \\
\hline & & \multirow[t]{2}{*}{$\mathrm{CH}(\mathrm{C}) \mathrm{P}:$} & \multirow{2}{*}{$\begin{array}{l}\text { Is this heat source used in conjunction with CHP? } \\
\text { What ranking does this heat source have after the } \mathrm{CH}(\mathrm{C}) \mathrm{P} \text { plant? }\end{array}$} & 回 \\
\hline & & & & 1 \\
\hline Add & Remove & \multicolumn{2}{|l|}{ *-Applies to UK NCM only } & Cancel \\
\hline
\end{tabular}

\section{Figure C.2 Heating system configuration}

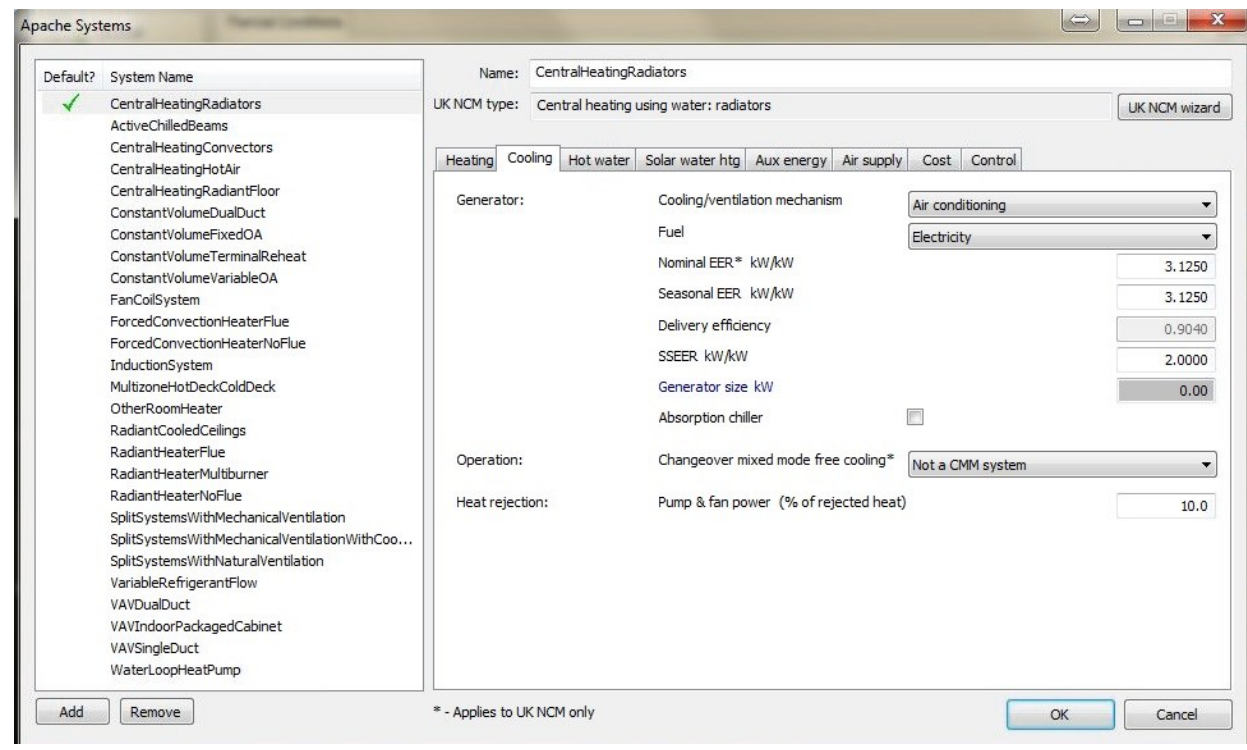

Figure C.3 Cooling system configuration 


\section{Appendix D- Smartplug Data Logging}

The instructions for proper use of the program and Smartplug Data Logging program are described for Smartplug Data Logging. The Python Program is developed by the Smartenit Company.

1) Create a folder where you would like the program to store the Data Logs.

2) Move the python program file in the folder created in step 1.

3) Run the python program.

4) The Program will create a CSV formatted log file for every Smartplug found in the Network.

5) Do not open the .CSV log files while the program is running. This can cause the program to crash.

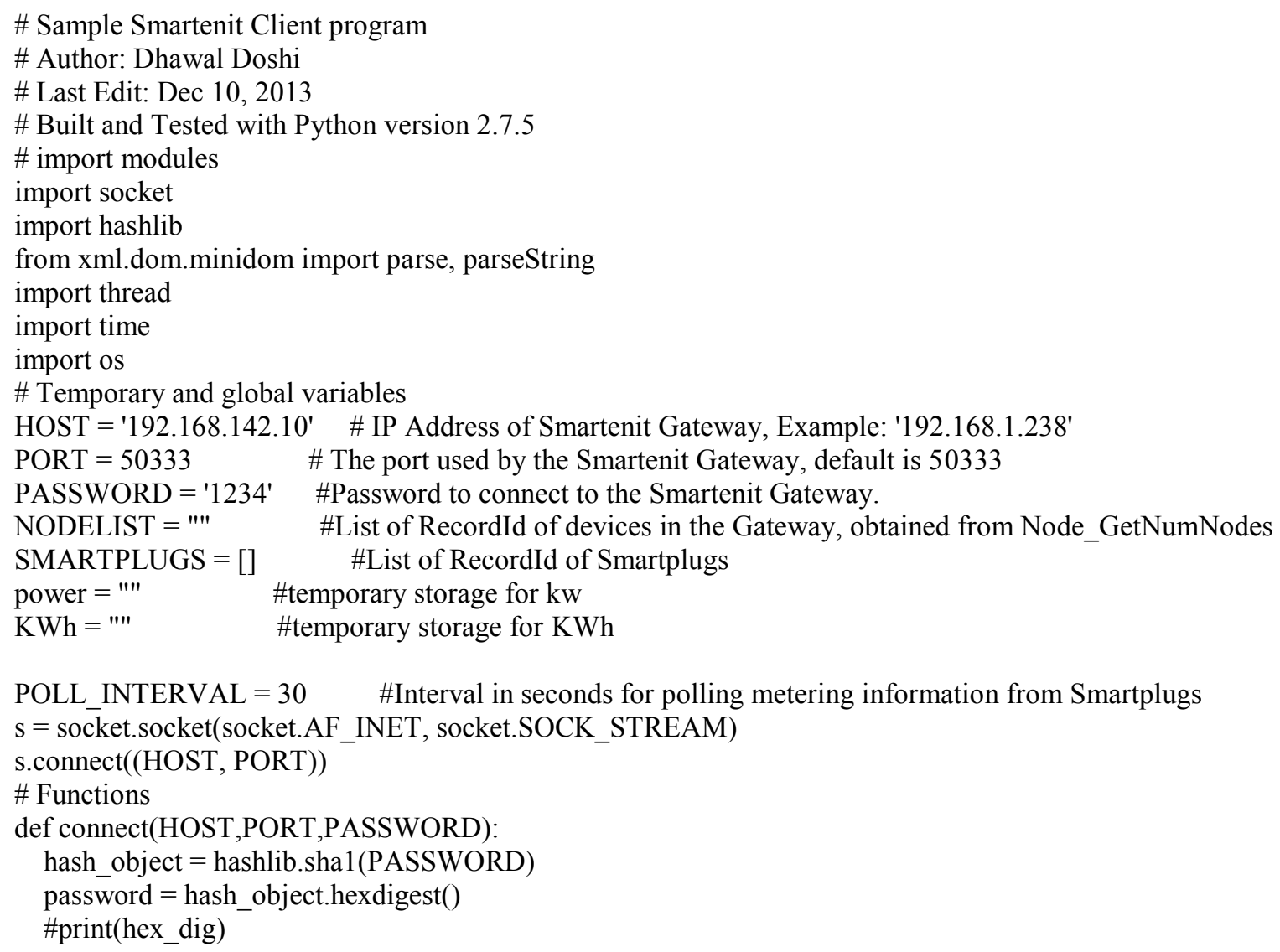


s.send(' $<$ zbpPacket $><$ Object $>$ ZBP_System $</$ Object $><$ methodName $>$ Sys_Authenticate $</$ methodName $><$ Arguments $><$ Argument type $=$ "string" $>$ '+ password $+{ }^{\prime}</$ Argument $></$ Arguments $><$ id $>12625</$ id $></$ zbpPacket $\left.>\backslash 0^{\prime}\right)$

def getnumnodes():

s.send(' $<$ zbpPacket $><$ Object $>$ ZBP_Node $</$ Object $><$ methodName $>$ Node_GetNumNodes $<$ methodName $><$ Argume nts $></$ Arguments $></$ zbpPacket $\left.>\mid 0^{\prime}\right)$

def Node GetNode(NODEID):

\#node $=$ int(NODEID,16)

NODEID $=\operatorname{str}(\operatorname{int}(\mathrm{NODEID}, 16))$

pkt

' $<$ zbpPacket $><$ Object $>$ ZBP_Node $<$ /Object $><$ methodName $>$ Node_GetNode $<$ /methodName $><$ Arguments $><$ Argume nt type="ushort" base="10" $>$ '+NODEID+'</Argument $></$ Arguments $><$ id $>1</$ id $></$ zbpPacket $>\mid 0^{\prime}$ \#pkt

' $<$ zbpPacket $><$ Object $>$ ZBP_Node $</$ Object $><$ methodName $>$ Node_GetNode $</$ methodName $><$ Arguments $><$ Argume nt type $=$ "ushort" $>$ '+NODEID $+{ }^{\prime}</$ Argument $></$ Arguments $></$ zbpPacket $>\mid 0^{\prime}$

\#print ' $\backslash \mathrm{r} \backslash \mathrm{n}$ ',pkt

s.send(pkt)

def Node_ClusterCommand(deviceid):

$\overline{\#} \mathrm{x}=\operatorname{int}($ deviceid, 16$)$

\#nodeid_dec $=\operatorname{str}(\mathrm{x})$

s.send('<zbpPacket $><$ Object $>$ ZBP_Node $</$ Object $><$ methodName $>$ Node_ClusterCommand $<$ /methodName

$><$ Arguments $><$ Argument type="uchar" base="10" $>146</$ Argument $><$ Argument type $=$ "ushort"

base $=" 10 ">$ ' + str(deviceid) + ' $</$ Argument $><$ Argument $\quad$ type $=$ "uchar" $\quad$ base $=" 10 ">2</$ Argument $><$ Argument

type="ushort" base="10" $>1794</$ Argument $><$ Argument type $=$ "uchar" base $=" 10$ " $>0</$ Argument $><$ Argument type $="$ QByteArray" base $=" 10 ">[2,0,0,4,0]</$ Argument $></$ Arguments $><$ id $>7</$ id $></$ zbpPacket $\left.>\mid 0^{\prime}\right)$

def getnode_details():

while True:

nodes $=$ len(NODELIST)

if(nodes $>0$ ):

\#print 'Inside getnode_details'

\#print("NodeList: "),NODELIST

\#print("\n")

for $\mathrm{x}$ in NODELIST:

\#print 'InGetting Node: ', $\mathrm{x}$

Node_GetNode(x)

time. $\operatorname{sleep}(0.1)$

break

def poll_smartplugs():

time.sleep $(10)$

while True:

\#print 'Inside poll_smartplugs'

nodes $=$ len(SMARTPLUGS)

\#print SMARTPLUGS

\#print("\n")

\#print len(SMARTPLUGS)

if(nodes $>0)$ :

\#print 'Inside poll_smartplugs'

\#print("Smartplugs: "),SMARTPLUGS

\#print("\n")

for deviceid in SMARTPLUGS:

print 'Getting Metering Information from: '+str(deviceid)+' $\backslash n$ '

Node_ClusterCommand(deviceid)

time.sleep (5)

\#break

time.sleep(POLL_INTERVAL) 


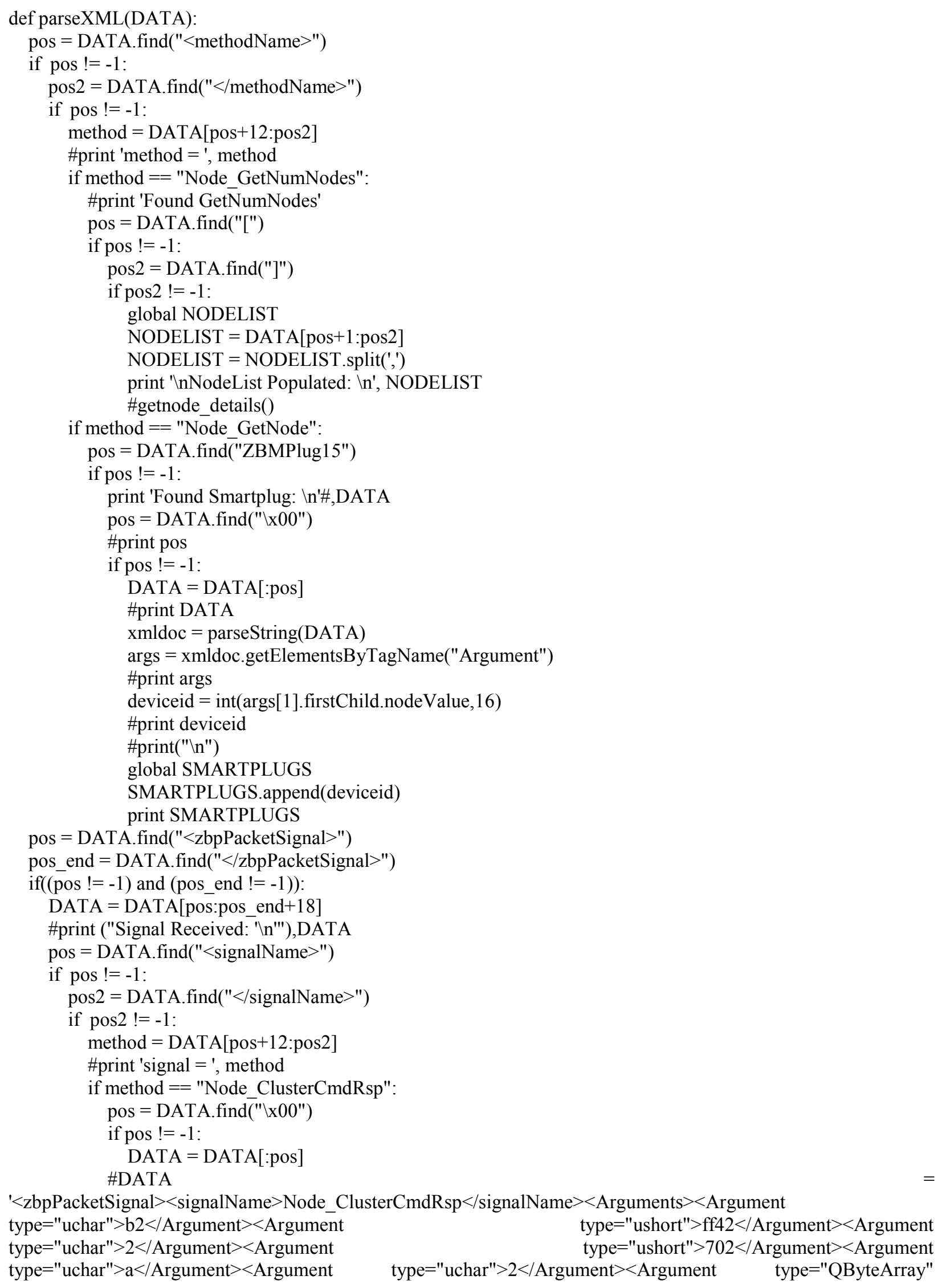


length $=" 15 ">[4,0,2 \mathrm{a}, 0,0,0,0,0,25,0,0,0,0,46,2 \mathrm{~b}]</$ Argument $><$ Argument

type $=$ "uchar" $>2</$ Argument $></$ Arguments $></$ zbpPacketSignal $>$ '

DATA $=\operatorname{str}($ DATA)

\#print ("Metering Info Received: '\n"'),DATA

xmldoc $=$ parseString $($ DATA $)$

args = xmldoc.getElementsByTagName("Argument")

deviceid = int(args[1].firstChild.nodeValue,16)

\#print ("Device ID: "), deviceid

commandid $=\operatorname{int}(\operatorname{str}(\operatorname{args}[4]$. firstChild.nodeValue $), 16$ )

clusterid $=\operatorname{int}(\operatorname{str}(\operatorname{args}[3]$.firstChild.nodeValue),16)

meterdata $=\operatorname{str}(\operatorname{args}[6]$. firstChild.nodeValue)

meterdata $=$ meterdata[1:-1] \#removing []

meterdata $=$ meterdata.split(',')

\#rawmeter $=$ meterdata

\#print ("Received Raw Data: \%s $\backslash n$ ") \% rawmeter

meterdata $=[\operatorname{int}(i, 16)$ for $i$ in meterdata $]$

\#proc meterdata $=$ meterdata

if $($ commandid $==1)$ :

print "Processing synch response $\backslash r$ "

\#print "Processing synchronous response: \%s $\backslash n$ " \% proc_meterdata

if $($ clusterid $==1794)$ :

if $($ len (meterdata) $>=9)$ :

for $i$ in range(len(meterdata)):

$\operatorname{if}(($ len(meterdata $))-9)>=\mathrm{i})$

if $((\operatorname{meterdata}[\mathrm{i}]=0) \operatorname{and}(\operatorname{meterdata}[\mathrm{i}+1]==0) \operatorname{and}(\operatorname{meterdata}[\mathrm{i}+2] \quad==$

$0)$ and $(\operatorname{meterdata}[i+3]==37))$ :

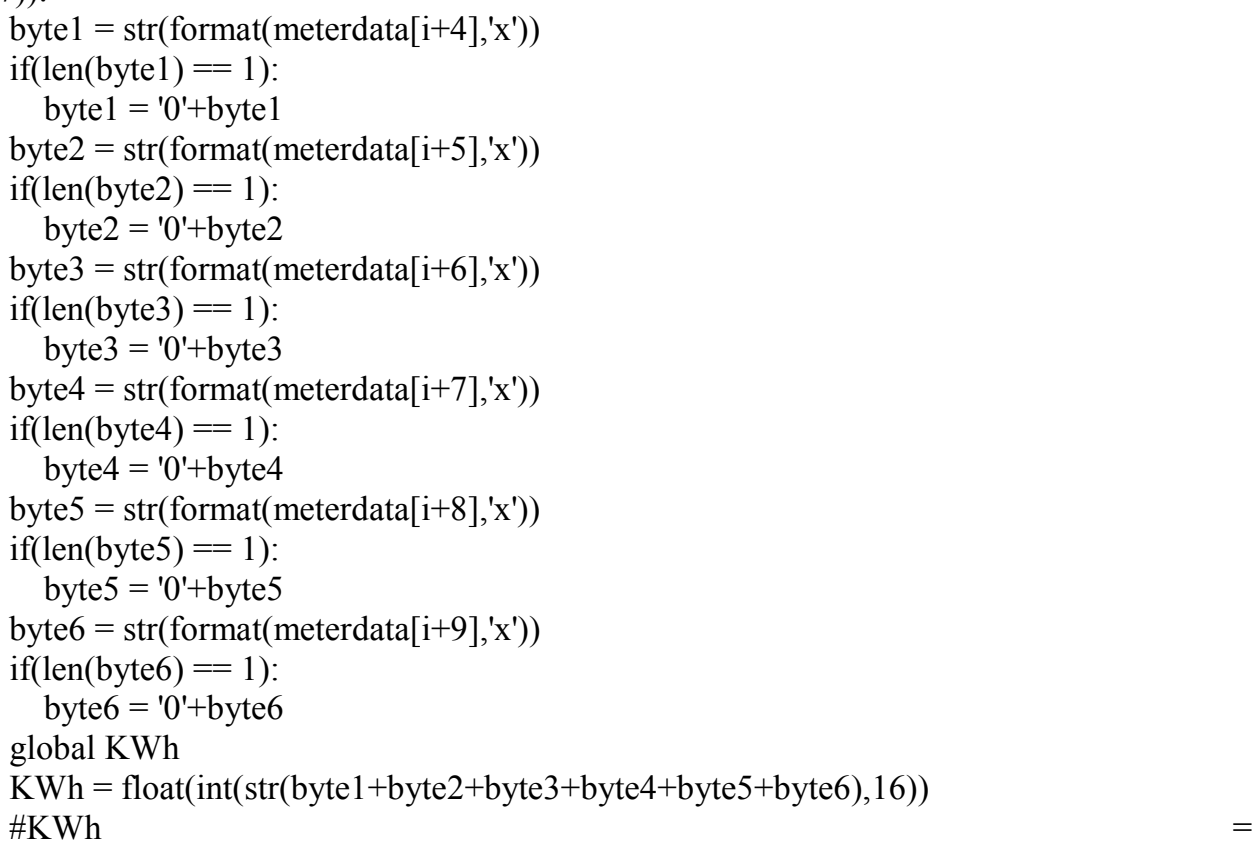

float(int $\left(\operatorname{str}\left(\right.\right.$ format $\left(\right.$ meterdata[ $\left.\left.[i+4], x^{\prime}\right)\right)+\operatorname{str}\left(\right.$ format $\left(\right.$ meterdata $\left.\left.[i+5], x^{\prime}\right)\right)+\operatorname{str}\left(\right.$ format $\left.\left(\operatorname{meterdata}[i+6],{ }^{\prime}\right)\right)+\operatorname{str}($ format $(m$ eterdata[i+7],'x'))+str(format (meterdata[i+8],'x')) $+\operatorname{str}\left(\right.$ format $\left(\right.$ meterdata $\left.\left.\left.[i+9],{ }^{\prime}{ }^{\prime}\right)\right), 16\right)$ )

$\mathrm{KWh}=\mathrm{KWh} / 100000$

break

if(len(meterdata) $>=6)$ :

for $\mathrm{k}$ in range(len(meterdata)):

if $((($ len(meterdata $))-6)>=\mathrm{i})$ :

$0)$ and $($ meterdata $[\mathrm{k}+3]==42))$ :

if $((\operatorname{meterdata}[\mathrm{k}]==4) \operatorname{and}(\operatorname{meterdata}[\mathrm{k}+1]==\quad 0) \operatorname{and}(\operatorname{meterdata}[\mathrm{k}+2]==$ 


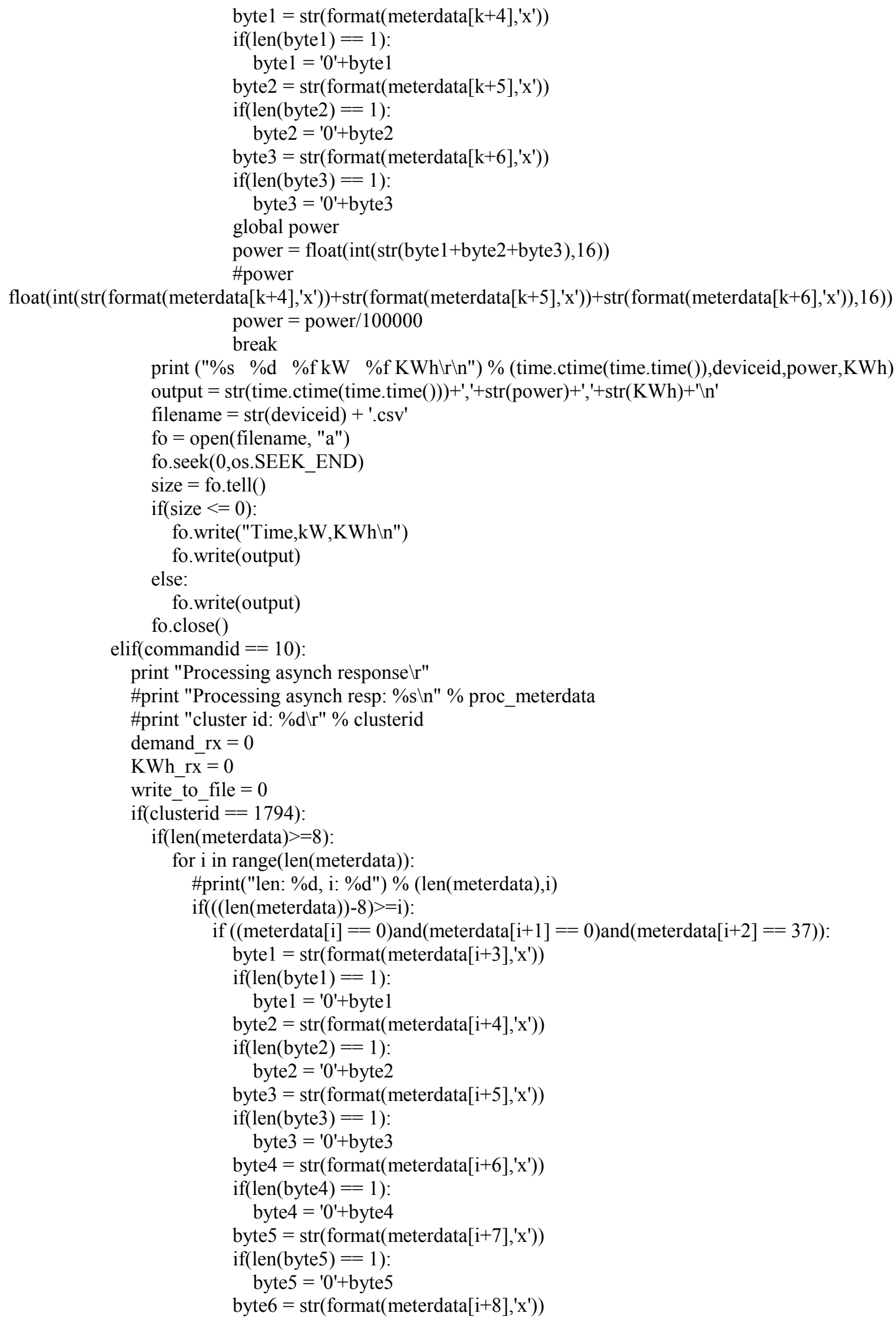


if $($ len $($ byte 6$)==1)$ :
byte $6=$ '0'+byte 6

global KWh

$\mathrm{KWh}=$ float(int(str(byte1+byte2+byte3+byte4+byte5+byte6),16))

$\# \mathrm{KWh}$

float $\left(\operatorname{int}\left(\operatorname{str}\left(\right.\right.\right.$ format $\left(\right.$ meterdata[ $\left.\left.[i+3],{ }^{\prime} x^{\prime}\right)\right)+\operatorname{str}\left(\right.$ format $\left(\right.$ meterdata[ $\left.\left.[i+4], x^{\prime}\right)\right)+\operatorname{str}\left(\right.$ format $\left(\right.$ meterdata $\left.\left.[i+5],{ }^{\prime} x^{\prime}\right)\right)+\operatorname{str}($ format $(m$ eterdata[i+6],'x') $)+\operatorname{str}\left(\right.$ format $\left(\right.$ meterdata[i+7],' $\left.\left.x^{\prime}\right)\right)+\operatorname{str}\left(\right.$ format $\left(\right.$ meterdata[ $\left.\left.\left.\left.[i+8], x^{\prime}\right)\right), 16\right)\right)$

$\mathrm{KWh}=\mathrm{KWh} / 100000$

$\mathrm{KWh} \mathrm{rx}=1$

break

if(len(meterdata) $>=5)$ :

for $\mathrm{k}$ in range(len(meterdata)):

\#print("len: \%d, k: \%d") \% (len(meterdata),k)

if $(($ len (meterdata) $)-5)>=\mathrm{k})$ :

if $(($ meterdata $[\mathrm{k}]==4)$ and $(\operatorname{meterdata}[\mathrm{k}+1]==0) \operatorname{and}(\operatorname{meterdata}[\mathrm{k}+2]==42))$ :

byte $1=\operatorname{str}\left(\right.$ format $\left(\right.$ meterdata $\left.\left.[\mathrm{k}+3], \mathrm{x}^{\prime}\right)\right)$

if $($ len $($ byte 1$)==1)$ :

byte $1=$ ' 0 '+byte 1

byte $2=\operatorname{str}\left(\right.$ format $\left(\right.$ meterdata $\left.\left.[\mathrm{k}+4], \mathrm{x}^{\prime}\right)\right)$

if $($ len $($ byte 2$)==1)$ :

byte $2=$ ' 0 ' + byte 2

byte $3=\operatorname{str}\left(\right.$ format $($ meterdata[k +5$\left.\left.], x^{\prime}\right)\right)$

if $($ len $($ byte 3$)==1)$ :

byte $3={ }^{\prime} 0$ '+byte 3

global power

power $=$ float $(\operatorname{int}(\operatorname{str}($ byte $1+$ byte $2+$ byte 3$), 16))$

\#power

float $\left(\operatorname{int}\left(\operatorname{str}\left(\right.\right.\right.$ format $\left(\right.$ meterdata[k+3],' $\left.\left.x^{\prime}\right)\right)+\operatorname{str}\left(\right.$ format $($ meterdata[k+4],'x') $)+\operatorname{str}\left(\right.$ format $\left(\right.$ meterdata[k+5],' $\left.\left.\left.\left.x^{\prime}\right)\right), 16\right)\right)$

power $=$ power $/ 100000$

demand_rx $=1$

break

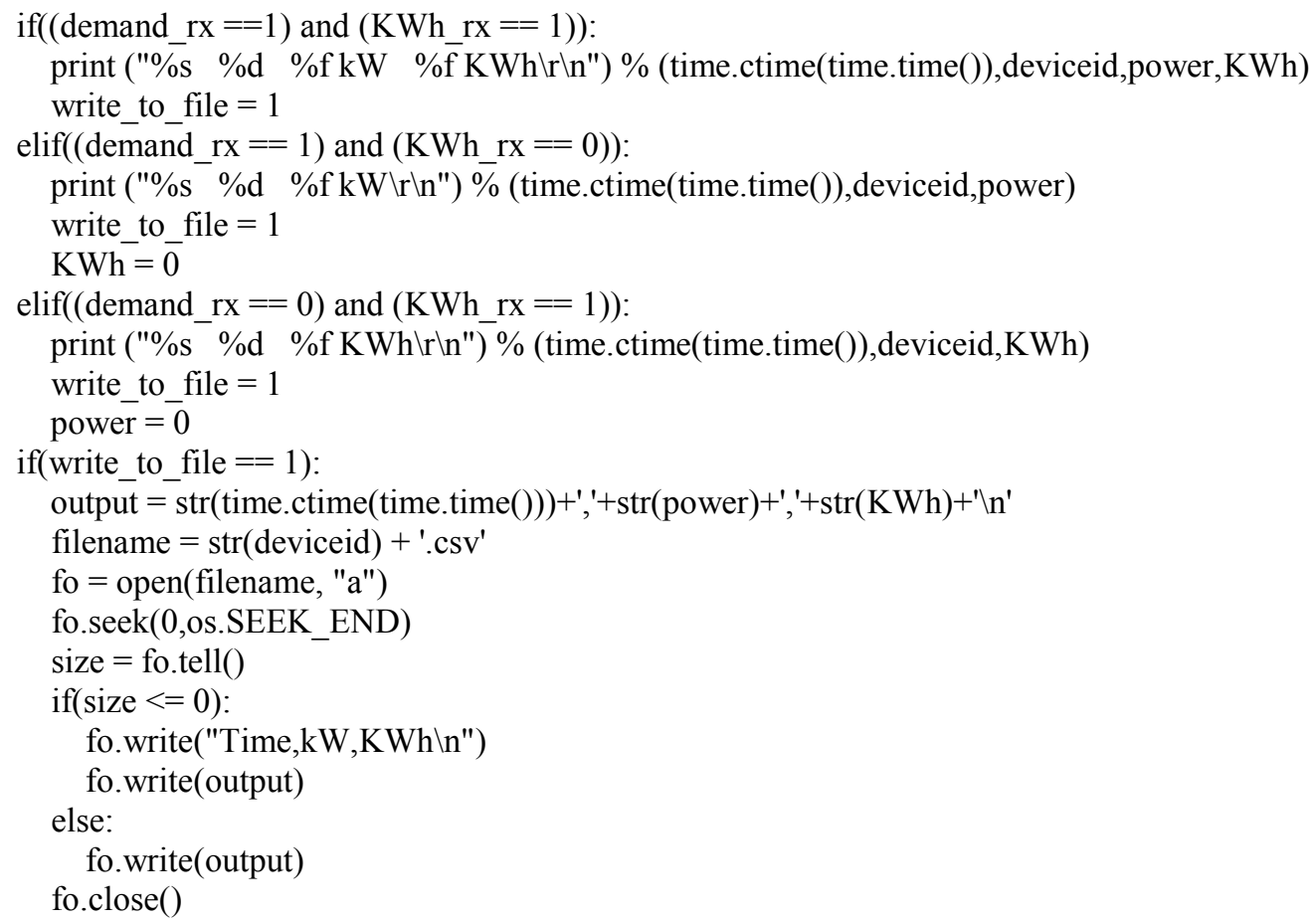




$$
\begin{aligned}
& \text { write_to_file }=0 \\
& \text { demand_rx }=0 \\
& \text { KWh_rx }=0
\end{aligned}
$$

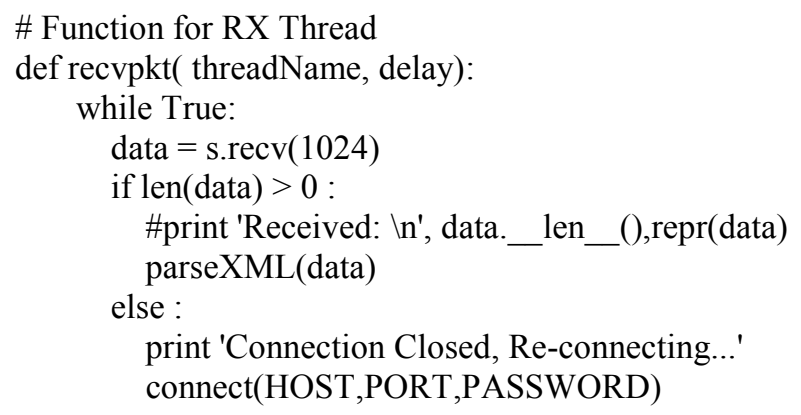

